\title{
Foodstuff Concentrations following a SRTC Tritium Oxide Release
}

by

A. Blanchard

Westinghouse Savannah River Company

Savannah River Site

Aiken, South Carolina 29808

D. R. Marx

Westinghouse Safety Management Solutions

This paper was prepared in connection with work done under the above contract number with the U.S. Department of Energy. By acceptance of this paper, the publisher and/or recipient acknowledges the U. S. Government's right to retain a nonexclusive, royalty-free license in and to any copyright covering this paper, along with the right to reproduce and to authorize others to reproduce all or part of the copyrighted paper. 


\section{Foodstuff Concentrations Following a SRTC Tritium Oxide Release (U)}

D.R. Marx

April 1998

Classification

UNCLASSIFIED

DOES NOT CONTAIN

UNCLASSIFIED CONTROLLED

NUCLEAR INFORMATION
ADC \&

Reviewing

Official:

K.R. O'Kula, Managing Member, Consequence Analysis Date:
Westinghouse Savannah River Company

Aiken, SC 29808

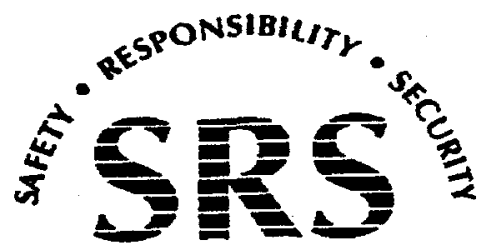

SAVANNAH RIVER SITE

PREPARED FOR THE U.S. DEPARTMENT OF ENERGY UNDER CONTRACT NO. DE-AC09-96SR18500 
Keywords:

Ingestion Pathways

Tritium Oxide Release

Foodstuff Concentrations

Consequence Analysis

\section{Foodstuff Concentrations following a SRTC Tritium Oxide Release (U)}

D.R. Marx

April 1999

Westinghouse Savannah River Company Aiken, SC 29808

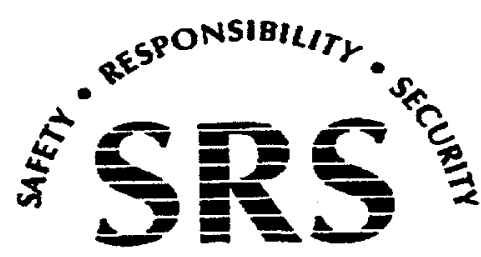

SAVANNAH RIVER SITE

PREPARED FOR THE U.S. DEPARTMENT OF ENERGY UNDER CONTRACT NO. DE-AC09-96SR18500 


\section{DISCLAIMER}

This report was prepared as an account of work sponsored by an agency of the United States Government. Neither the United States Government nor any agency thereof, nor any of their employees, makes any warranty, express or implied, or assumes any legal liability or responsibility for the accuracy, completeness, or usefulness of any information, apparatus, product, or process disclosed, or represents that its use would not infringe privately owned rights. Reference herein to any specific commercial product, process, or service by trade name, trademark, manufacturer, or otherwise does not necessarily constitute or imply its endorsement, recommendation, or favoring by the United States Government or any agency thereof. The views and opinions of authors expressed herein do not necessarily state or reflect those of the United States Government or any agency thereof.

This report has been reproduced directly from the best available copy.

Available to DOE and DOE contractors from the Office of Scientific and Technical Information, P.O. Box 62, Oak Ridge, TN 37831; prices available from (615) 576-8401.

Available to the public from the National Technical Information Service, U.S. Department of Commerce, 5285 Port Royal Road, Springfield, VA 22161. 


\section{DISCLAIMER}

Portions of this document may be illegible in electronic image products. Images are produced from the best available original document. 
Project: Ingestion Planning Zone Emergency Preparedness Issues

Document: WSRC-TR-99-00117

Title:

Foodstuff Concentrations Following a SRTC Tritium Oxide Release (U)

WSMS Approvals:

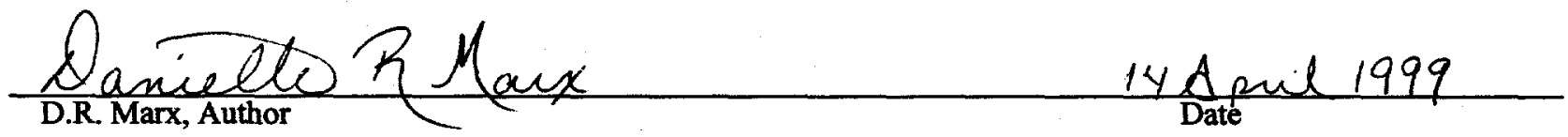

$\frac{1 m \sigma}{\text { f.M. Thompson, 7echnical Reviewer }} \quad 4 / 14 / 99$
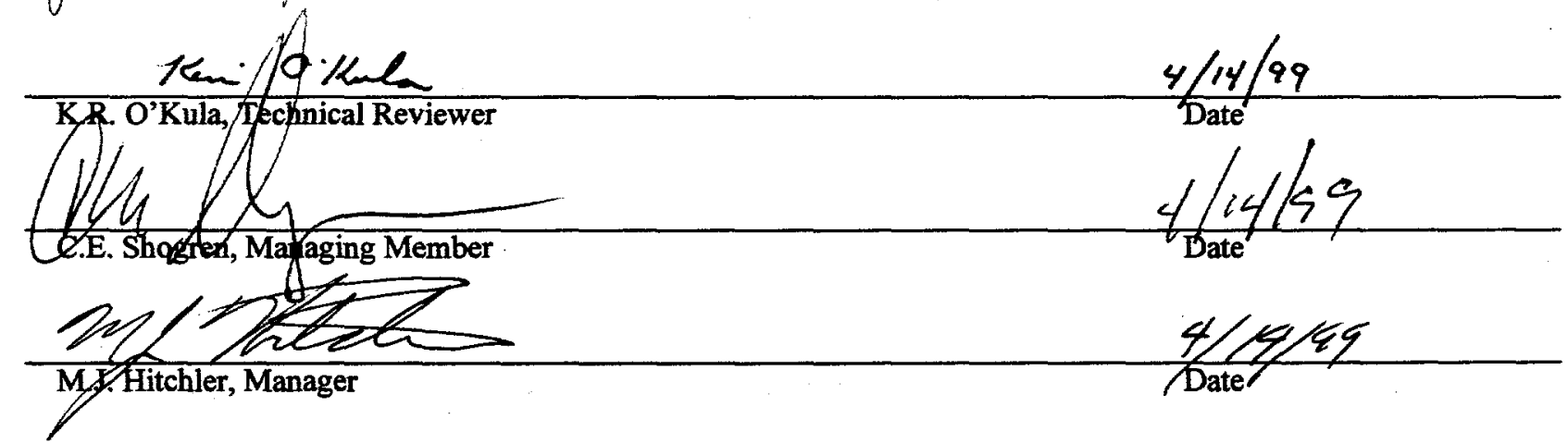
This Page Intentionally Left Blank 


\section{EXECUTIVE SUMMARY}

The ingestion pathway consequences following a postulated accidental tritium release from the Savannah River Technology Center are evaluated. The tritium release, part of scenario 1-RD-3, a full facility fire in Building 773-A, was examined for both average and adverse meteorological conditions. Tritium concentrations in vegetables, wheat grain, meat, and milk were calculated using the tritium dispersion and dose assessment model UFOTRI. Under average meteorological conditions, the peak HTO concentrations for all foodstuffs at $1 \mathrm{~km}$ and vegetables at $10 \mathrm{~km}$ exceed the DIL. At one week, all foodstuffs at $1 \mathrm{~km}$ exceed the DIL. After one year, the only foodstuff to exceed the DIL is wheat grain at $0.67 \mathrm{~km}$. For adverse meteorological conditions, the peak HTO concentrations for all foodstuffs at $1 \mathrm{~km}$, wheat grain at $10 \mathrm{~km}$, and vegetables at $50 \mathrm{~km}$, exceed the DIL. At one week, all categories at $1 \mathrm{~km}$ and milk at $10 \mathrm{~km}$ exceed the DIL. After one year, wheat grain concentrations continue to exceed the DIL at $1 \mathrm{~km}$. 
This Page Intentionally Left Blank 


\section{Table of Contents}

1.0 INTRODUCTION......................................................................................... 1

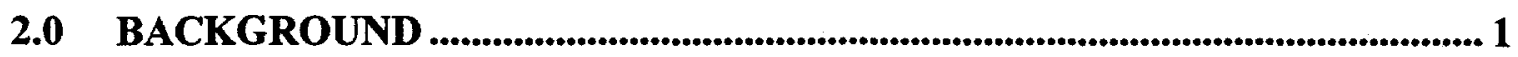

2.1 Scenario Examined ...................................................................................... 1

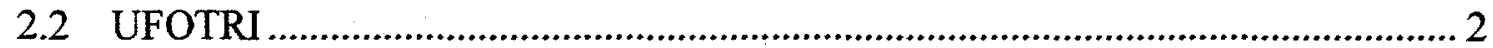

3.0 FDA GUIDANCE AND EVALUATION CRITERIA....................................... 2

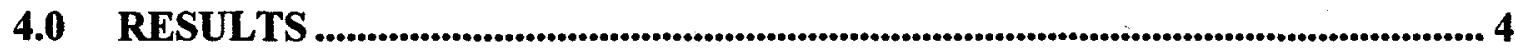

5.0 CONCLUSIONS

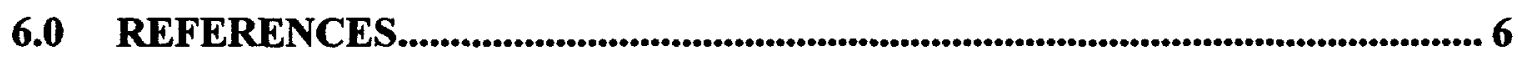

APPENDIX A UFOTRI INPUT DECK FOR SRTC RELEASE - AVERAGE MET CONDITIONS......................................................................................................... 7 APPENDIX B RADIONUCLIDE CONCENTRATIONS IN FOODSTUFFS .......... 9 


\section{List of Tables}

Table 1. Tritium releases for SRTC scenario 1-RD-3, without and with DR applied...... 1

Table 2. Meteorological conditions for SRTC tritium release..........................2

Table 3. Additional Input Parameters for UFOTRI...................................2

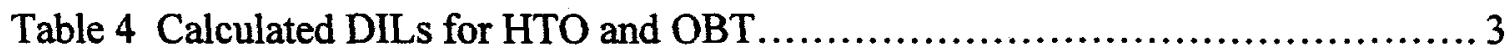

Table 5. Tritium Concentrations for Average Meteorological Conditions (Max HTO)...4

Table 6. Tritium Concentrations for Average Meteorological Conditions ( 1 week)......4

Table 7. Tritium Concentrations for Average Meteorological Conditions (1 year)....... 4

Table 8. Tritium Concentrations for Adverse Meteorological Conditions (Max HTO)...4

Table 9. Tritium Concentrations for Adverse Meteorological Conditions (1 week)........4

Table 10. Tritium Concentrations for Adverse Meteorological Conditions (1 year)..... 5 


\subsection{INTRODUCTION}

Accidental releases of radionuclides from nuclear facilities may cause contamination of food and forage. Numerous pathways exist for deposited radionuclides to reach man. The major pathways considered by recent Food and Drug Administration (FDA) guidance (FDA 1998) are analyzed in this report for a postulated accidental tritium release from the Savannah River Technology Center (SRTC).

\subsection{BACKGROUND}

The scenario examined and the tritium dispersion and dose assessment model UFOTRI are discussed below.

\subsection{Scenario Examined}

The release examined for this report is the tritium portion of SRTC release scenario 1-RD-3, a full facility fire in Building 773-A described in Attachments 2, 3, and 5 of S-EHA-A-00001, Rev. 1, Appendix B, Calculation 1 (Hadlock 1997). The particulate portion of the source term is examined in WSRC-TR-99-00090 (Thompson 1999a). The respirable source terms described in the SRTC EPHA are derived from a detailed analysis involving consideration of physical forms and disbursing mechanisms. An overall damage ratio (DR) is applied to the overall source term from the Attachments in S-EHA-A-00001, Rev. 1, Appendix B, Calculation 1 (see sheet 8 of 30 for the DR specification). In general, to determine the total release, it is necessary to infer the respirable fraction (RF) from the respirable release source terms. For tritium, the RF is 1.0 . The pre-DR source term is derived in S-CLC-A-00104 (Thompson 1999b). The final step is to apply a damage ratio (DR) of 0.75 , consistent with the DR assumed for respirable releases in the SRTC EPHA. These quantities are summarized in Table 1 for tritium.

Table 1. Tritium releases for SRTC scenario 1-RD-3, without and with DR applied

\begin{tabular}{ccc}
\hline Nuclide & $\begin{array}{c}\text { Pre-DR airborne release } \\
(\mathrm{Ci})\end{array}$ & $\begin{array}{c}\mathrm{DR}=0.75, \text { unmixed total release } \\
(\mathrm{Ci})\end{array}$ \\
\hline $\mathrm{H} 3$ & $2.14 \mathrm{E}+04$ & $1.61 \mathrm{E}+04$ \\
\hline
\end{tabular}

The tritium dispersion and dose assessment model UFOTRI was selected to evaluate the consequences for the tritium release (Section 2.2). Two meteorological conditions - average and 95\% adverse - are examined (Hadlock 1997). Table 2 lists the meteorological parameters for each condition. 
Table 2. Meteorological conditions for SRTC tritium release (Hadlock 1997)

\begin{tabular}{ccc}
\hline Parameter & Average Meteorology & $95 \%$ Adverse Meteorology \\
Release Height & Ground & Ground \\
Wind Speed & $2.5 \mathrm{~m} / \mathrm{s}$ & $1.7 \mathrm{~m} / \mathrm{s}$ \\
Surface Roughness & $100 \mathrm{~cm}$ & $100 \mathrm{~cm}$ \\
Stability class & $\mathrm{C}$ & $\mathrm{E}$ \\
Release duration & $60 \mathrm{~min}$ & $60 \mathrm{~min}$ \\
Inversion layer & $500 \mathrm{~m}$ & $200 \mathrm{~m}$ \\
\hline
\end{tabular}

\section{$2.2 \quad$ UFOTRI}

The tritium dispersion and dose assessment model UFOTRI (Unfallfolgenmodell für Tritiumfreisetzungen) has been chosen to model the SRTC tritium release. UFOTRI was developed at the German Karlsruhe laboratory to assess the radiological consequences of accidental atmospheric tritium releases (Raskob 1990, 1993). UFOTRI explicitly addresses all applicable biophysics of tritium dispersion, its interaction in the environment, and the ultimate uptake by downwind receptors. UFOTRI realistically models the atmospheric dispersion, resuspension, and downwind deposition of tritium. Additionally, UFOTRI couples the shortterm atmospheric dispersion model with a first order compartment module that dynamically describes the long-term behavior of tritium in the environment (Raskob 1990, 1993).

Table 3 provides a listing of some of the additional parameters required in UFOTRI (the input file for the adverse meteorological parameters is located in Appendix A).

Table 3. Additional Input Parameters for UFOTRI

\begin{tabular}{|c|c|c|c|}
\hline Additional Information & & Meteorology & \\
\hline $\begin{array}{l}\text { Chemical Forms } \\
\text { No building wake }\end{array}$ & $0 \%$ HT $/ 100 \%$ HTO & $\begin{array}{l}\text { Air temperature } \\
\text { No Rainfall }\end{array}$ & $\begin{array}{l}32.22^{\circ} \mathrm{C} \\
0 \mathrm{~mm} / \mathrm{h}\end{array}$ \\
\hline Initial soil water content & $35 \%$ & Thermal energy of release & $0 \mathrm{cal} / \mathrm{sec}$ \\
\hline Relative humidity & 0.8 & Wind direction (in degrees) & \\
\hline & & Dispersion parameters & Briggs \\
\hline $\begin{array}{l}\text { Soil and vegetation } \\
\text { Soil type } \\
\text { Pore volume (default) }\end{array}$ & $\begin{array}{l}\text { Sandy loam } \\
50 \%\end{array}$ & $\begin{array}{l}\text { Code coefficients } \\
\text { HTO dry deposition velocity* } \\
\text { HT dry deposition velocity* }\end{array}$ & $\begin{array}{l}0.5 \mathrm{~cm} / \mathrm{s} \\
0.05 \mathrm{~cm} / \mathrm{s}\end{array}$ \\
\hline
\end{tabular}

\subsection{FDA Guidance AND Evaluation CRiteria}

The Food and Drug Administration (FDA) Derived Intervention Levels (DILs) are limits on the concentrations permitted in human food distributed in commerce to prevent consumption of undesirable amounts of radionuclides and apply in the first year after an accident (FDA 1998). The DIL corresponds to the concentration in food present throughout the relevant period of time that, in the absence of any intervention, could lead to an individual receiving a radiation dose equal to the Protective Action Guide (PAG). The PAGs for the ingestion pathway are $0.5 \mathrm{rem}$ 
committed effective dose equivalent or 5 rem committed dose equivalent to an individual tissue or organ, whichever is most limiting.

FDA (1998) places selected radionuclides into five primary groups, each with common characteristics. These groups are:

- $\operatorname{Sr}-90$

- I-131

- $\mathrm{Cs}-134+\mathrm{Cs}-137$
- $\mathrm{Pu}-238+\mathrm{Pu}-239+\mathrm{Am}-241$

- $\mathrm{Ru}-103+\mathrm{Ru}-106$

The five radionuclide groups are not sufficient to cover accidents at all types of facilities or locations. Appendix E of FDA (1998) presents DILs for 15 additional radionuclides. DILs for tritiated water (HTO) and organically bound tritium (OBT) were derived using methodology consistent with FDA (1998) and are listed in Table 4 (Tharakan 1999).

Table 4 Calculated DILs for HTO and OBT

\begin{tabular}{|c|c|c|c|}
\hline $\begin{array}{c}\text { Radionuclide / } \\
\text { Group }\end{array}$ & DIL (Ci/g) & $\begin{array}{c}\text { Radionuclide } / \\
\text { Group }\end{array}$ & DIL (Ci/g) \\
\hline HTO & $5.9 \mathrm{E}-09$ & OBT & $2.4 \mathrm{E}-09$ \\
\hline
\end{tabular}

To evaluate the release, calculated concentrations in vegetables, wheat grain, meat, and milk for multiple distances and times following the release will be compared with the HTO and OBT DILs. Tritium concentrations in grass, although calculated in UFOTRI, were not examined since grass is not directly consumed by humans. However, tritium concentrations in grass are indirectly incorporated via the meat and milk pathways. In all instances, the HTO and OBT concentrations will be summed prior to comparison with the appropriate DIL.

For short-term concentrations, the time at which the peak HTO concentration occurs in each food type will be examined. The summed HTO and OBT concentrations will be compared with the HTO DIL. In the time immediately following the release, the HTO concentration quickly reaches its peak and is the dominant form of tritium present in foodstuffs.

For long-term concentrations, two evaluation times - one week (168 hours) and one year (8760 hours) - will be examined. The OBT DIL will be used for these comparisons because the tritium will be incorporated into carbon compounds that have a greater biological half-life than HTO. For plants, the HTO half-life is approximately one hour while the OBT half-life ranges from 8 hours to over 10 days. Examination of long-term concentrations is useful to determine if intervention is still required.

Concentrations for both the short and long-term will be examined at the site boundary $(0.67 \mathrm{~km})$, $1 \mathrm{~km}, 10 \mathrm{~km}, 50 \mathrm{~km}$, and $100 \mathrm{~km}$ from the release. The site boundary concentrations may be used to determine if any intervention will be necessary. The $100 \mathrm{~km}$ value is used for comparison with the particulate concentrations. (The code used to calculate concentrations for particulates, HOTSPOT, calculates up to a maximum distance of $100 \mathrm{~km}$.) The remaining distances are chosen for additional examination. 


\subsection{RESULTS}

Calculated tritium concentrations for average and adverse meteorological conditions are presented in Tables 5 to 7 and 8 to 10, respectively (summarized from Appendix B). Concentrations listed are the sum of the HTO and OBT concentrations. Values in bold surpass the appropriate DIL. Peak HTO concentrations are reached between 1 and 50 hours following the release, depending upon distance from the release, atmospheric conditions, and transport time.

Table 5. Tritium Concentrations for Average Meteorological Conditions (Max HTO)

\begin{tabular}{lcccccc}
\hline Pathway & DIL & $\begin{array}{c}0.67 \mathrm{~km} \\
\mathrm{Ci} / \mathrm{g}\end{array}$ & $\begin{array}{c}1 \mathrm{~km} \\
\mathrm{Ci} / \mathrm{g}\end{array}$ & $\begin{array}{c}10 \mathrm{~km} \\
\mathrm{Ci} / \mathrm{g}\end{array}$ & $\begin{array}{c}50 \mathrm{~km} \\
\mathrm{Ci} / \mathrm{g}\end{array}$ & $\begin{array}{c}100 \mathrm{~km} \\
\mathrm{Ci} / \mathrm{g}\end{array}$ \\
\hline Vegetable & $5.9 \mathrm{E}-09$ & $\mathbf{3 . 8 E}-07$ & $2.0 \mathrm{E}-07$ & $9.2 \mathrm{E}-09$ & $2.0 \mathrm{E}-09$ & $7.4 \mathrm{E}-10$ \\
Wheat grain & $5.9 \mathrm{E}-09$ & $\mathbf{2 . 1 E - 0 7}$ & $\mathbf{1 . 1 E - 0 7}$ & $5.6 \mathrm{E}-09$ & $1.5 \mathrm{E}-09$ & $7.0 \mathrm{E}-10$ \\
Meat & $5.9 \mathrm{E}-09$ & $\mathbf{1 . 8 E - 0 8}$ & $\mathbf{9 . 3 E}-09$ & $4.8 \mathrm{E}-10$ & $3.0 \mathrm{E}-11$ & $2.7 \mathrm{E}-11$ \\
Milk & $5.9 \mathrm{E}-09$ & $\mathbf{2 . 2 E - 0 8}$ & $\mathbf{1 . 2 E - 0 8}$ & $6.1 \mathrm{E}-10$ & $3.9 \mathrm{E}-11$ & $3.4 \mathrm{E}-11$ \\
\hline
\end{tabular}

Table 6. Tritium Concentrations for Average Meteorological Conditions (1 week)

\begin{tabular}{lcccccc}
\hline Pathway & DIL & $0.67 \mathrm{~km}$ & $1 \mathrm{~km}$ & $10 \mathrm{~km}$ & $50 \mathrm{~km}$ & $100 \mathrm{~km}$ \\
& $\mathrm{Ci} / \mathrm{g}$ & $\mathrm{Ci} / \mathrm{g}$ & $\mathrm{Ci} / \mathrm{g}$ & $\mathrm{Ci} / \mathrm{g}$ & $\mathrm{Ci} / \mathrm{g}$ & $\mathrm{Ci} / \mathrm{g}$ \\
\hline Vegetable & $2.4 \mathrm{E}-09$ & $6.7 \mathrm{E}-09$ & $3.6 \mathrm{E}-09$ & $2.1 \mathrm{E}-10$ & $6.1 \mathrm{E}-11$ & $3.4 \mathrm{E}-11$ \\
Wheat grain & $2.4 \mathrm{E}-09$ & $4.7 \mathrm{E}-09$ & $2.6 \mathrm{E}-09$ & $1.5 \mathrm{E}-10$ & $4.4 \mathrm{E}-11$ & $2.5 \mathrm{E}-11$ \\
Meat & $2.4 \mathrm{E}-09$ & $7.7 \mathrm{E}-09$ & $4.1 \mathrm{E}-09$ & $2.3 \mathrm{E}-10$ & $4.7 \mathrm{E}-11$ & $2.8 \mathrm{E}-11$ \\
Milk & $2.4 \mathrm{E}-09$ & $1.4 \mathrm{E}-08$ & $7.7 \mathrm{E}-09$ & $4.4 \mathrm{E}-10$ & $9.3 \mathrm{E}-11$ & $5.4 \mathrm{E}-11$ \\
\hline
\end{tabular}

Table 7. Tritium Concentrations for Average Meteorological Conditions (1 year)

\begin{tabular}{lcccccc}
\hline Pathway & DIL & $0.67 \mathrm{~km}$ & $1 \mathrm{~km}$ & $10 \mathrm{~km}$ & $50 \mathrm{~km}$ & $100 \mathrm{~km}$ \\
& $\mathrm{Ci} / \mathrm{g}$ & $\mathrm{Ci} / \mathrm{g}$ & $\mathrm{Ci} / \mathrm{g}$ & $\mathrm{Ci} / \mathrm{g}$ & $\mathrm{Ci} / \mathrm{g}$ & $\mathrm{Ci} / \mathrm{g}$ \\
\hline Vegetable & $2.4 \mathrm{E}-09$ & $4.8 \mathrm{E}-13$ & $2.6 \mathrm{E}-13$ & $1.4 \mathrm{E}-14$ & $4.0 \mathrm{E}-15$ & $2.2 \mathrm{E}-15$ \\
Wheat grain & $2.4 \mathrm{E}-09$ & $3.1 \mathrm{E}-09$ & $1.7 \mathrm{E}-09$ & $9.7 \mathrm{E}-11$ & $2.9 \mathrm{E}-11$ & $1.6 \mathrm{E}-11$ \\
Meat & $2.4 \mathrm{E}-09$ & $9.2 \mathrm{E}-12$ & $5.0 \mathrm{E}-12$ & $2.8 \mathrm{E}-13$ & $7.9 \mathrm{E}-14$ & $4.4 \mathrm{E}-14$ \\
Milk & $2.4 \mathrm{E}-09$ & $1.5 \mathrm{E}-11$ & $8.0 \mathrm{E}-12$ & $4.5 \mathrm{E}-13$ & $1.3 \mathrm{E}-13$ & $6.9 \mathrm{E}-14$ \\
\hline
\end{tabular}

Table 8. Tritium Concentrations for Adverse Meteorological Conditions (Max HTO)

\begin{tabular}{|c|c|c|c|c|c|c|}
\hline Pathway & $\begin{array}{l}\text { DIL } \\
\mathrm{Ci} / \mathrm{g}\end{array}$ & $\begin{array}{c}0.67 \mathrm{~km} \\
\mathrm{Ci} / \mathrm{g}\end{array}$ & $\begin{array}{l}1 \mathrm{~km} \\
\mathrm{Ci} / \mathrm{g}\end{array}$ & $\begin{array}{c}10 \mathrm{~km} \\
\mathrm{Ci} / \mathrm{g}\end{array}$ & $\begin{array}{c}50 \mathrm{~km} \\
\mathrm{Ci} / \mathrm{g}\end{array}$ & $\begin{array}{c}100 \mathrm{~km} \\
\mathrm{Ci} / \mathrm{g}\end{array}$ \\
\hline Vegetable & $5.9 \mathrm{E}-09$ & $3.0 \mathrm{E}-06$ & $1.7 \mathrm{E}-06$ & $7.1 \mathrm{E}-08$ & $8.7 \mathrm{E}-09$ & $2.5 \mathrm{E}-09$ \\
\hline Wheat grain & $5.9 \mathrm{E}-09$ & 9.4E-07 & 5.4E-07 & 2.2E-08 & $3.9 \mathrm{E}-09$ & $1.1 \mathrm{E}-09$ \\
\hline Meat & $5.9 \mathrm{E}-09$ & 1.1E-07 & $6.5 E-08$ & $2.5 \mathrm{E}-09$ & $2.9 \mathrm{E}-10$ & $1.1 \mathrm{E}-10$ \\
\hline Milk & 5.9E-09 & $1.4 \mathrm{E}-07$ & 8.2E-08 & 3.1E-09 & 3.6E-10 & $1.8 \mathrm{E}-10$ \\
\hline \multicolumn{7}{|c|}{ Table 9. Tritium Concentrations for Adverse Meteorological Conditions (1 week) } \\
\hline Pathway & $\begin{array}{l}\mathrm{DIL} \\
\mathrm{Ci} / \mathrm{g}\end{array}$ & $\begin{array}{c}0.67 \mathrm{~km} \\
\mathrm{Ci} / \mathrm{g}\end{array}$ & $\begin{array}{l}1 \mathrm{~km} \\
\mathrm{Ci} / \mathrm{g}\end{array}$ & $\begin{array}{c}10 \mathrm{~km} \\
\mathrm{Ci} / \mathrm{g}\end{array}$ & $\begin{array}{c}50 \mathrm{~km} \\
\mathrm{Ci} / \mathrm{g}\end{array}$ & $\begin{array}{c}100 \mathrm{~km} \\
\mathrm{Ci} / \mathrm{g}\end{array}$ \\
\hline Vegetable & $2.4 \mathrm{E}-09$ & $4.8 \mathrm{E}-08$ & 2.9E-08 & $1.6 \mathrm{E}-09$ & $3.3 \mathrm{E}-10$ & $1.3 \mathrm{E}-10$ \\
\hline Wheat grain & $2.4 \mathrm{E}-09$ & $2.5 E-08$ & $1.5 E-08$ & 9.5E-10 & 2.3E-10 & $1.1 \mathrm{E}-10$ \\
\hline Meat & 2.4E-09 & 5.1E-08 & 3.1E-08 & $1.6 \mathrm{E}-09$ & $2.8 \mathrm{E}-10$ & $1.5 \mathrm{E}-10$ \\
\hline Milk & 2.4E-09 & 8.7E-08 & $5.2 \mathrm{E}-08$ & 2.8E-09 & 5.2E-10 & 2.6E-10 \\
\hline
\end{tabular}


Table 10. Tritium Concentrations for Adverse Meteorological Conditions (1 year)

\begin{tabular}{lcccccc}
\hline Pathway & DIL & $\begin{array}{c}0.67 \mathrm{~km} \\
\mathrm{Ci} / \mathrm{g}\end{array}$ & $\begin{array}{c}1 \mathrm{~km} \\
\mathrm{Ci} / \mathrm{g}\end{array}$ & $\begin{array}{c}10 \mathrm{~km} \\
\mathrm{Ci} / \mathrm{g}\end{array}$ & $\begin{array}{c}50 \mathrm{~km} \\
\mathrm{Ci} / \mathrm{g}\end{array}$ & $\begin{array}{c}100 \mathrm{~km} \\
\mathrm{Ci} / \mathrm{g}\end{array}$ \\
\hline Vegetable & $2.4 \mathrm{E}-09$ & $2.3 \mathrm{E}-12$ & $1.4 \mathrm{E}-12$ & $7.4 \mathrm{E}-14$ & $1.5 \mathrm{E}-14$ & $5.4 \mathrm{E}-15$ \\
Wheat grain & $2.4 \mathrm{E}-09$ & $1.7 \mathrm{E}-08$ & $1.0 \mathrm{E}-08$ & $5.7 \mathrm{E}-10$ & $1.3 \mathrm{E}-10$ & $5.2 \mathrm{E}-11$ \\
Meat & $2.4 \mathrm{E}-09$ & $5.1 \mathrm{E}-11$ & $3.1 \mathrm{E}-11$ & $1.7 \mathrm{E}-12$ & $3.5 \mathrm{E}-13$ & $1.5 \mathrm{E}-13$ \\
Milk & $2.4 \mathrm{E}-09$ & $7.7 \mathrm{E}-11$ & $4.7 \mathrm{E}-11$ & $2.5 \mathrm{E}-12$ & $5.4 \mathrm{E}-13$ & $2.2 \mathrm{E}-13$ \\
\hline
\end{tabular}

\subsection{CONCLUSIONS}

Tritium concentrations have been calculated for vegetables, wheat grain, meat, and milk under average and adverse meteorological conditions for a postulated full facility fire in SRTC. Peak HTO, one week, and one year concentrations were evaluated and compared with the DILs. Values in Tables 5 to 10 indicate the concentrations, in bold, where the DILs are exceeded.

When the time and distance dependent foodstuff tritium concentrations are compared with the appropriate DILs, the following relationships are noted for each meteorological condition. Under average meteorological conditions, defined as persistent $\mathrm{C}$ atmospheric stability and 2.5 $\mathrm{m} / \mathrm{s}$ windspeed:

- Peak HTO concentrations exceed the DIL for all foodstuffs at $1 \mathrm{~km}$ and vegetables at 10 $\mathrm{km}$

- One week concentrations exceed the DIL for all foodstuffs at $1 \mathrm{~km}$

- At one year, only the concentration of tritium in wheat grain at $0.67 \mathrm{~km}$ (site boundary) exceeds the DIL

Under adverse meteorological conditions, defined as persistent $\mathrm{E}$ atmospheric stability and 1.7 $\mathrm{m} / \mathrm{s}$ windspeed:

- Peak HTO concentrations exceed the DIL for all foodstuffs at $1 \mathrm{~km}$, wheat grain at 10 $\mathrm{km}$, and vegetables at $50 \mathrm{~km}$

- One week concentrations exceed the DIL for all foodtuffs at $1 \mathrm{~km}$ and milk at $10 \mathrm{~km}$

- At one year, the concentration in wheat grain exceeds the DIL at $1 \mathrm{~km}$. 


\subsection{REFERENCES}

FDA (1998). Accidental Contamination of Human Foods and Animal Feeds: Recommendations for States and Local Agencies. Food and Drug Administration, Rockville, MD.

D.J. Hadlock (1997). Emergency Preparedness Hazards Assessment for the SRTC TA, Westinghouse Savannah River Company, Aiken, SC, S-EHA-A-00001, Rev. 1.

W. Raskob (1990). UFOTRI: Program for Assessing the Off-Site Consequences from Accidental Tritium Releases. Kernforschungszentrum Karlsruhe, KfK 4605.

W. Raskob (1993). Description of the New Version 4.0 of the Tritium Model UFOTRI Including User Guide. Kernforschungszentrum Karlsruhe, KfK 5194.

B.K. Tharakan (1999). Derived Intervention levels for Tritium Based on Food and Drug Administration Methodology Using ICRP 56 Dose Coefficients $(U)$. Westinghouse Savannah River Company, Aiken, SC, WSRC-TR-99-00064.

J.M. Thompson (1999a). Ingestion Pathway Consequences of a Major Release from SRTC (U), Westinghouse Savannah River Company, Aiken, SC, WSRC-TR-99-00090, Rev. 0.

J.M. Thompson (1999b). Total Airborne Release for SRTC Accident Scenario 1-RD-3 (U), Westinghouse Savannah River Company, Aiken, SC, S-CLC-A-00104, Rev. 0. 


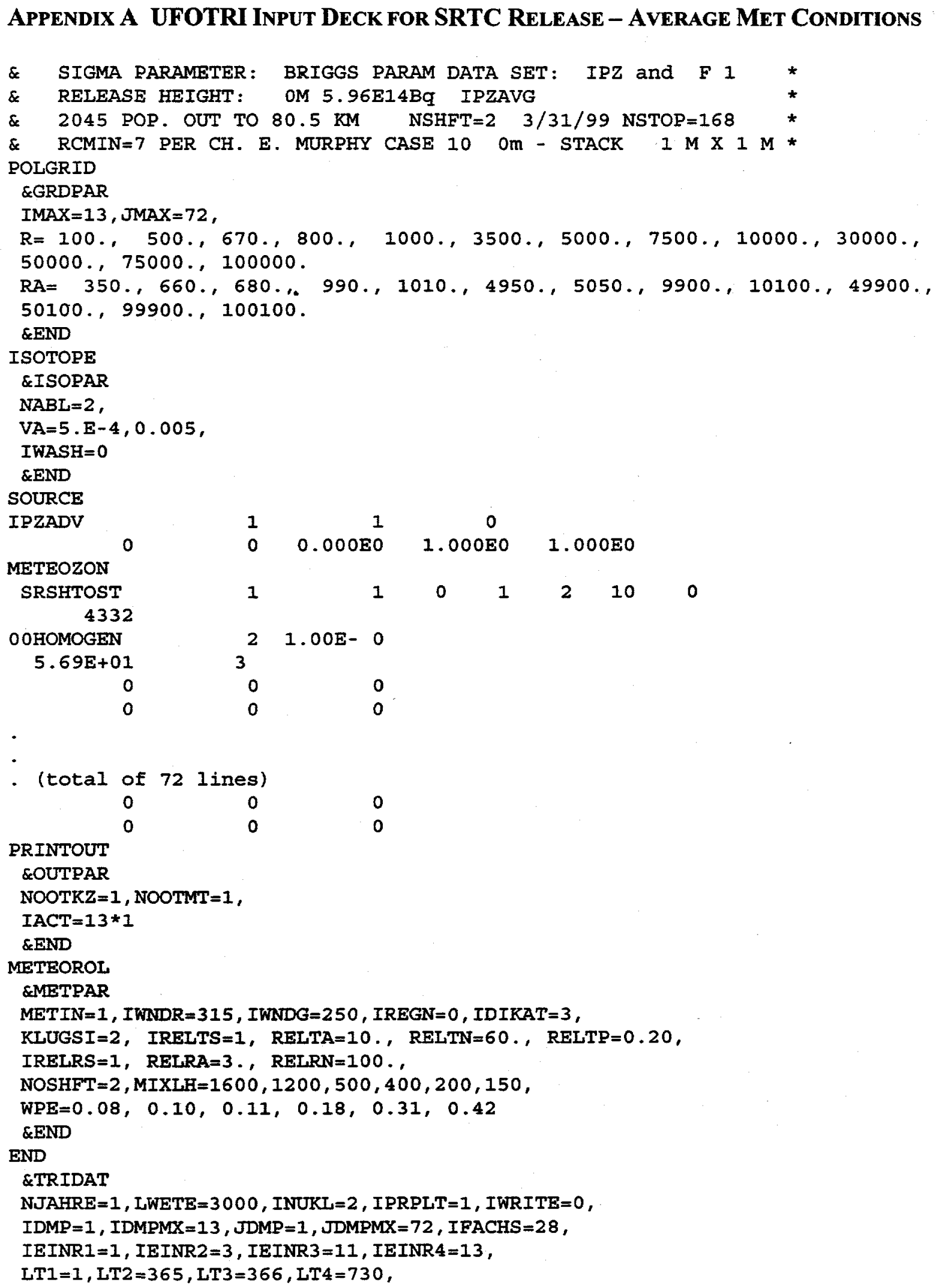


NSTOP $=70, B O D F A K=1 . E+2$, REMRE $=0.3$, IZFREI $=3600, I F L E N G=0, B O D E I N=0.35$, IRFIX $=0$, REFEST $=5.0$, ITEMPE $=3222$, ISTRBE $=200$, ISTRGR $=200$, RELFA $=0.8, A R A U=0.1$, IZUSD $=0$, IZUAUS $=0, \mathrm{KAUSDR}=0,1,0,0,0,0,0,0,0,0,0,0$, $Q(2,1)=1.0, Q(1,1)=0.0, Q 1=5.96 \mathrm{E} 14$, NBOX $=10$, NINI $=10$, NINT $=10$, NOUT $=10$, NTIM $=3, \operatorname{ITIMD}=9, \mathrm{NCOW}=250$, WWCOW $=350$, WOCOW $=150$.

TIMEDO $=0,3,3 \ldots, 14 \ldots, 21,30,90 \ldots, 180,200 \ldots, 365 \ldots, 370 \ldots, 3650 \ldots$ $F A K C 1=0.5, F A K C 2=0.35, F A K C P=0.05, F A K C W=0.1$, RCMIN $=7$, , LEAF $=5$, , BEWG $=269 ., B E W G Z=156 .$, RCMIN2 $=2$, , LEAF2 $=3$, , BEWG2 $=680$, BEWGZ2 $=170 \ldots$ RCMINP $=2$. , LEAFP $=4.7$, BEWGP $=2700 ., B E W G Z P=300$. , RCMINW $=2$. , LEAFW $=7$. , BEWGW $=2000$, , BEWGZW $=500$. , IPOT $=233$, IWHEAT $=261$, IMILK=315, IVEGE $=124$, IMEAT $=206$, DOSF $=1.7 E-11$, DOSHT $=1.7 \mathrm{E}-15, \mathrm{DOSFOB}=4.0 \mathrm{E}-11, \mathrm{BRRATE}=3.33 \mathrm{E}-4, \mathrm{SKRATE}=1.665 \mathrm{E}-04$, IMODEL $=1$, IARTBO $=2$, WIITP $=0.1$, SOILMX $=0.5$, IBODEX $=1$, IMONT $=1$, IRRIGA $=0$, IRRIGG $=0$, IFLAGS $=1$, IFLAGR $=1$, IDEPHT $=1$, IBODFX $=0, I C O V E R=1, I R E L I N=181$, IPHOTO $=1$, IARIV $=0$ \&END 


\section{APPENDIX B RADIONUCLIDE CONCENTRATIONS IN FOODSTUFFs}

\begin{tabular}{|c|c|c|c|c|c|c|c|c|}
\hline & \multicolumn{6}{|c|}{ SRTC Release Scenario 1-RD-3, Average Met Conditions } & & \\
\hline & \multicolumn{4}{|c|}{ Concentration Values for $670 \mathrm{~m}$ from release } & & & & \\
\hline \multicolumn{9}{|c|}{ Only Wheat Seed is examined since this is the edible portion of the Wheat. The "WHEAT" category is } \\
\hline \multicolumn{9}{|c|}{ actually the unedible plant body and is not considered in the dose estimate. } \\
\hline & & & & & & & & \\
\hline & \multicolumn{2}{|c|}{ VEGETABLES } & \multicolumn{2}{|c|}{ WHEAT SEED } & \multicolumn{2}{|l|}{ MEAT } & \multicolumn{2}{|l|}{ MILK } \\
\hline \multirow[t]{2}{*}{ HOUR } & HTO & OBT & HTO & OBT & HTO & OBT & HTO & OBT \\
\hline & $(\mathrm{Bq} / \mathrm{g})$ & $(\mathrm{Bq} / \mathrm{g})$ & $(\mathrm{Bq} / \mathrm{g})$ & $(\mathrm{Bq} / \mathrm{g})$ & $(\mathrm{Bq} / \mathrm{g})$ & $(\mathrm{Bq}$ & $(\mathrm{Bq} / \mathrm{g})$ & $3 \mathrm{q} / \mathrm{g}$ \\
\hline & & & & & & & & \\
\hline 1 & $1.39 \mathrm{E}$ & 7.3 & 5.021 & $1.91 \mathrm{E}+01$ & 3.59 & 7.63E-02 & $35 E+01$ & \\
\hline 2 & $5.64 \mathrm{E}+03$ & $9.84 \mathrm{E}+01$ & $7.18 \mathrm{E}+03$ & $3.29 \mathrm{E}+01$ & $5.15 \mathrm{E}+02$ & $1.88 \mathrm{E}-01$ & $9.41 \mathrm{E}+01$ & $\overline{4.2}$ \\
\hline 3 & $2.92 \mathrm{E}+03$ & $1.08 \mathrm{E}+02$ & $7.59 \mathrm{E}+03$ & $4.25 \mathrm{E}+01$ & $5.93 \mathrm{E}+02$ & $3.18 \mathrm{E}-01$ & $1.42 \mathrm{E}+02$ & $0.3 / \mathrm{E}+0 \mathrm{H}$ \\
\hline 4 & $1.94 \mathrm{E}+03$ & $1.14 \mathrm{E}+02$ & $7.10 \mathrm{E}+03$ & $4.91 \mathrm{E}+01$ & $6.33 \mathrm{E}+02$ & $4.56 \mathrm{E}-01$ & $1.85 \mathrm{E}+02$ & \\
\hline 5 & $1.53 \mathrm{E}+03$ & $1.17 \mathrm{E}+02$ & $6.22 \mathrm{E}+03$ & $5.36 \mathrm{E}+01$ & $6.56 \mathrm{E}+02$ & $6.01 \mathrm{E}-01$ & $2.23 E+02$ & $1.00 \mathrm{E}+02$ \\
\hline 6 & $1.34 \mathrm{E}+03$ & $1.19 \mathrm{E}+02$ & $5.24 \mathrm{E}+03$ & $5.68 \mathrm{E}+01$ & $6.51 \mathrm{E}+02$ & $6.70 \mathrm{E}-01$ & $6.31 \mathrm{E}+02$ & $1.97 \mathrm{E}+02$ \\
\hline 7 & $1.23 \mathrm{E}+03$ & $1.22 \mathrm{E}+02$ & $4.33 \mathrm{E}+03$ & $5.92 \mathrm{E}+01$ & $6.46 \mathrm{E}+02$ & $7.39 \mathrm{E}-01$ & $29 \mathrm{E}+02$ & $1.96 \mathrm{E}$ \\
\hline 8 & $1.15 \mathrm{E}+03$ & $1.24 \mathrm{E}+02$ & $3.52 \mathrm{E}+03$ & $6.09 \mathrm{E}+01$ & $6.41 \mathrm{E}+02$ & $8.08 \mathrm{E}-01$ & $6.26 \mathrm{E}+02$ & $1.95 \mathrm{E}+$ \\
\hline 9 & $1.09 \mathrm{E}+03$ & $1.26 \mathrm{E}+02$ & $2.86 \mathrm{E}+03$ & $6.23 E+01$ & $6.36 \mathrm{E}+02$ & $8.76 \mathrm{E}-01$ & $6.24 \mathrm{E}+02$ & $1.94 \mathrm{E}+02$ \\
\hline 10 & $1.04 \mathrm{E}+03$ & $1.27 \mathrm{E}+02$ & $2.32 \mathrm{E}+03$ & $6.34 \mathrm{E}+01$ & $6.32 \mathrm{E}+02$ & $9.43 \mathrm{E}-01$ & $6.22 \mathrm{E}+02$ & 1.9 \\
\hline 11 & $9.96 \mathrm{E}+02$ & $1.29 \mathrm{E}+02$ & $1.89 \mathrm{E}+03$ & $6.44 \mathrm{E}+01$ & $6.27 \mathrm{E}+02$ & $1.01 \mathrm{E}+00$ & $6.19 \mathrm{E}+02$ & $1.93 \mathrm{E}-1$ \\
\hline 12 & $9.58 \mathrm{E}+02$ & $1.31 E+02$ & $1.56 \mathrm{E}+03$ & $6.53 \mathrm{E}+01$ & $6.22 \mathrm{E}+02$ & $1.08 \mathrm{E}+00$ & $6.17 \mathrm{E}+02$ & $1.92 \mathrm{E}+0$ \\
\hline 13 & $9.23 \mathrm{E}+02$ & $1.32 \mathrm{E}+02$ & $1.30 \mathrm{E}+03$ & $.60 \mathrm{E}+01$ & $6.17 \mathrm{E}+02$ & $1.14 \mathrm{E}+00$ & $6.14 \mathrm{E}+02$ & $1.92 \mathrm{E}+0$ \\
\hline 14 & $8.90 \mathrm{E}+02$ & $1.34 \mathrm{E}+02$ & $1.10 \mathrm{E}+03$ & $6.67 \mathrm{E}+01$ & $6.12 \mathrm{E}+02$ & $1.21 \mathrm{E}+00$ & $6.12 \mathrm{E}+02$ & $1.91 \mathrm{E}+0$ \\
\hline 15 & $8.59 \mathrm{E}+02$ & $1.35 \mathrm{E}+02$ & $9.49 \mathrm{E}+02$ & $6.73 E+01$ & $6.07 \mathrm{E}+02$ & $1.27 \mathrm{E}+00$ & $6.10 \mathrm{E}+02$ & $1.90 \mathrm{E}+02$ \\
\hline 16 & $8.30 \mathrm{E}+02$ & $1.36 \mathrm{E}+02$ & $8.30 \mathrm{E}+02$ & $6.79 \mathrm{E}+01$ & $6.02 \mathrm{E}+02$ & $1.34 \mathrm{E}+00$ & $6.07 \mathrm{E}+02$ & $1.89 \mathrm{E}+0$ \\
\hline 17 & $8.02 E+02$ & $1.38 \mathrm{E}+02$ & $7.37 \mathrm{E}+02$ & $6.85 \mathrm{E}+01$ & $5.98 \mathrm{E}+02$ & $1.40 \mathrm{E}+00$ & $6.05 \mathrm{E}+02$ & $1.89 \mathrm{E}+0$ \\
\hline 18 & $7.76 \mathrm{E}+02$ & $1.39 \mathrm{E}+02$ & $6.64 \mathrm{E}+02$ & $6.91 \mathrm{E}+01$ & $6.01 \mathrm{E}+02$ & $1.55 \mathrm{E}+00$ & $5.82 \mathrm{E}+02$ & $2.90 \mathrm{E}+0$ \\
\hline 19 & $7.50 \mathrm{E}+02$ & $1.40 \mathrm{E}+02$ & $6.06 \mathrm{E}+02$ & $6.96 \mathrm{E}+01$ & $6.04 \mathrm{E}+02$ & $1.71 \mathrm{E}+00$ & $5.84 \mathrm{E}+02$ & $2.91 \mathrm{E}+02$ \\
\hline 20 & $7.26 \mathrm{E}+02$ & $1.41 \mathrm{E}+02$ & $5.58 \mathrm{E}+02$ & $7.01 \mathrm{E}+01$ & $6.06 \mathrm{E}+02$ & $1.86 \mathrm{E}+00$ & $5.85 \mathrm{E}+02$ & $2.92 \mathrm{E}+02$ \\
\hline 21 & $7.02 \mathrm{E}+02$ & $1.42 \mathrm{E}+02$ & $5.19 \mathrm{E}+02$ & $7.05 \mathrm{E}+01$ & $6.09 \mathrm{E}+02$ & $2.02 \mathrm{E}+00$ & $5.86 \mathrm{E}+02$ & $2.92 \mathrm{E}+02$ \\
\hline 22 & $6.78 \mathrm{E}+02$ & $1.44 \mathrm{E}+02$ & $4.86 \mathrm{E}+02$ & $7.10 \mathrm{E}+01$ & $6.11 \mathrm{E}+02$ & $2.17 \mathrm{E}+00$ & $5.8 \overline{8 E}+02$ & $2.93 \mathrm{E}+02$ \\
\hline 23 & $6.56 \mathrm{E}+02$ & $1.45 \mathrm{E}+02$ & $4.58 \mathrm{E}+02$ & $7.14 \mathrm{E}+01$ & $6.13 E+02$ & $2.33 \mathrm{E}+00$ & $5.89 \mathrm{E}+02$ & $2.94 \mathrm{E}+0$ \\
\hline 24 & $6.35 \mathrm{E}+02$ & $1.46 \mathrm{E}+02$ & $4.33 E+02$ & $7.18 \mathrm{E}+01$ & $6.14 \mathrm{E}+02$ & $2.48 \mathrm{E}+00$ & $5.89 \mathrm{E}+02$ & $2.94 \mathrm{E}+02$ \\
\hline 25 & $6.15 \bar{E}+02$ & $1.47 \mathrm{E}+02$ & $4.11 E+02$ & $7.22 \mathrm{E}+01$ & $6.15 E+02$ & $2.64 \mathrm{E}+00$ & $5.90 \mathrm{E}+02$ & $2.95 \mathrm{E}+02$ \\
\hline 26 & $5.96 \mathrm{E}+02$ & $1.47 \mathrm{E}+02$ & $3.92 \mathrm{E}+02$ & $7.26 \mathrm{E}+01$ & $6.16 \mathrm{E}+02$ & $2.80 \mathrm{E}+00$ & $5.91 \mathrm{E}+02$ & $2.95 \mathrm{E}+$ \\
\hline 27 & $5.77 \mathrm{E}+02$ & $1.48 \mathrm{E}+02$ & $3.73 E+02$ & $7.30 \mathrm{E}+01$ & $6.17 \mathrm{E}+02$ & $2.96 \mathrm{E}+00$ & $5.92 \mathrm{E}+02$ & $2.96 \mathrm{E}+02$ \\
\hline 28 & $5.58 \mathrm{E}+02$ & $1.49 \mathrm{E}+02$ & $3.57 \mathrm{E}+02$ & $7.33 \mathrm{E}+01$ & $6.18 \mathrm{E}+02$ & $3.12 \mathrm{E}+00$ & $5.92 \mathrm{E}+02$ & $2.96 \mathrm{E}+02$ \\
\hline 29 & $5.41 \mathrm{E}+02$ & $1.50 \mathrm{E}+02$ & $3.41 \mathrm{E}+02$ & $7.37 \mathrm{E}+01$ & $6.18 \mathrm{E}+02$ & $3.27 \mathrm{E}+00$ & $5.93 \mathrm{E}+02$ & 2.97 \\
\hline 30 & $5.25 \mathrm{E}+02$ & $1.51 \mathrm{E}+02$ & $3.26 \mathrm{E}+02$ & $7.40 \mathrm{E}+01$ & $6.13 E+02$ & $3.34 \mathrm{E}+00$ & $5.94 \mathrm{E}+02$ & $1.86 \mathrm{E}+02$ \\
\hline 31 & $4.96 \mathrm{E}+02$ & $1.51 E+02$ & $3.13 E+02$ & $7.43 E+01$ & $6.08 \mathrm{E}+02$ & $3.40 \mathrm{E}+00$ & $5.92 \mathrm{E}+02$ & $1.85 \mathrm{E}+02$ \\
\hline 32 & $4.79 \mathrm{E}+02$ & $1.52 \mathrm{E}+02$ & $2.98 \mathrm{E}+02$ & $7.46 \mathrm{E}+01$ & $6.03 \mathrm{E}+02$ & $3.46 \mathrm{E}+00$ & $5.89 \mathrm{E}+02$ & $1.84 \mathrm{E}+02$ \\
\hline 33 & $4.65 \mathrm{E}+02$ & $1.53 \mathrm{E}+02$ & $2.84 \mathrm{E}+02$ & $7.49 \mathrm{E}+01$ & $5.98 \mathrm{E}+02$ & $3.53 \mathrm{E}+00$ & $5.87 \mathrm{E}+02$ & $1.83 \mathrm{E}+02$ \\
\hline 34 & $4.53 E+02$ & $1.53 E+02$ & $2.70 \bar{E}+02$ & $7.51 \mathrm{E}+01$ & $5.93 E+02$ & $3.59 \mathrm{E}+00$ & $5.85 \mathrm{E}+02$ & $1.83 E+02$ \\
\hline 35 & $4.42 \mathrm{E}+02$ & $1.54 \mathrm{E}+02$ & $2.58 \mathrm{E}+02$ & $7.54 \mathrm{E}+01$ & $5.88 \mathrm{E}+02$ & $3.65 \mathrm{E}+00$ & $5.82 \mathrm{E}+02$ & $1.82 \mathrm{E}+02$ \\
\hline 36 & $4.31 \mathrm{E}+02$ & $1.55 \mathrm{E}+02$ & $2.47 \mathrm{E}+02$ & $7.56 \mathrm{E}+01$ & $5.84 \mathrm{E}+02$ & $3.71 \mathrm{E}+00$ & $5.80 \mathrm{E}+02$ & $1.81 \mathrm{E}+02$ \\
\hline 37 & $4.21 \mathrm{E}+02$ & $1.55 \mathrm{E}+02$ & $2.36 \mathrm{E}+02$ & $7.59 \mathrm{E}+01$ & $5.79 \mathrm{E}+02$ & $3.77 \mathrm{E}+00$ & $5.77 \mathrm{E}+02$ & $1.81 \mathrm{E}+02$ \\
\hline
\end{tabular}




\begin{tabular}{|c|c|c|c|c|c|c|c|c|}
\hline 38 & $.13 \mathrm{E}+02$ & $1.56 \mathrm{E}+02$ & $2.26 \mathrm{E}+02$ & $7.61 \mathrm{E}+01$ & $5.74 \mathrm{E}+02$ & $3.83 \mathrm{E}+00$ & $5.75 \mathrm{E}+02$ & \\
\hline 39 & $05 \mathrm{E}+02$ & $1.56 \mathrm{E}+02$ & $2.18 \mathrm{E}+02$ & $7.63 \mathrm{E}+01$ & $5.69 \mathrm{E}+02$ & $3.89 \mathrm{E}+00$ & $5.73 \mathrm{E}+02$ & $1.79 \mathrm{E}+02$ \\
\hline 40 & $3.97 \mathrm{E}+02$ & $1.57 \mathrm{E}+02$ & $2.10 \mathrm{E}+02$ & $7.65 \mathrm{E}+01$ & $5.65 \mathrm{E}+02$ & $3.94 \mathrm{E}+00$ & $.70 \mathrm{E}+02$ & $1.78 \mathrm{E}+02$ \\
\hline 41 & $90 \mathrm{E}+02$ & $1.57 \mathrm{E}+02$ & $.03 \mathrm{E}+02$ & $.67 \mathrm{E}+01$ & $.60 \mathrm{E}+02$ & $4.00 E+00$ & $.68 \mathrm{E}+02$ & $.78 \mathrm{E}+0$ \\
\hline 42 & $3.83 \mathrm{E}+02$ & $1.58 \mathrm{E}+02$ & $1.96 \mathrm{E}+02$ & $7.69 \mathrm{E}+01$ & $5.59 \mathrm{E}+02$ & $4.16 \mathrm{E}+00$ & $5.42 \mathrm{E}+02$ & $2.90 \mathrm{E}+0$ \\
\hline 43 & $3.76 \mathrm{E}+02$ & $1.58 \mathrm{E}+02$ & $1.90 \mathrm{E}+02$ & $7.71 \mathrm{E}+01$ & $5.58 \mathrm{E}+02$ & $4.31 \mathrm{E}+00$ & $5.41 E+02$ & $.90 \mathrm{E}+02$ \\
\hline 44 & $3.70 \mathrm{E}+02$ & $1.59 \mathrm{E}+02$ & $1.84 \mathrm{E}+02$ & $7.73 \mathrm{E}+01$ & $5.57 \mathrm{E}+02$ & $4.47 \mathrm{E}+00$ & $5.41 E+02$ & $2.89 \mathrm{E}+\mathrm{C}$ \\
\hline 45 & $3.51 \mathrm{E}+02$ & $1.59 \mathrm{E}+02$ & $1.79 \mathrm{E}+02$ & $7.75 \mathrm{E}+01$ & $5.56 \mathrm{E}+02$ & $4.63 \mathrm{E}+00$ & $5.41 \mathrm{E}+02$ & $2.89 \mathrm{E}+0$ \\
\hline 46 & $3.43 E+02$ & $1.60 \mathrm{E}+02$ & $1.73 E+02$ & $7.77 \mathrm{E}+01$ & $5.55 \mathrm{E}+02$ & $4.78 \mathrm{E}+00$ & $5.40 \mathrm{E}+02$ & $2.89 \mathrm{E}+0$ \\
\hline 47 & $3.38 \mathrm{E}+02$ & $1.60 \mathrm{E}+02$ & $1.66 \mathrm{E}+02$ & $7.79 \mathrm{E}+01$ & $5.54 \mathrm{E}+02$ & $4.94 \mathrm{E}+00$ & $5.39 \mathrm{E}+02$ & $2.89 \mathrm{E}+0$ \\
\hline 48 & $3.31 \mathrm{E}+02$ & $1.60 \mathrm{E}+02$ & $1.60 \mathrm{E}+02$ & $7.80 \mathrm{E}+01$ & $5.52 \mathrm{E}+02$ & $5.09 \mathrm{E}+00$ & $5.39 \mathrm{E}+02$ & $2.89 \mathrm{E}+0$ \\
\hline 49 & $3.27 \mathrm{E}+02$ & $1.61 \mathrm{E}+02$ & $1.54 \mathrm{E}+02$ & $7.82 \mathrm{E}+01$ & $5.51 \mathrm{E}+02$ & $5.25 \mathrm{E}+00$ & $.38 \mathrm{E}+02$ & $2.89 \mathrm{E}+0$ \\
\hline 50 & $3.23 E+02$ & $1.61 \mathrm{E}+02$ & $1.49 \mathrm{E}+02$ & $7.83 \mathrm{E}+01$ & $5.50 \mathrm{E}+02$ & $5.40 \mathrm{E}+00$ & $.38 \mathrm{E}+02$ & $2.89 \mathrm{E}+0$ \\
\hline 51 & $3.20 \mathrm{E}+02$ & $1.61 \mathrm{E}+02$ & $1.45 \mathrm{E}+02$ & $7.85 \mathrm{E}+01$ & $5.48 \mathrm{E}+02$ & $5.56 \mathrm{E}+00$ & $5.37 \mathrm{E}+02$ & $2.89 \mathrm{E}+02$ \\
\hline 52 & $3.17 \mathrm{E}+02$ & $1.62 \mathrm{E}+02$ & $1.41 \mathrm{E}+02$ & $7.86 \mathrm{E}+01$ & $.47 \mathrm{E}+02$ & $5.71 \mathrm{E}+00$ & $36 \mathrm{E}+02$ & $2.89 \mathrm{E}+0$ \\
\hline 53 & $3.15 \mathrm{E}+02$ & $1.62 \mathrm{E}+02$ & $1.37 \mathrm{E}+02$ & $7.88 \mathrm{E}+01$ & $5.45 \mathrm{E}+02$ & $.87 \mathrm{E}+00$ & $5.36 \mathrm{E}+02$ & $2.88 \mathrm{E}+0$ \\
\hline 54 & $3.12 \mathrm{E}+02$ & $1.63 E+02$ & $1.34 \mathrm{E}+02$ & $7.89 \mathrm{E}+01$ & $5.41 \mathrm{E}+02$ & $5.92 \mathrm{E}+00$ & $5.24 \mathrm{E}+02$ & $1.64 \mathrm{E}+0$ \\
\hline 55 & $3.09 \mathrm{E}+02$ & $1.63 \mathrm{E}+02$ & $1.32 \mathrm{E}+02$ & $7.90 \mathrm{E}+01$ & $5.36 \mathrm{E}+02$ & $5.97 \mathrm{E}+00$ & $22 \mathrm{E}+02$ & $1.64 \mathrm{E}+0$ \\
\hline 56 & $07 \mathrm{E}+02$ & $1.63 \mathrm{E}+02$ & $1.29 \mathrm{E}+02$ & $7.92 \mathrm{E}+01$ & $5.32 \mathrm{E}+02$ & $6.03 E+00$ & $5.20 \mathrm{E}+02$ & $1.63 \mathrm{E}+0$ \\
\hline 57 & $3.05 \mathrm{E}+02$ & $1.64 \mathrm{E}+02$ & $1.27 \mathrm{E}+02$ & $7.93 \mathrm{E}+01$ & $5.27 \mathrm{E}+02$ & $6.08 \mathrm{E}+00$ & $5.18 \mathrm{E}+02$ & $1.62 \mathrm{E}+02$ \\
\hline 58 & $3.02 \mathrm{E}+02$ & $1.64 \mathrm{E}+02$ & $1.26 \mathrm{E}+02$ & $7.95 \mathrm{E}+01$ & $5.23 \mathrm{E}+02$ & $6.13 E+00$ & $5.15 \mathrm{E}+02$ & $1.62 \mathrm{E}+0$ \\
\hline 59 & $3.00 \mathrm{E}+02$ & $1.64 \mathrm{E}+02$ & $1.24 \mathrm{E}+02$ & $7.96 \mathrm{E}+01$ & $5.19 \mathrm{E}+02$ & $6.18 \mathrm{E}+00$ & $.13 \mathrm{E}+02$ & $1.61 \mathrm{E}+0$ \\
\hline 60 & $2.98 \mathrm{E}+02$ & $1.64 \mathrm{E}+02$ & $1.22 \mathrm{E}+02$ & $7.97 \mathrm{E}+01$ & $5.14 \mathrm{E}+02$ & $6.23 \mathrm{E}+00$ & $5.11 \mathrm{E}+02$ & $1.60 \mathrm{E}+0$ \\
\hline 61 & $2.96 \mathrm{E}+02$ & $1.65 \mathrm{E}+02$ & $1.21 \mathrm{E}+02$ & $7.98 \mathrm{E}+01$ & $5.10 \mathrm{E}+02$ & $6.28 \mathrm{E}+00$ & $5.09 \mathrm{E}+02$ & $1.60 \mathrm{E}+0$ \\
\hline 62 & $2.94 \mathrm{E}+02$ & $1.65 \mathrm{E}+02$ & $1.20 \mathrm{E}+02$ & $8.00 \mathrm{E}+01$ & $5.06 \mathrm{E}+02$ & $6.33 \mathrm{E}+00$ & $5.07 \mathrm{E}+02$ & $1.59 \mathrm{E}+0$ \\
\hline 63 & $2.92 \mathrm{E}+02$ & $1.65 \mathrm{E}+02$ & $1.18 \mathrm{E}+02$ & $8.01 \mathrm{E}+01$ & $5.02 \mathrm{E}+02$ & $6.38 \mathrm{E}+00$ & $5.05 \mathrm{E}+02$ & $1.58 \mathrm{E}+02$ \\
\hline 64 & $2.90 \mathrm{E}+02$ & $1.66 \mathrm{E}+02$ & $1.17 \mathrm{E}+02$ & $8.02 \mathrm{E}+01$ & $4.97 \mathrm{E}+02$ & $6.43 \mathrm{E}+00$ & $.03 E+02$ & $1.58 \mathrm{E}+0$. \\
\hline 65 & $2.88 \mathrm{E}+02$ & $1.66 \mathrm{E}+02$ & $1.16 \mathrm{E}+02$ & $8.04 \mathrm{E}+01$ & $4.93 \mathrm{E}+02$ & $6.48 \mathrm{E}+00$ & $5.01 \mathrm{E}+02$ & $1.57 \mathrm{E}+02$ \\
\hline 66 & $2.86 \mathrm{E}+02$ & $1.66 \mathrm{E}+02$ & $1.15 \mathrm{E}+02$ & $8.05 \mathrm{E}+01$ & $4.92 \mathrm{E}+02$ & $6.63 \mathrm{E}+00$ & $4.77 \mathrm{E}+02$ & $2.73 \mathrm{E}+0$ \\
\hline 67 & $2.84 \mathrm{E}+02$ & $1.67 \mathrm{E}+02$ & $1.14 \mathrm{E}+02$ & $8.06 \mathrm{E}+01$ & $4.91 \mathrm{E}+02$ & $6.78 \mathrm{E}+00$ & $4.76 \mathrm{E}+02$ & $2.73 \mathrm{E}+0$ \\
\hline 68 & $2.82 \mathrm{E}+02$ & $1.67 \mathrm{E}+02$ & $1.13 \mathrm{E}+02$ & $8.07 \mathrm{E}+01$ & $4.89 \mathrm{E}+02$ & $6.93 E+00$ & $4.75 \mathrm{E}+02$ & $2.73 E$ \\
\hline 69 & $81 \mathrm{E}+02$ & $1.67 \mathrm{E}+02$ & $1.12 \mathrm{E}+02$ & $8.09 \mathrm{E}+01$ & $4.88 \mathrm{E}+02$ & $7.08 \mathrm{E}+00$ & $4.75 \mathrm{E}+02$ & $2.73 \mathrm{E}+0$ \\
\hline 168 & $8.16 \mathrm{E}+01$ & $1.68 \mathrm{E}+02$ & $8.48 \mathrm{E}+01$ & $9.03 \mathrm{E}+01$ & $2.64 \mathrm{E}+02$ & $1.97 \mathrm{E}+01$ & $3.10 \mathrm{E}+02$ & $2.20 \mathrm{E}+0$ \\
\hline 336 & $4.66 \mathrm{E}+01$ & $1.42 \mathrm{E}+02$ & $4.74 \mathrm{E}+01$ & $9.70 \mathrm{E}+01$ & $1.14 \mathrm{E}+02$ & $3.38 \mathrm{E}+01$ & $1.72 \mathrm{E}+02$ & $1.61 \mathrm{E}+0$ \\
\hline 504 & $3.49 \mathrm{E}+01$ & $1.19 \mathrm{E}+02$ & $3.30 \mathrm{E}+01$ & $1.01 \mathrm{E}+02$ & $5.82 \mathrm{E}+01$ & $4.15 \mathrm{E}+01$ & $7.96 \mathrm{E}+01$ & $1.11 \mathrm{E}+0$ \\
\hline 720 & $2.56 \mathrm{E}+01$ & $9.42 \mathrm{E}+01$ & $2.23 \mathrm{E}+01$ & $1.05 \mathrm{E}+02$ & $3.03 \mathrm{E}+01$ & $4.56 \mathrm{E}+01$ & $4.10 \mathrm{E}+01$ & $7.79 \mathrm{E}+0$ \\
\hline 160 & $84 \mathrm{E}+00$ & $1.92 \mathrm{E}+01$ & $2.53 \mathrm{E}+00$ & $1.12 \mathrm{E}+02$ & $2.78 \mathrm{E}+00$ & $2.68 \mathrm{E}+01$ & $9.59 \mathrm{E}+00$ & $2.71 \mathrm{E}+01$ \\
\hline 4320 & $4.65 \mathrm{E}-01$ & $1.77 \mathrm{E}+00$ & $1.27 \mathrm{E}+01$ & $1.06 \mathrm{E}+02$ & $4.75 \mathrm{E}-01$ & $6.43 \mathrm{E}+00$ & $1.20 \mathrm{E}+00$ & $4.30 \mathrm{E}+0$ \\
\hline 4800 & $2.77 \mathrm{E}-01$ & $1.05 \mathrm{E}+00$ & $1.26 \mathrm{E}+01$ & $1.06 \mathrm{E}+02$ & $3.46 \mathrm{E}-01$ & $4.63 \mathrm{E}+00$ & $4.07 \mathrm{E}-01$ & $1.42 \mathrm{E}+0$ \\
\hline 8760 & $3.80 \mathrm{E}-03$ & $1.39 \mathrm{E}-02$ & $1.23 \mathrm{E}+01$ & $1.03 E+02$ & $2.88 \mathrm{E}-02$ & $3.12 \mathrm{E}-01$ & $1.28 \mathrm{E}-01$ & $4.20 \mathrm{E}$ \\
\hline & \multicolumn{2}{|c|}{ VEGETABLES } & \multicolumn{2}{|c|}{ WHEAT SEED } & \multicolumn{2}{|l|}{ MEAT } & \multicolumn{2}{|l|}{ MILK } \\
\hline \multirow[t]{2}{*}{ HOUR } & HTO & OBT & $\bar{O}$ & $\overline{\mathrm{OBT}}$ & HTO & $\overline{B T}$ & HTO & OBT \\
\hline & $(\mathrm{Bq} / \mathrm{g})$ & $(\mathrm{Bq} / \mathrm{g})$ & $(\mathrm{g})$ & $(\mathrm{Bq} / \mathrm{g})$ & $(\mathrm{Bq} / \mathrm{g})$ & $(\mathrm{Bq} / \mathrm{g})$ & $(\mathrm{Bq} / \mathrm{g})$ & $(\mathrm{Bq} / \mathrm{g})$ \\
\hline \multicolumn{9}{|l|}{ eak HTO } \\
\hline $1 / 3 / 5 / 6$ & $1.39 \mathrm{E}+04$ & +01 & $7.59 \mathrm{E}+03$ & 4.2 & $6.56 \mathrm{E}+02$ & 6 & $6.31 \mathrm{E}+02$ & \\
\hline UM & $1.40 \mathrm{E}+04$ & & $7.64 \mathrm{E}+03$ & & $6.57 \mathrm{E}+02$ & & $8.28 E+02$ & \\
\hline$\sqrt{g}$ & $3.77 \mathrm{E}-07$ & & 2.06E-07 & & $1.77 \mathrm{E}-08$ & & $2.24 \mathrm{E}-08$ & \\
\hline
\end{tabular}




\begin{tabular}{|c|c|c|c|c|c|c|c|c|}
\hline DIL HTO & $5.9 \mathrm{E}-09$ & & & & & & & \\
\hline \multicolumn{9}{|l|}{$(\mathrm{Ci} / \mathrm{g})$} \\
\hline 168 & $8.16 \mathrm{E}+01$ & $1.68 \mathrm{E}+02$ & $8.48 \mathrm{E}+01$ & $9.03 E+01$ & $2.64 \mathrm{E}+02$ & $1.97 \mathrm{E}+01$ & $3.10 E+02$ & $2.20 \mathrm{E}+02$ \\
\hline SUM & $2.49 \mathrm{E}+02$ & & $1.75 \mathrm{E}+02$ & & $2.84 \mathrm{E}+02$ & & $5.31 \mathrm{E}+02$ & \\
\hline $\mathrm{Ci} / \mathrm{g}$ & $6.73 E-09$ & & $4.73 \mathrm{E}-09$ & & $7.67 \mathrm{E}-09$ & & $1.43 \mathrm{E}-08$ & \\
\hline & & & & & & & & \\
\hline DIL OBT & $2.4 \mathrm{E}-09$ & & & & & & & \\
\hline \multicolumn{9}{|l|}{$\mathrm{Ci} / \mathrm{g}$} \\
\hline & & & & & & & & \\
\hline 8760 & $3.80 \mathrm{E}-03$ & $1.39 \mathrm{E}-02$ & $1.23 \mathrm{E}+01$ & $1.03 E+02$ & $2.88 \mathrm{E}-02$ & $3.12 \mathrm{E}-01$ & $1.28 \mathrm{E}-01$ & $4.20 \mathrm{E}-01$ \\
\hline SUM & $1.77 \mathrm{E}-02$ & & $1.16 \mathrm{E}+02$ & & $3.41 \mathrm{E}-01$ & & $5.48 \mathrm{E}-01$ & \\
\hline $\mathrm{Ci} / \mathrm{g}$ & $4.79 \mathrm{E}-13$ & & 3.12E-09 & & $9.20 \mathrm{E}-12$ & & $1.48 \mathrm{E}-11$ & \\
\hline DIL OBT & $? 1500$ & & & & & & & \\
\hline & $2.4 \mathrm{E}-0 \mathrm{y}$ & & & & & & & \\
\hline $\mathrm{Ci} / \mathrm{g}$ & & & & & & . & & \\
\hline & & & & & & & & \\
\hline
\end{tabular}




\begin{tabular}{|c|c|c|c|c|c|c|c|c|}
\hline & \multicolumn{6}{|c|}{ SRTC Release Scenario 1-RD-3, Average Met Conditions } & & \\
\hline & \multicolumn{4}{|c|}{ Concentration Values for $1 \mathrm{~km}$ from release } & & & & \\
\hline \multicolumn{9}{|c|}{ Only Wheat Seed is examined since this is the edible portion of the Wheat. The "WHEAT" category is } \\
\hline \multicolumn{9}{|c|}{ actually the unedible plant body and is not considered in the dose estimate. } \\
\hline & & & & & & & & \\
\hline & \multicolumn{2}{|c|}{ VEGETABLES } & \multicolumn{2}{|c|}{ WHEAT SEED } & \multicolumn{2}{|l|}{ MEAT } & \multicolumn{2}{|l|}{ MILK } \\
\hline \multirow[t]{2}{*}{ HOUR } & HTO & OBT & HTO & OBT & HTO & OBT & HTO & OBT \\
\hline & $(\mathrm{Bq} / \mathrm{g})$ & $(\mathrm{Bq} / \mathrm{g})$ & $(\mathrm{Bq} / \mathrm{g})$ & $(\mathrm{Bq} / \mathrm{g})$ & $(\mathrm{Bq} / \mathrm{g})$ & $(\mathrm{Bq} / \mathrm{g})$ & $(\mathrm{Bq} / \mathrm{g})$ & $(\mathrm{Bq} / \mathrm{g})$ \\
\hline & & & & & & & & \\
\hline 1 & $7.43 \mathrm{E}+03$ & $3.76 \mathrm{E}+01$ & $2.56 \mathrm{E}+03$ & $9.75 \mathrm{E}+00$ & $1.83 \mathrm{E}+02$ & $3.89 \mathrm{E}-02$ & $2.22 \mathrm{E}+01$ & $9.83 \mathrm{E}+00$ \\
\hline 2 & $3.16 \mathrm{E}+03$ & $5.08 \mathrm{E}+01$ & $3.70 \mathrm{E}+03$ & $1.69 \mathrm{E}+01$ & $2.66 \mathrm{E}+02$ & $9.66 \mathrm{E}-02$ & $4.84 \mathrm{E}+01$ & $2.16 \mathrm{E}+01$ \\
\hline 3 & $1.69 \mathrm{E}+03$ & $5.64 \mathrm{E}+01$ & $3.95 \mathrm{E}+03$ & $2.20 \mathrm{E}+01$ & $3.08 \mathrm{E}+02$ & $1.64 \mathrm{E}-01$ & $7.34 \mathrm{E}+01$ & $3.29 \mathrm{E}+01$ \\
\hline 4 & $1.14 \mathrm{E}+03$ & $5.94 \mathrm{E}+01$ & $3.72 \mathrm{E}+03$ & $2.55 \mathrm{E}+01$ & $3.31 E+02$ & $2.36 \mathrm{E}-01$ & $9.59 E+01$ & $4.30 \mathrm{E}+01$ \\
\hline 5 & $9.08 \mathrm{E}+02$ & $6.13 E+01$ & $3.28 \mathrm{E}+03$ & $2.80 \mathrm{E}+01$ & $3.44 \mathrm{E}+02$ & $3.12 \mathrm{E}-01$ & $1.16 \mathrm{E}+02$ & $5.20 \mathrm{E}+01$ \\
\hline 6 & $7.92 \mathrm{E}+02$ & $6.29 \mathrm{E}+01$ & $2.79 \mathrm{E}+03$ & $2.98 \mathrm{E}+01$ & $3.42 \mathrm{E}+02$ & $3.49 \mathrm{E}-01$ & $3.31 \mathrm{E}+02$ & $1.03 \mathrm{E}+02$ \\
\hline 7 & $7.23 \mathrm{E}+02$ & $6.42 \mathrm{E}+01$ & $2.32 \mathrm{E}+03$ & $3.11 \mathrm{E}+01$ & $3.39 \mathrm{E}+02$ & $3.85 \mathrm{E}-01$ & $3.30 \mathrm{E}+02$ & $1.03 \mathrm{E}+02$ \\
\hline 8 & $6.76 \mathrm{E}+02$ & $6.54 \mathrm{E}+01$ & $1.90 \mathrm{E}+03$ & $3.21 \mathrm{E}+01$ & $3.37 \mathrm{E}+02$ & $4.21 \mathrm{E}-01$ & $3.29 \mathrm{E}+02$ & $1.02 \mathrm{E}+02$ \\
\hline 9 & $6.41 \mathrm{E}+02$ & $6.65 \mathrm{E}+01$ & $1.56 \mathrm{E}+03$ & $3.29 \mathrm{E}+01$ & $3.34 \mathrm{E}+02$ & $4.56 \mathrm{E}-01$ & $3.28 \mathrm{E}+02$ & $1.02 \mathrm{E}+02$ \\
\hline 10 & $6.12 \mathrm{E}+02$ & $6.76 \mathrm{E}+01$ & $1.28 \mathrm{E}+03$ & $3.36 \mathrm{E}+01$ & $3.32 \mathrm{E}+02$ & $4.92 \mathrm{E}-01$ & $3.26 \mathrm{E}+02$ & $1.02 \mathrm{E}+02$ \\
\hline 11 & $5.87 \mathrm{E}+02$ & $6.86 E+01$ & $1.05 \mathrm{E}+03$ & $3.41 \mathrm{E}+01$ & $3.29 \mathrm{E}+02$ & $5.27 \mathrm{E}-01$ & $3.25 E+02$ & $1.01 \mathrm{E}+02$ \\
\hline 12 & $5.64 \mathrm{E}+02$ & $6.96 \mathrm{E}+01$ & $8.75 \mathrm{E}+02$ & $3.46 \mathrm{E}+01$ & $3.27 \mathrm{E}+02$ & $5.62 \mathrm{E}-01$ & $3.24 \mathrm{E}+02$ & $1.01 \mathrm{E}+02$ \\
\hline 13 & $5.43 E+02$ & $7.05 E+01$ & $7.38 \mathrm{E}+02$ & $3.51 \mathrm{E}+01$ & $3.24 \mathrm{E}+02$ & $5.96 \mathrm{E}-01$ & $3.23 \mathrm{E}+02$ & $1.01 \mathrm{E}+02$ \\
\hline 14 & $5.24 \mathrm{E}+02$ & $7.14 \mathrm{E}+01$ & $6.32 \mathrm{E}+02$ & $3.55 \mathrm{E}+01$ & $3.22 \mathrm{E}+02$ & $6.30 \mathrm{E}-01$ & $3.21 \mathrm{E}+02$ & $1.00 \mathrm{E}+02$ \\
\hline 15 & $5.06 \mathrm{E}+02$ & $7.22 \mathrm{E}+01$ & $5.49 \mathrm{E}+02$ & $3.59 \mathrm{E}+01$ & $3.19 \mathrm{E}+02$ & 6.64E-01 & $3.20 \mathrm{E}+02$ & $9.98 \mathrm{E}+01$ \\
\hline 16 & $4.89 \mathrm{E}+02$ & $7.30 \mathrm{E}+01$ & $4.85 \mathrm{E}+02$ & $3.62 \mathrm{E}+01$ & $3.17 \mathrm{E}+02$ & $6.98 \mathrm{E}-01$ & $3.19 \mathrm{E}+02$ & $9.94 \mathrm{E}+01$ \\
\hline 17 & $4.72 \mathrm{E}+02$ & $7.38 \mathrm{E}+01$ & $4.34 \mathrm{E}+02$ & $3.66 \mathrm{E}+01$ & $3.14 \mathrm{E}+02$ & $7.31 \mathrm{E}-01$ & $3.18 \mathrm{E}+02$ & $9.90 \mathrm{E}+01$ \\
\hline 18 & $4.57 \mathrm{E}+02$ & $7.45 \mathrm{E}+01$ & $3.94 E+02$ & $3.69 \mathrm{E}+01$ & $3.16 \mathrm{E}+02$ & $8.12 \mathrm{E}-01$ & $3.07 \mathrm{E}+02$ & $1.54 \mathrm{E}+02$ \\
\hline 19 & $4.42 \mathrm{E}+02$ & $7.53 \mathrm{E}+01$ & $3.61 \mathrm{E}+02$ & $3.72 \mathrm{E}+01$ & $3.18 \mathrm{E}+02$ & $8.94 \mathrm{E}-01$ & $3.08 \mathrm{E}+02$ & $1.54 \mathrm{E}+02$ \\
\hline 20 & $4.23 \mathrm{E}+02$ & $7.59 \mathrm{E}+01$ & $3.35 \mathrm{E}+02$ & $3.75 \mathrm{E}+0 \mathrm{l}$ & $3.20 \mathrm{E}+02$ & $9.77 \mathrm{E}-01$ & $3.09 \mathrm{E}+02$ & $1.55 \mathrm{E}+02$ \\
\hline 21 & $4.08 \mathrm{E}+02$ & $7.66 \mathrm{E}+01$ & $3.12 \mathrm{E}+02$ & $3.78 \mathrm{E}+01$ & $3.22 \mathrm{E}+02$ & $1.06 \mathrm{E}+00$ & $3.09 \mathrm{E}+02$ & $1.55 \mathrm{E}+02$ \\
\hline 22 & $3.93 \mathrm{E}+02$ & $7.72 \mathrm{E}+01$ & $2.93 \mathrm{E}+02$ & $3.81 \mathrm{E}+01$ & $3.23 \mathrm{E}+02$ & $1.14 \mathrm{E}+00$ & $3.10 \mathrm{E}+02$ & $1.56 \mathrm{E}+02$ \\
\hline 23 & $3.80 \mathrm{E}+02$ & $7.78 \mathrm{E}+01$ & $2.76 \mathrm{E}+02$ & $3.84 \mathrm{E}+01$ & $3.25 \mathrm{E}+02$ & $1.23 \mathrm{E}+00$ & $3.11 \mathrm{E}+02$ & $1.56 \mathrm{E}+02$ \\
\hline 24 & $3.67 \mathrm{E}+02$ & $7.84 \mathrm{E}+01$ & $2.61 E+02$ & $3.86 \mathrm{E}+01$ & $3.26 \mathrm{E}+02$ & $1.31 \mathrm{E}+00$ & $3.12 \mathrm{E}+02$ & $1.57 \mathrm{E}+02$ \\
\hline 25 & $3.55 \mathrm{E}+02$ & $7.90 \mathrm{E}+01$ & $2.47 \mathrm{E}+02$ & $3.88 \mathrm{E}+01$ & $3.27 \mathrm{E}+02$ & $1.40 \mathrm{E}+00$ & $3.12 \mathrm{E}+02$ & $1.57 \mathrm{E}+02$ \\
\hline 26 & $3.44 \mathrm{E}+02$ & $7.95 \mathrm{E}+01$ & $2.35 \mathrm{E}+02$ & $3.91 \mathrm{E}+01$ & $3.28 \mathrm{E}+02$ & $1.48 \mathrm{E}+00$ & $3.13 E+02$ & $1.57 \mathrm{E}+02$ \\
\hline 27 & $3.23 \mathrm{E}+02$ & $8.00 \mathrm{E}+01$ & $2.24 \mathrm{E}+02$ & $3.93 \mathrm{E}+01$ & $3.28 \mathrm{E}+02$ & $1.56 \mathrm{E}+00$ & $3.13 \mathrm{E}+02$ & $1.58 \mathrm{E}+02$ \\
\hline 28 & $3.10 \mathrm{E}+02$ & $8.05 \mathrm{E}+01$ & $2.12 \mathrm{E}+02$ & $3.95 \mathrm{E}+01$ & $3.29 \mathrm{E}+02$ & $1.65 \mathrm{E}+00$ & $3.14 \mathrm{E}+02$ & $1.58 \mathrm{E}+02$ \\
\hline 29 & $2.99 \mathrm{E}+02$ & $8.09 \mathrm{E}+01$ & $2.01 \mathrm{E}+02$ & $3.97 \mathrm{E}+01$ & $3.29 \mathrm{E}+02$ & $1.74 \mathrm{E}+00$ & $3.14 E+02$ & $1.58 \mathrm{E}+02$ \\
\hline 30 & $2.90 \mathrm{E}+02$ & $8.14 \mathrm{E}+01$ & $1.91 \mathrm{E}+02$ & $3.99 \mathrm{E}+01$ & $3.27 \mathrm{E}+02$ & $1.77 \mathrm{E}+00$ & $3.16 \mathrm{E}+02$ & $9.89 \mathrm{E}+01$ \\
\hline 31 & $2.79 \mathrm{E}+02$ & $8.18 \mathrm{E}+01$ & $1.81 \mathrm{E}+02$ & $4.01 \mathrm{E}+01$ & $3.24 \mathrm{E}+02$ & $1.80 \mathrm{E}+00$ & $3.15 E+02$ & $9.85 \mathrm{E}+01$ \\
\hline 32 & $2.70 \mathrm{E}+02$ & $8.22 \mathrm{E}+01$ & $1.72 \mathrm{E}+02$ & $4.02 \mathrm{E}+01$ & $3.21 E+02$ & $1.84 \mathrm{E}+00$ & $3.14 \mathrm{E}+02$ & $9.81 \mathrm{E}+01$ \\
\hline 33 & $2.62 \mathrm{E}+02$ & $8.25 \mathrm{E}+01$ & $1.64 \mathrm{E}+02$ & $4.04 \mathrm{E}+01$ & $3.19 \mathrm{E}+02$ & $1.87 \mathrm{E}+00$ & $3.13 \bar{E}+02$ & $9.77 \mathrm{E}+01$ \\
\hline 34 & $2.55 \mathrm{E}+02$ & $8.29 \mathrm{E}+01$ & $1.56 \mathrm{E}+02$ & $4.05 \mathrm{E}+01$ & $3.16 \mathrm{E}+02$ & $1.90 \mathrm{E}+00$ & $3.11 \mathrm{E}+02$ & $9.73 \mathrm{E}+01$ \\
\hline 35 & $2.48 \mathrm{E}+02$ & $8.32 \mathrm{E}+01$ & $1.49 \mathrm{E}+02$ & $4.07 \mathrm{E}+01$ & $3.13 \mathrm{E}+02$ & $1.93 \mathrm{E}+00$ & $3.10 \mathrm{E}+02$ & $9.69 \mathrm{E}+01$ \\
\hline 36 & $2.42 \mathrm{E}+02$ & $8.36 \mathrm{E}+01$ & $1.42 \mathrm{E}+02$ & $4.08 \mathrm{E}+01$ & $3.11 \mathrm{E}+02$ & $1.97 \mathrm{E}+00$ & $3.09 \mathrm{E}+02$ & $9.65 \mathrm{E}+01$ \\
\hline 37 & $2.32 \mathrm{E}+02$ & $8.39 \mathrm{E}+01$ & $1.36 \mathrm{E}+02$ & $4.09 \mathrm{E}+01$ & $3.08 \mathrm{E}+02$ & $2.00 \mathrm{E}+00$ & $3.08 \mathrm{E}+02$ & $9.61 \mathrm{E}+01$ \\
\hline 38 & $2.26 \mathrm{E}+02$ & $8.42 \mathrm{E}+01$ & $1.30 \mathrm{E}+02$ & $4.11 \mathrm{E}+01$ & $3.06 \mathrm{E}+02$ & $2.03 \mathrm{E}+00$ & $3.06 \mathrm{E}+02$ & $9.57 \mathrm{E}+01$ \\
\hline
\end{tabular}




\begin{tabular}{|c|c|c|c|c|c|c|c|c|}
\hline 39 & $2.21 \mathrm{E}+02$ & $8.45 \mathrm{E}+01$ & $1.24 \mathrm{E}+02$ & $4.12 \mathrm{E}+01$ & $3.03 \mathrm{E}+02$ & $2.06 \mathrm{E}+00$ & $3.05 \mathrm{E}+02$ & 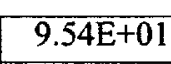 \\
\hline 40 & $2.16 \mathrm{E}+02$ & $8.48 \mathrm{E}+01$ & $1.19 \mathrm{E}+02$ & $4.13 \mathrm{E}+01$ & $3.01 \mathrm{E}+02$ & $2.09 \mathrm{E}+00$ & $3.04 \mathrm{E}+02$ & $9.50 \mathrm{E}$ \\
\hline 41 & $2.12 \mathrm{E}+02$ & $8.50 \mathrm{E}+01$ & $1.14 \mathrm{E}+02$ & $4.14 \mathrm{E}+01$ & $2.98 \mathrm{E}+02$ & $2.12 \mathrm{E}+00$ & $3.03 E+02$ & $.46 \mathrm{E}-$ \\
\hline 42 & $99 \mathrm{E}+02$ & $8.53 E+01$ & $1.10 \mathrm{E}+02$ & $4.15 \mathrm{E}+01$ & $2.98 \mathrm{E}+02$ & $2.21 E+00$ & $2.89 \mathrm{E}+02$ & $1.55 \mathrm{E}$ \\
\hline$\overline{43}$ & $1.94 \mathrm{E}+02$ & $8.55 \mathrm{E}+01$ & $1.05 E+02$ & $4.16 \mathrm{E}+01$ & $2.97 \mathrm{E}+02$ & $2.29 \mathrm{E}+00$ & $2.88 \mathrm{E}+02$ & $1.55 \mathrm{E}$ \\
\hline 44 & $1.90 \mathrm{E}+02$ & $8.58 \mathrm{E}+01$ & $9.95 E+01$ & $4.17 \mathrm{E}+01$ & $2.97 \mathrm{E}+02$ & $2.37 \mathrm{E}+00$ & $2.88 \mathrm{E}+02$ & $1.55 \mathrm{E}+$ \\
\hline 45 & $1.85 \mathrm{E}+02$ & $8.60 E+01$ & $9.50 \mathrm{E}+01$ & $4.18 \mathrm{E}+01$ & $2.96 \mathrm{E}+02$ & $2.46 \mathrm{E}+00$ & $2.88 \mathrm{E}+02$ & $1.55 \mathrm{E}+$ \\
\hline 46 & $1.82 \mathrm{E}+02$ & $8.62 \mathrm{E}+01$ & $9.07 \mathrm{E}+01$ & $4.19 \mathrm{E}+01$ & $2.96 \mathrm{E}+02$ & $2.54 \mathrm{E}+00$ & $2.88 \mathrm{E}+02$ & $1.55 \mathrm{E}+$ \\
\hline 47 & $1.80 \mathrm{E}+02$ & $8.64 \mathrm{E}+01$ & $8.68 \mathrm{E}+01$ & $4.20 \mathrm{E}+01$ & $2.95 \mathrm{E}+02$ & $2.63 \mathrm{E}+00$ & $2.87 \mathrm{E}+02$ & $1.55 \mathrm{E}+\mathrm{c}$ \\
\hline 48 & $1.76 \mathrm{E}+02$ & $8.66 E+01$ & $8.34 \mathrm{E}+01$ & $4.21 \mathrm{E}+01$ & $2.94 \mathrm{E}+02$ & $2.71 \mathrm{E}+00$ & $2.87 \mathrm{E}+02$ & $1.55 \mathrm{E}$ \\
\hline 49 & $1.74 \mathrm{E}+02$ & $8.68 \mathrm{E}+01$ & $8.02 \mathrm{E}+01$ & $4.22 \mathrm{E}+01$ & $2.93 \mathrm{E}+02$ & $2.79 \mathrm{E}+00$ & $2.87 \mathrm{E}+02$ & $1.55 \mathrm{E}+$ \\
\hline 50 & $1.72 \mathrm{E}+02$ & $8.70 \mathrm{E}+01$ & $7.75 \mathrm{E}+01$ & $4.23 \mathrm{E}+01$ & $2.93 \mathrm{E}+02$ & $2.88 \mathrm{E}+00$ & $2.86 \mathrm{E}+02$ & $1.55 \mathrm{E}+$ \\
\hline 51 & $1.70 \mathrm{E}+02$ & $8.72 \mathrm{E}+01$ & $7.51 \mathrm{E}+01$ & $4.23 \mathrm{E}+01$ & $2.92 \mathrm{E}+02$ & $2.96 \mathrm{E}+00$ & $2.86 \mathrm{E}+02$ & 1.551 \\
\hline 52 & $1.69 \mathrm{E}+02$ & $8.74 \mathrm{E}+01$ & $7.30 \mathrm{E}+01$ & $4.24 \mathrm{E}+01$ & $2.91 \mathrm{E}+02$ & $3.04 \mathrm{E}+00$ & $2.86 \mathrm{E}+02$ & $1.55 \mathrm{E}+$ \\
\hline 53 & $1.67 \mathrm{E}+02$ & $8.76 \mathrm{E}+01$ & $7.12 \mathrm{E}+01$ & $4.25 \mathrm{E}+01$ & $2.90 \mathrm{E}+02$ & $3.12 \mathrm{E}+00$ & $2.85 \mathrm{E}+02$ & $1.55 \mathrm{E}+$ \\
\hline 54 & $1.66 \mathrm{E}+02$ & $8.78 \mathrm{E}+01$ & $6.97 \mathrm{E}+01$ & $4.26 \mathrm{E}+01$ & $2.88 \mathrm{E}+02$ & $3.15 \mathrm{E}+00$ & $2.79 \mathrm{E}+02$ & $8.75 \mathrm{E}+$ \\
\hline 55 & $1.65 \mathrm{E}+02$ & $8.80 \mathrm{E}+01$ & $6.84 \mathrm{E}+01$ & $4.26 \mathrm{E}+01$ & $2.85 \mathrm{E}+02$ & $3.18 \mathrm{E}+00$ & $2.78 \mathrm{E}+02$ & $8.71 \mathrm{E}+$ \\
\hline 56 & $1.63 \mathrm{E}+02$ & $8.81 \mathrm{E}+01$ & $6.73 \mathrm{E}+01$ & $4.27 \mathrm{E}+01$ & $2.83 \mathrm{E}+02$ & $3.21 \mathrm{E}+00$ & $2.77 \mathrm{E}+02$ & $8.68 \mathrm{E}+$ \\
\hline 57 & $1.62 \mathrm{E}+02$ & $8.83 \mathrm{E}+01$ & $6.62 \mathrm{E}+01$ & $4.28 \mathrm{E}+01$ & $2.81 \mathrm{E}+02$ & $3.24 \mathrm{E}+00$ & $2.76 \mathrm{E}+02$ & $8.64 \mathrm{E}+$ \\
\hline 58 & $1.61 \mathrm{E}+02$ & $8.85 E+01$ & $6.53 \mathrm{E}+01$ & $4.28 \mathrm{E}+01$ & $2.78 \mathrm{E}+02$ & $3.27 \mathrm{E}+00$ & $2.74 \mathrm{E}+02$ & $8.61 \mathrm{E}+$ \\
\hline 59 & $1.60 \mathrm{E}+02$ & $8.87 \mathrm{E}+01$ & $6.45 \mathrm{E}+01$ & $4.29 \mathrm{E}+01$ & $2.76 \mathrm{E}+02$ & $3.29 \mathrm{E}+00$ & $2.73 E+02$ & $8.57 \mathrm{E}+$ \\
\hline 60 & $1.59 \mathrm{E}+02$ & $8.88 \mathrm{E}+01$ & $6.38 \mathrm{E}+01$ & $4.30 \mathrm{E}+01$ & $2.74 \mathrm{E}+02$ & $3.32 \mathrm{E}+00$ & $2.72 \mathrm{E}+02$ & $8.54 \mathrm{E}+$ \\
\hline 61 & $1.58 \mathrm{E}+02$ & $8.90 \mathrm{E}+01$ & $6.31 \mathrm{E}+01$ & $4.30 \mathrm{E}+01$ & $2.71 \mathrm{E}+02$ & $3.35 \mathrm{E}+00$ & $2.71 \mathrm{E}+02$ & $8.50 \mathrm{E}+$ \\
\hline 62 & $1.57 \mathrm{E}+02$ & $8.92 \mathrm{E}+01$ & $6.24 \mathrm{E}+01$ & $4.31 E+01$ & $2.69 \mathrm{E}+02$ & $3.37 \mathrm{E}+00$ & $2.70 \mathrm{E}+02$ & $8.47 \mathrm{E}+$ \\
\hline 63 & $1.56 \mathrm{E}+02$ & $8.93 \mathrm{E}+01$ & $6.18 \mathrm{E}+01$ & $4.32 \mathrm{E}+01$ & $2.67 \mathrm{E}+02$ & $3.40 \mathrm{E}+00$ & $2.69 \mathrm{E}+02$ & $8.43 \mathrm{E}+$ \\
\hline 64 & $1.55 \mathrm{E}+02$ & $8.95 \mathrm{E}+01$ & $6.12 \mathrm{E}+01$ & $4.32 \mathrm{E}+01$ & $2.65 \mathrm{E}+02$ & $3.43 \mathrm{E}+00$ & $2.68 \mathrm{E}+02$ & $8.40 \mathrm{E}+$ \\
\hline 65 & $1.54 \mathrm{E}+02$ & $8.96 \mathrm{E}+01$ & $6.06 \mathrm{E}+01$ & $4.33 \mathrm{E}+01$ & $2.63 \mathrm{E}+02$ & $3.45 \mathrm{E}+00$ & $2.67 \mathrm{E}+02$ & $8.36 \mathrm{E}+$ \\
\hline 66 & $1.53 \mathrm{E}+02$ & $8.98 \mathrm{E}+01$ & $6.01 \mathrm{E}+01$ & $4.34 \mathrm{E}+01$ & $2.62 \mathrm{E}+02$ & $3.53 \mathrm{E}+00$ & $2.54 \mathrm{E}+02$ & $1.46 \mathrm{E}+\mathrm{C}$ \\
\hline 67 & $1.52 \mathrm{E}+02$ & $8.99 \mathrm{E}+01$ & $5.96 \mathrm{E}+01$ & $4.34 \mathrm{E}+01$ & $2.61 E+02$ & $3.61 \mathrm{E}+00$ & $2.53 \mathrm{E}+02$ & $1.46 \mathrm{E}+\mathrm{C}$ \\
\hline 68 & $1.51 \mathrm{E}+02$ & $9.01 \mathrm{E}+01$ & $5.91 \mathrm{E}+01$ & $4.35 \mathrm{E}+01$ & $2.60 \mathrm{E}+02$ & $3.69 \mathrm{E}+00$ & $2.53 \mathrm{E}+02$ & $1.46 \mathrm{E}+\mathrm{c}$ \\
\hline 69 & $1.50 \mathrm{E}+02$ & $9.02 \mathrm{E}+01$ & $5.86 \mathrm{E}+01$ & $4.36 \mathrm{E}+01$ & $2.60 \mathrm{E}+02$ & $3.77 \mathrm{E}+00$ & $2.53 \mathrm{E}+02$ & $1.46 \mathrm{E}+$ \\
\hline 168 & $4.41 E+01$ & $9.04 \mathrm{E}+01$ & $4.59 \mathrm{E}+01$ & $4.86 E+01$ & $1.41 E+02$ & $1.05 E+01$ & $1.65 \mathrm{E}+02$ & $1.18 \mathrm{E}+$ \\
\hline 336 & $2.52 \mathrm{E}+01$ & $7.66 \mathrm{E}+01$ & $2.56 \mathrm{E}+01$ & $5.22 \mathrm{E}+01$ & $6.12 \mathrm{E}+01$ & $1.81 \mathrm{E}+01$ & $9.20 \mathrm{E}+01$ & $8.68 \mathrm{E}+$ \\
\hline 504 & $1.88 \mathrm{E}+01$ & $6.42 \mathrm{E}+01$ & $1.79 \mathrm{E}+01$ & $5.45 \mathrm{E}+01$ & $3.13 \mathrm{E}+01$ & $2.23 E+01$ & $4.28 \mathrm{E}+01$ & $5.96 \mathrm{E}+$ \\
\hline 720 & $1.38 \mathrm{E}+01$ & $5.08 \mathrm{E}+01$ & $1.21 \mathrm{E}+01$ & $5.65 \mathrm{E}+01$ & $1.63 \mathrm{E}+01$ & $2.46 \mathrm{E}+01$ & $2.21 \mathrm{E}+01$ & $4.20 \mathrm{E}+$ \\
\hline 2160 & $2.61 \mathrm{E}+00$ & $1.04 \mathrm{E}+01$ & $1.37 \mathrm{E}+00$ & $6.05 \mathrm{E}+01$ & $1.50 \mathrm{E}+00$ & $1.44 \mathrm{E}+01$ & $5.17 \mathrm{E}+00$ & $1.46 \mathrm{E}+$ \\
\hline 4320 & $2.51 \mathrm{E}-01$ & $9.57 \mathrm{E}-01$ & $6.86 \mathrm{E}+00$ & $5.72 \mathrm{E}+01$ & $2.56 \mathrm{E}-01$ & $3.46 \mathrm{E}+00$ & $6.43 \mathrm{E}-01$ & $2.32 \mathrm{E}+$ \\
\hline 4800 & $1.49 \mathrm{E}-01$ & $5.65 \mathrm{E}-01$ & $6.83 \mathrm{E}+00$ & $5.71 \mathrm{E}+01$ & $1.86 \mathrm{E}-01$ & $2.49 \mathrm{E}+00$ & $2.19 \mathrm{E}-01$ & $7.64 \mathrm{E}-1$ \\
\hline 8760 & 2.05E-03 & $7.51 \mathrm{E}-03$ & $6.66 \mathrm{E}+00$ & $5.56 \mathrm{E}+01$ & $1.55 \mathrm{E}-02$ & $1.68 \mathrm{E}-01$ & $6.86 \mathrm{E}-02$ & $2.26 \mathrm{E}-$ \\
\hline$\overline{B T}$ & $\begin{array}{l}2.4 \mathrm{E} 6 \\
\mathrm{pCi} / \mathrm{L}\end{array}$ & $\begin{array}{l}8.88 \mathrm{E}+4 \\
\mathrm{~Bq} / \mathrm{kg}\end{array}$ & $88.8 \mathrm{~Bq} / \mathrm{g}$ & & & & & \\
\hline To & $\begin{array}{l}5.9 \mathrm{E} 6 \\
\mathrm{pCi} / \mathrm{L}\end{array}$ & $\begin{array}{l}2.18 \mathrm{E}+5 \\
\mathrm{~Bq} / \mathrm{kg}\end{array}$ & $218.3 \mathrm{~Bq} / \mathrm{g}$ & & & & & \\
\hline & \multicolumn{2}{|c|}{ VEGETABLES } & \multicolumn{2}{|c|}{ WHEAT SEED } & \multicolumn{2}{|l|}{ MEAT } & \multicolumn{2}{|l|}{ MILK } \\
\hline \multirow[t]{2}{*}{ HOUR } & HTO & OBT & HTO & OBT & HTO & $\overline{\mathrm{BT}}$ & HTO & OBT \\
\hline & $(\mathrm{Bq} / \mathrm{g})$ & $(\mathrm{Bq} / \mathrm{g})$ & $(\mathrm{Bq} / \mathrm{g})$ & $(\mathrm{Bq} / \mathrm{g})$ & $(\mathrm{Bq} / \mathrm{g})$ & $(\mathrm{Bq} / \mathrm{g})$ & $(\mathrm{Bq} / \mathrm{g})$ & $(\mathrm{Bq} / \mathrm{g})$ \\
\hline ak HIO & & & & & & & & \\
\hline
\end{tabular}




\begin{tabular}{|l|l|l|l|l|l|l|l|l|}
\hline $1 / 3 / 5 / 6$ & $7.43 \mathrm{E}+03$ & $3.76 \mathrm{E}+01$ & $3.95 \mathrm{E}+03$ & $2.20 \mathrm{E}+01$ & $3.44 \mathrm{E}+02$ & $3.12 \mathrm{E}-01$ & $3.31 \mathrm{E}+02$ & $1.03 \mathrm{E}+02$ \\
\hline SUM & $7.47 \mathrm{E}+03$ & & $3.97 \mathrm{E}+03$ & & $3.45 \mathrm{E}+02$ & & $4.34 \mathrm{E}+02$ & \\
\hline Ci/g & $2.02 \mathrm{E}-07$ & & $1.07 \mathrm{E}-07$ & & $9.31 \mathrm{E}-09$ & & $1.17 \mathrm{E}-08$ & \\
\hline & & & & & & & & \\
\hline DIL HTO & $5.9 \mathrm{E}-09$ & & & & & & & \\
\hline (Ci/g) & & & & & & & & \\
\hline & & & & & & & & \\
\hline SUM 168 & $4.41 \mathrm{E}+01$ & $9.04 \mathrm{E}+01$ & $4.59 \mathrm{E}+01$ & $4.86 \mathrm{E}+01$ & $1.41 \mathrm{E}+02$ & $1.05 \mathrm{E}+01$ & $1.65 \mathrm{E}+02$ & $1.18 \mathrm{E}+02$ \\
\hline Ci/g & $1.35 \mathrm{E}+02$ & & $9.45 \mathrm{E}+01$ & & $1.52 \mathrm{E}+02$ & & $2.84 \mathrm{E}+02$ & \\
\hline & $3.64 \mathrm{E}-09$ & & $2.55 \mathrm{E}-09$ & & $4.10 \mathrm{E}-09$ & & $7.66 \mathrm{E}-09$ & \\
\hline DIL OBT & $2.4 \mathrm{E}-09$ & & & & & & & \\
\hline Ci/g & & & & & & & & \\
\hline & & & & & & & & \\
\hline \multicolumn{1}{|c|}{8760} & $2.05 \mathrm{E}-03$ & $7.51 \mathrm{E}-03$ & $6.66 \mathrm{E}+00$ & $5.56 \mathrm{E}+01$ & $1.55 \mathrm{E}-02$ & $1.68 \mathrm{E}-01$ & $6.86 \mathrm{E}-02$ & $2.26 \mathrm{E}-01$ \\
\hline SUM & $9.56 \mathrm{E}-03$ & & $6.23 \mathrm{E}+01$ & & $1.83 \mathrm{E}-01$ & & $2.95 \mathrm{E}-01$ & \\
\hline Ci/g & $2.58 \mathrm{E}-13$ & & $1.68 \mathrm{E}-09$ & & $4.95 \mathrm{E}-12$ & & $7.97 \mathrm{E}-12$ & \\
\hline & & & & & & & & \\
\hline DIL OBT & $2.4 \mathrm{E}-09$ & & & & & & & \\
\hline Ci/g & & & & & & & & \\
\hline
\end{tabular}




\begin{tabular}{|c|c|c|c|c|c|c|c|c|}
\hline & \multicolumn{6}{|c|}{ SRTC Release Scenario 1-RD-3, Average Met Conditions } & & \\
\hline & \multicolumn{4}{|c|}{ Concentration Values for $10 \mathrm{~km}$ from release } & & & & \\
\hline \multicolumn{9}{|c|}{ Only Wheat Seed is examined since this is the edible portion of the Wheat. The "WHEAT" category is } \\
\hline \multicolumn{7}{|c|}{ actually the unedible plant body and is not considered in the dose estimate. } & & \\
\hline & & & & & & & & \\
\hline & \multicolumn{2}{|c|}{ VEGETABLES } & \multicolumn{2}{|c|}{ WHEAT SEED } & \multicolumn{2}{|l|}{ MEAT } & \multicolumn{2}{|l|}{ MILK } \\
\hline \multirow[t]{2}{*}{ HOUR } & HTO & OBT & HTO & OBT & HTO & OBT & HTO & $\overline{\text { OBT }}$ \\
\hline & $(\mathrm{Bq} / \mathrm{g})$ & $(\mathrm{Bq} / \mathrm{g})$ & $(\mathrm{Bq} / \mathrm{g})$ & $(\mathrm{Bq} / \mathrm{g})$ & $(\mathrm{Bq} / \mathrm{g})$ & $(\mathrm{Bq} / \mathrm{g})$ & $(\overline{B q} / \mathrm{g})$ & $3 q$ \\
\hline & $182 \mathrm{~F}+0 \mathrm{l}$ & 2,10 & $2,20 \mathrm{C}+01$ & $00=5$ & & & & \\
\hline 1$]$ & $1.82 \mathrm{E}+02$ & 3. & $38 \mathrm{E}+01$ & 9.05E & 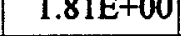 & & 01 & \\
\hline 2 & $3.37 \mathrm{E}+02$ & $2.20 \mathrm{E}+00$ & $1.51 \mathrm{E}+02$ & $6.02 \mathrm{E}-01$ & $1.08 \mathrm{E}+01$ & $2.69 \mathrm{E}-03$ & $1.36 \mathrm{E}+00$ & 6. \\
\hline 3 & $1.41 \mathrm{E}+02$ & $2.79 \mathrm{E}+00$ & $2.01 \mathrm{E}+02$ & $9.60 \mathrm{E}-01$ & $1.48 \mathrm{E}+01$ & $5.90 \mathrm{E}-03$ & $2.66 \mathrm{E}+00$ & 1.1 \\
\hline 4 & $8.09 \mathrm{E}+01$ & $3.04 \mathrm{E}+00$ & $2.07 \mathrm{E}+02$ & $1.21 \mathrm{E}+00$ & $1.67 \mathrm{E}+01$ & -03 & $3.89 \mathrm{E}+00$ & 1.7 \\
\hline 5 & $5.96 \mathrm{E}+01$ & $3.18 E+00$ & $1.92 \mathrm{E}+02$ & $1.38 \mathrm{E}+00$ & $1.78 \mathrm{E}+01$ & $1.35 \mathrm{E}-02$ & $5.00 \mathrm{E}+00$ & 2.2 \\
\hline 6 & $5.05 \mathrm{E}+01$ & $3.29 \mathrm{E}+00$ & $1.68 \mathrm{E}+02$ & $1.50 \mathrm{E}+00$ & $1.77 \mathrm{E}+01$ & $1.53 \mathrm{E}-02$ & $1.71 \mathrm{E}+01$ & $5.33 \mathrm{E}+00$ \\
\hline 7 & $4.56 \mathrm{E}+01$ & $3.37 \mathrm{E}+00$ & $1.43 \mathrm{E}+02$ & $1.59 \mathrm{E}+00$ & $1.75 \mathrm{E}+01$ & -02 & $1.71 \mathrm{E}+01$ & 5.3 \\
\hline 8 & $4.26 \mathrm{E}+01$ & $3.45 \mathrm{E}+00$ & $1.19 E+02$ & $1.66 \mathrm{E}+00$ & $1.74 \mathrm{E}+01$ & $1.91 \mathrm{E}-02$ & $1.70 \mathrm{E}+01$ & $5.30 \mathrm{E}+00$ \\
\hline 9 & $4.03 E+01$ & $3.52 \mathrm{E}+00$ & $9.83 \mathrm{E}+01$ & $1.72 \mathrm{E}+00$ & $1.73 \mathrm{E}+01$ & $2.09 \mathrm{E}-02$ & $1.69 \mathrm{E}+01$ & $5.28 \mathrm{E}+00$ \\
\hline 10 & $3.85 \mathrm{E}+01$ & $3.59 \mathrm{E}+00$ & $8.12 \mathrm{E}+01$ & $1.76 \mathrm{E}+00$ & $1.72 \mathrm{E}+01$ & -02 & $1.69 \mathrm{E}+01$ & 50 \\
\hline 11 & $3.69 \mathrm{E}+01$ & $3.65 \mathrm{E}+00$ & $6.74 \mathrm{E}+01$ & $1.80 \mathrm{E}+00$ & $1.70 \mathrm{E}+01$ & $2.46 \mathrm{E}-02$ & $1.68 \mathrm{E}+01$ & $5.24 \mathrm{E}+00$ \\
\hline 12 & $3.55 \mathrm{E}+01$ & $3.71 \mathrm{E}+00$ & $5.64 \mathrm{E}+01$ & $1.83 \mathrm{E}+00$ & $1.69 \mathrm{E}+01$ & $2.64 \mathrm{E}-02$ & $1.68 \mathrm{E}+01$ & $5.22 \mathrm{E}+00$ \\
\hline 13 & $3.43 \mathrm{E}+01$ & $3.77 \mathrm{E}+00$ & $4.78 \mathrm{E}+01$ & $1.86 \mathrm{E}+00$ & $1.68 \mathrm{E}+01$ & $2.82 \mathrm{E}-02$ & $1.67 \mathrm{E}+01$ & $5.20 \mathrm{E}+0$ \\
\hline 14 & $3.31 \mathrm{E}+01$ & $3.83 \mathrm{E}+00$ & $4.10 \mathrm{E}+01$ & $1.89 \mathrm{E}+00$ & $1.67 \mathrm{E}+01$ & $2.99 \mathrm{E}-02$ & $1.66 \mathrm{E}+01$ & 5.18 \\
\hline 15 & $3.20 \mathrm{E}+01$ & $3.88 \mathrm{E}+00$ & $3.58 \mathrm{E}+01$ & $1.92 \mathrm{E}+00$ & $1.65 \mathrm{E}+01$ & $3.17 \mathrm{E}-02$ & $1.66 \mathrm{E}+01$ & $5.16 \mathrm{E}+0 \mathrm{C}$ \\
\hline 16 & $3.10 \mathrm{E}+01$ & $3.94 \mathrm{E}+00$ & $3.17 \mathrm{E}+01$ & $1.94 \mathrm{E}+00$ & $1.64 \mathrm{E}+01$ & $3.34 \mathrm{E}-02$ & $1.65 \mathrm{E}+0 \mathrm{I}$ & $5.14 \mathrm{E}+0$ \\
\hline 17 & $3.00 \mathrm{E}+01$ & $3.99 \mathrm{E}+00$ & $2.85 \mathrm{E}+01$ & $1.96 \mathrm{E}+00$ & $1.63 \mathrm{E}+01$ & $3.52 \mathrm{E}-02$ & $1.65 \mathrm{E}+01$ & 8 \\
\hline 18 & $2.91 \mathrm{E}+01$ & $4.03 \mathrm{E}+00$ & $2.59 \mathrm{E}+01$ & $1.98 \mathrm{E}+00$ & $1.65 \mathrm{E}+01$ & $3.95 \mathrm{E}-02$ & $1.60 \mathrm{E}+01$ & $8.12 \mathrm{E}+0$ \\
\hline 19 & $2.82 \mathrm{E}+01$ & $4.08 \mathrm{E}+00$ & $2.38 \mathrm{E}+01$ & $2.01 \mathrm{E}+00$ & $1.66 \mathrm{E}+01$ & $4.39 \mathrm{E}-02$ & $1.60 \mathrm{E}+01$ & $8.16 \mathrm{E}+0$ \\
\hline 20 & $2.72 \mathrm{E}+01$ & $4.13 \mathrm{E}+00$ & $2.22 \mathrm{E}+01$ & $2.03 \mathrm{E}+00$ & $1.68 \mathrm{E}+01$ & $4.83 \mathrm{E}-02$ & $1.61 \mathrm{E}+01$ & 8.2 \\
\hline 21 & $2.64 \mathrm{E}+01$ & $4.17 \mathrm{E}+00$ & $2.07 \mathrm{E}+01$ & $2.04 \mathrm{E}+00$ & $1.69 \mathrm{E}+01$ & $5.27 \mathrm{E}-02$ & $1.62 \mathrm{E}+01$ & $8.24 \mathrm{E}+0$ \\
\hline 22 & $2.55 \mathrm{E}+01$ & $4.21 E+00$ & $1.95 \mathrm{E}+01$ & $2.06 \mathrm{E}+00$ & $1.70 \mathrm{E}+01$ & $5.72 \mathrm{E}-02$ & $1.62 \mathrm{E}+01$ & $8.27 \mathrm{E}+0$ \\
\hline 23 & $2.46 \mathrm{E}+01$ & $4.25 \mathrm{E}+00$ & $1.85 \mathrm{E}+01$ & $2.08 \mathrm{E}+00$ & $1.72 \mathrm{E}+01$ & $6.17 \mathrm{E}-02$ & $1.63 \mathrm{E}+01$ & 8.31 \\
\hline 24 & $2.38 \mathrm{E}+01$ & $4.29 \mathrm{E}+00$ & $1.76 \mathrm{E}+01$ & $2.10 \mathrm{E}+00$ & $1.73 \mathrm{E}+01$ & $6.63 \mathrm{E}-02$ & $1.64 \mathrm{E}+01$ & $8.34 \mathrm{E}+0$ \\
\hline 25 & $2.31 \mathrm{E}+01$ & $4.33 \mathrm{E}+00$ & $1.67 \mathrm{E}+01$ & $2.11 \mathrm{E}+00$ & $1.74 \mathrm{E}+01$ & $7.09 \mathrm{E}-02$ & $1.64 \mathrm{E}+01$ & $8.37 \mathrm{E}+0$ \\
\hline 26 & $2.23 \mathrm{E}+01$ & $4.36 \mathrm{E}+00$ & $1.60 \mathrm{E}+01$ & $2.13 E+00$ & $1.75 \mathrm{E}+01$ & $7.55 \mathrm{E}-02$ & $1.65 \mathrm{E}+01$ & $8.40 \mathrm{E}+0$ \\
\hline 27 & $2.16 \mathrm{E}+01$ & $4.40 \mathrm{E}+00$ & $1.53 \mathrm{E}+01$ & $2.15 \bar{E}+00$ & $1.75 \mathrm{E}+01$ & $8.01 \mathrm{E}-02$ & $1.65 E+01$ & $8.43 \mathrm{E}+0$ \\
\hline 28 & $2.08 \mathrm{E}+01$ & $4.43 E+00$ & $1.46 \mathrm{E}+01$ & $2.16 \mathrm{E}+00$ & $1.76 \mathrm{E}+01$ & $8.47 \mathrm{E}-02$ & $1.66 \mathrm{E}+01$ & $8.45 \mathrm{E}+0$ \\
\hline 29 & $2.01 \mathrm{E}+01$ & $4.46 \mathrm{E}+00$ & $1.40 \mathrm{E}+01$ & $2.17 \mathrm{E}+00$ & $1.77 \mathrm{E}+01$ & $8.94 \mathrm{E}-02$ & $1.66 \mathrm{E}+01$ & $8.48 \mathrm{E}+0$ \\
\hline 30 & $1.95 \mathrm{E}+01$ & $4.49 \mathrm{E}+00$ & $1.34 \mathrm{E}+01$ & $2.19 \mathrm{E}+00$ & $1.75 \mathrm{E}+01$ & $9.12 \mathrm{E}-02$ & $1.70 \mathrm{E}+01$ & $5.31 \mathrm{E}+0$ \\
\hline 31 & $1.89 \mathrm{E}+01$ & $4.52 \mathrm{E}+00$ & $1.28 \mathrm{E}+01$ & $2.20 \mathrm{E}+00$ & $1.74 \mathrm{E}+01$ & $9.30 \mathrm{E}-02$ & $1.69 \mathrm{E}+01$ & $5.28 \mathrm{E}+0$ \\
\hline 32 & $1.83 \mathrm{E}+01$ & $4.55 \mathrm{E}+00$ & $1.23 \mathrm{E}+01$ & $2.21 \mathrm{E}+00$ & $1.72 \mathrm{E}+01$ & $9.48 \mathrm{E}-02$ & $1.69 \mathrm{E}+01$ & $5.26 \mathrm{E}+0$ \\
\hline 33 & $1.77 \mathrm{E}+01$ & $4.57 \mathrm{E}+00$ & $1.18 \bar{E}+01$ & $2.22 \mathrm{E}+00$ & $1.71 \mathrm{E}+01$ & $9.65 \mathrm{E}-02$ & $1.68 \mathrm{E}+01$ & $5.24 \mathrm{E}+0$ \\
\hline 34 & $1.72 \mathrm{E}+01$ & $4.60 \mathrm{E}+00$ & $1.13 \mathrm{E}+01$ & $2.23 \mathrm{E}+00$ & $1.70 \mathrm{E}+01$ & $9.83 \mathrm{E}-02$ & $1.67 \mathrm{E}+01$ & $5.22 \mathrm{E}+0$ \\
\hline 35 & $1.67 \mathrm{E}+01$ & $4.62 \mathrm{E}+00$ & $1.08 \mathrm{E}+01$ & $2.25 \mathrm{E}+00$ & $1.68 \mathrm{E}+01$ & $1.00 \mathrm{E}-01$ & $1.66 \mathrm{E}+01$ & $5.20 \mathrm{E}+0$ \\
\hline 36 & $1.62 \mathrm{E}+01$ & $4.65 E+00$ & $1.04 \mathrm{E}+01$ & $2.26 \mathrm{E}+00$ & $1.67 \mathrm{E}+01$ & $1.02 \mathrm{E}-01$ & $1.66 \mathrm{E}+01$ & $5.18 \mathrm{E}+0$ \\
\hline 37 & $1.57 \mathrm{E}+01$ & $4.67 \mathrm{E}+00$ & $9.98 \mathrm{E}+00$ & $2.27 \mathrm{E}+00$ & $1.66 \mathrm{E}+01$ & $1.04 \mathrm{E}-01$ & $1.65 \mathrm{E}+01$ & $5.16 \mathrm{E}+0$ \\
\hline 38 & $1.53 \mathrm{E}+01$ & $4.69 \mathrm{E}+00$ & $9.59 \mathrm{E}+00$ & $2.28 \mathrm{E}+00$ & $1.64 \mathrm{E}+01$ & $1.05 \mathrm{E}-01$ & $1.64 \mathrm{E}+01$ & $5.14 \mathrm{E}+0$ \\
\hline
\end{tabular}




\begin{tabular}{|c|c|c|c|c|c|c|c|c|}
\hline 39 & $1.50 \mathrm{E}+01$ & $4.71 \mathrm{E}+00$ & $9.22 \mathrm{E}+00$ & $2.28 \mathrm{E}+00$ & $1.63 \mathrm{E}+01$ & $1.07 \mathrm{E}-01$ & $1.64 \mathrm{E}+01$ & $5.12 \mathrm{E}+\mathrm{C}$ \\
\hline 40 & $1.47 \mathrm{E}+01$ & $.73 \mathrm{E}+00$ & $8.87 \mathrm{E}+00$ & $2.29 \mathrm{E}+00$ & $1.62 \mathrm{E}+01$ & $1.09 \mathrm{E}-01$ & $1.63 \mathrm{E}+01$ & $5.10 \mathrm{E}+\mathrm{C}$ \\
\hline 41 & $1.43 \mathrm{E}+01$ & $4.75 \mathrm{E}+00$ & $8.56 \mathrm{E}+00$ & $2.30 \mathrm{E}+00$ & $1.60 \mathrm{E}+01$ & $1.10 \mathrm{E}-01$ & $1.63 \mathrm{E}+01$ & $5.08 \mathrm{E}+0$ \\
\hline 42 & $1.40 \mathrm{E}+01$ & $4.77 \mathrm{E}+00$ & $.26 \mathrm{E}+00$ & $2.31 \mathrm{E}+00$ & $1.60 \mathrm{E}+01$ & $1.15 \mathrm{E}-01$ & $1.55 \mathrm{E}+01$ & $.50 \mathrm{E}+$ \\
\hline$\overline{43}$ & $1.37 \mathrm{E}+01$ & $4.79 \mathrm{E}+00$ & $7.98 \mathrm{E}+00$ & $2.32 \mathrm{E}+00$ & $1.60 \mathrm{E}+01$ & $1.20 \mathrm{E}-01$ & $1.55 \mathrm{E}+01$ & $8.51 \mathrm{E}+0$ \\
\hline$\overline{44}$ & $1.34 \mathrm{E}+01$ & $4.81 \mathrm{E}+00$ & $7.72 \mathrm{E}+00$ & $2.33 \mathrm{E}+00$ & $1.60 \mathrm{E}+01$ & $1.24 \mathrm{E}-01$ & $1.55 \mathrm{E}+01$ & $8.51 \mathrm{E}+\mathrm{C}$ \\
\hline 45 & $1.32 \mathrm{E}+01$ & $4.83 E+00$ & $7.47 \mathrm{E}+00$ & $2.33 \mathrm{E}+00$ & $1.60 \mathrm{E}+01$ & $1.29 \mathrm{E}-01$ & $1.55 \mathrm{E}+01$ & $3.51 \mathrm{E}+$ \\
\hline 46 & $1.29 \mathrm{E}+01$ & $4.84 \mathrm{E}+00$ & $7.24 \mathrm{E}+00$ & $2.34 \mathrm{E}+00$ & $1.60 \mathrm{E}+01$ & $1.33 \mathrm{E}-01$ & $1.55 \mathrm{E}+01$ & $8.52 \mathrm{E}+0$ \\
\hline 47 & $1.27 \mathrm{E}+01$ & $4.86 \mathrm{E}+00$ & $7.02 \mathrm{E}+00$ & $2.35 E+00$ & $1.60 \mathrm{E}+01$ & $1.38 \mathrm{E}-01$ & $1.55 \mathrm{E}+01$ & $8.52 \mathrm{E}+0$ \\
\hline 48 & $1.24 \mathrm{E}+01$ & $4.88 \mathrm{E}+00$ & $6.81 \mathrm{E}+00$ & $2.36 \mathrm{E}+00$ & $1.60 \mathrm{E}+01$ & $1.43 \mathrm{E}-01$ & $1.55 \mathrm{E}+01$ & $8.52 \mathrm{E}+\mathrm{C}$ \\
\hline$\overline{49}$ & $1.22 \mathrm{E}+01$ & $4.89 \mathrm{E}+00$ & $6.61 \mathrm{E}+00$ & $2.36 \mathrm{E}+00$ & $1.60 \mathrm{E}+01$ & $1.47 \mathrm{E}-01$ & $1.55 \mathrm{E}+01$ & $8.53 \mathrm{E}+\mathrm{C}$ \\
\hline 50 & $1.20 \mathrm{E}+01$ & $4.91 \mathrm{E}+00$ & $6.42 \mathrm{E}+00$ & $2.37 \mathrm{E}+00$ & $1.60 \mathrm{E}+01$ & $1.52 \mathrm{E}-01$ & $1.55 \mathrm{E}+01$ & $8.53 \mathrm{E}+\mathrm{C}$ \\
\hline 51 & $1.18 \mathrm{E}+01$ & $4.92 \mathrm{E}+00$ & $6.25 \mathrm{E}+00$ & $2.38 \mathrm{E}+00$ & $1.60 \mathrm{E}+01$ & $1.57 \mathrm{E}-01$ & $1.55 \mathrm{E}+01$ & $8.53 \mathrm{E}+$ \\
\hline 52 & $1.17 \mathrm{E}+01$ & $4.94 E+00$ & $6.08 \mathrm{E}+00$ & $2.38 \mathrm{E}+00$ & $1.60 \mathrm{E}+01$ & $1.61 \mathrm{E}-01$ & $1.55 \mathrm{E}+01$ & $8.53 \mathrm{E}+$ \\
\hline 53 & $1.16 \mathrm{E}+01$ & $4.95 \mathrm{E}+00$ & $5.94 \mathrm{E}+00$ & $2.39 \mathrm{E}+00$ & $1.60 \mathrm{E}+01$ & $1.66 \mathrm{E}-01$ & $1.55 \mathrm{E}+01$ & $8.53 \mathrm{E}+\mathrm{C}$ \\
\hline 54 & $1.14 \mathrm{E}+01$ & $4.97 \mathrm{E}+00$ & $5.80 \mathrm{E}+00$ & $2.39 \mathrm{E}+00$ & $1.58 \mathrm{E}+01$ & $1.68 \mathrm{E}-01$ & $1.53 \mathrm{E}+01$ & $4.81 \mathrm{E}+\mathrm{C}$ \\
\hline 55 & $1.13 \mathrm{E}+01$ & $4.98 E+00$ & $5.68 \mathrm{E}+00$ & $2.40 \mathrm{E}+00$ & $1.57 \mathrm{E}+01$ & $1.69 \mathrm{E}-01$ & $1.53 \mathrm{E}+01$ & $4.79 \mathrm{E}+0$ \\
\hline 56 & $1.12 \mathrm{E}+01$ & $4.99 \mathrm{E}+00$ & $5.57 \mathrm{E}+00$ & $2.41 \mathrm{E}+00$ & $1.56 \mathrm{E}+01$ & 1.71E-01 & $1.52 \mathrm{E}+01$ & $4.77 \mathrm{E}+0$ \\
\hline 57 & $1.11 \mathrm{E}+01$ & $5.01 \mathrm{E}+00$ & $5.47 \mathrm{E}+00$ & $2.41 \mathrm{E}+00$ & $1.54 \mathrm{E}+01$ & $1.72 \mathrm{E}-01$ & $1.51 \mathrm{E}+01$ & $4.75 \mathrm{E}+\mathrm{C}$ \\
\hline 58 & $1.10 \mathrm{E}+01$ & $5.02 \mathrm{E}+00$ & $5.38 \mathrm{E}+00$ & $2.42 \mathrm{E}+00$ & $1.53 \mathrm{E}+01$ & $1.74 \mathrm{E}-01$ & $1.51 \mathrm{E}+01$ & $4.73 \mathrm{E}+0$ \\
\hline 59 & $1.09 \mathrm{E}+01$ & $5.03 \mathrm{E}+00$ & $5.30 \mathrm{E}+00$ & $2.42 E+00$ & $1.52 \mathrm{E}+01$ & $1.75 \mathrm{E}-01$ & $1.50 \mathrm{E}+0 \mathrm{I}$ & $\overline{4.71 \mathrm{E}+0}$ \\
\hline 60 & $1.08 \mathrm{E}+01$ & $5.05 \mathrm{E}+00$ & $5.22 \mathrm{E}+00$ & $2.43 \mathrm{E}+00$ & $1.51 \mathrm{E}+01$ & $1.77 \mathrm{E}-01$ & $1.50 \mathrm{E}+01$ & $\overline{4.69}$ \\
\hline 61 & $1.07 \mathrm{E}+01$ & $5.06 \mathrm{E}+00$ & $5.14 \mathrm{E}+00$ & $2.44 \mathrm{E}+00$ & $1.49 \mathrm{E}+01$ & $1.78 \mathrm{E}-01$ & $1.49 \mathrm{E}+01$ & $4.67 \mathrm{E}+\mathrm{C}$ \\
\hline 62 & $1.06 \mathrm{E}+01$ & $5.07 \mathrm{E}+00$ & $5.07 \mathrm{E}+00$ & $2.44 E+00$ & $1.48 \mathrm{E}+01$ & $1.80 \mathrm{E}-01$ & $1.48 \mathrm{E}+01$ & $4.65 \mathrm{E}+0$ \\
\hline 63 & $1.05 \mathrm{E}+01$ & $5.08 \mathrm{E}+00$ & $5.01 \mathrm{E}+00$ & $2.45 \mathrm{E}+00$ & $1.47 \mathrm{E}+01$ & $1.81 \mathrm{E}-01$ & $1.48 \mathrm{E}+01$ & $4.63 \mathrm{E}+$ \\
\hline 64 & $1.04 \mathrm{E}+01$ & $5.10 \mathrm{E}+00$ & $4.94 \mathrm{E}+00$ & $2.45 \mathrm{E}+00$ & $1.46 \mathrm{E}+01$ & $1.83 \mathrm{E}-01$ & $1.47 \mathrm{E}+01$ & $4.62 \mathrm{E}+\mathrm{C}$ \\
\hline 65 & $1.03 \mathrm{E}+01$ & $5.11 \mathrm{E}+00$ & $4.88 E+00$ & $2.46 \mathrm{E}+00$ & $1.44 \mathrm{E}+01$ & $1.84 \mathrm{E}-01$ & $1.47 \mathrm{E}+01$ & $4.60 \mathrm{E}+0$ \\
\hline 66 & $1.03 \mathrm{E}+01$ & $5.12 \mathrm{E}+00$ & $4.82 \mathrm{E}+00$ & $2.46 \mathrm{E}+00$ & $1.44 \mathrm{E}+01$ & $1.89 \mathrm{E}-01$ & $1.40 \mathrm{E}+01$ & $8.20 \mathrm{E}+$ \\
\hline 67 & $1.02 \mathrm{E}+01$ & $5.13 \mathrm{E}+00$ & $4.77 \mathrm{E}+00$ & $2.47 \mathrm{E}+00$ & $1.44 \mathrm{E}+01$ & $1.93 \mathrm{E}-01$ & $1.40 \mathrm{E}+01$ & $8.20 \mathrm{E}+\mathrm{C}$ \\
\hline 68 & $1.01 E+01$ & $5.14 E+00$ & $4.71 \mathrm{E}+00$ & $2.47 E+00$ & $1.44 \mathrm{E}+01$ & $1.98 \mathrm{E}-01$ & $1.40 \mathrm{E}+01$ & $8.19 \mathrm{E}+\mathrm{C}$ \\
\hline 69 & $1.00 \mathrm{E}+01$ & $5.15 \mathrm{E}+00$ & $4.66 \mathrm{E}+00$ & $2.48 \mathrm{E}+00$ & $1.44 \mathrm{E}+01$ & $2.02 \mathrm{E}-01$ & $1.39 \mathrm{E}+01$ & $8.19 \mathrm{E}+0$ \\
\hline 168 & $2.49 \mathrm{E}+00$ & $5.23 \mathrm{E}+00$ & $2.63 E+00$ & $2.81 E+00$ & $8.01 \mathrm{E}+00$ & $5.93 \mathrm{E}-01$ & $9.39 \mathrm{E}+00$ & $6.79 \mathrm{E}+\mathrm{C}$ \\
\hline 336 & $1.40 \mathrm{E}+00$ & $4.43 \mathrm{E}+00$ & $1.45 \mathrm{E}+00$ & $3.02 \mathrm{E}+00$ & $3.49 \mathrm{E}+00$ & $1.03 \mathrm{E}+00$ & $5.23 \mathrm{E}+00$ & $5.00 \mathrm{E}+\mathrm{C}$ \\
\hline 504 & $1.05 \mathrm{E}+00$ & $3.70 \mathrm{E}+00$ & $1.01 \mathrm{E}+00$ & $3.15 \mathrm{E}+00$ & $1.78 \mathrm{E}+00$ & $1.28 \mathrm{E}+00$ & $2.44 \mathrm{E}+00$ & $3.44 \mathrm{E}+\mathrm{C}$ \\
\hline 720 & $7.65 \mathrm{E}-01$ & $2.92 \mathrm{E}+00$ & $6.80 \mathrm{E}-01$ & $3.26 \mathrm{E}+00$ & $9.30 \mathrm{E}-01$ & $1.41 \mathrm{E}+00$ & $1.26 \mathrm{E}+00$ & $2.42 \mathrm{E}+\mathrm{C}$ \\
\hline 2160 & $1.44 \mathrm{E}-01$ & $5.89 \mathrm{E}-01$ & $7.70 \mathrm{E}-02$ & $3.49 \mathrm{E}+00$ & $8.46 \mathrm{E}-02$ & $8.28 \mathrm{E}-01$ & $2.95 \mathrm{E}-01$ & $8.40 \mathrm{E}-\mathrm{C}$ \\
\hline 4320 & $1.39 \mathrm{E}-02$ & $5.39 \mathrm{E}-02$ & $3.87 \mathrm{E}-01$ & $3.30 \mathrm{E}+00$ & $1.43 \mathrm{E}-02$ & $1.98 \mathrm{E}-01$ & $3.62 \mathrm{E}-02$ & $1.32 \mathrm{E}-\mathrm{C}$ \\
\hline 4800 & $8.25 \mathrm{E}-03$ & $3.18 \mathrm{E}-02$ & $3.85 \mathrm{E}-01$ & $3.29 \mathrm{E}+00$ & $1.04 \mathrm{E}-02$ & $1.43 \mathrm{E}-01$ & $1.22 \mathrm{E}-02$ & $4.35 \mathrm{E}-\mathrm{C}$ \\
\hline 8760 & $1.13 \mathrm{E}-04$ & $4.18 \mathrm{E}-04$ & $3.76 \mathrm{E}-01$ & $3.21 \mathrm{E}+00$ & $8.58 \mathrm{E}-04$ & $9.56 \mathrm{E}-03$ & $3.82 \mathrm{E}-03$ & $1.28 \mathrm{E}-($ \\
\hline BT & $\begin{array}{l}2.4 \mathrm{E} 6 \\
\mathrm{pCi} / \mathrm{L}\end{array}$ & $\begin{array}{l}8 E+4 \\
k g\end{array}$ & $8 \mathrm{~Bq} / \mathrm{g}$ & & & & & \\
\hline $\mathbf{0}$ & $\begin{array}{l}5.9 \mathrm{E} 6 \\
\mathrm{pCi} / \mathrm{L}\end{array}$ & $\begin{array}{l}2.18 \mathrm{E}+5 \\
\mathrm{~Bq} / \mathrm{kg} \\
\end{array}$ & $\sqrt{9}$ & & & & & \\
\hline & & & & & & & & \\
\hline & \multicolumn{2}{|c|}{ VEGETABLES } & \multicolumn{2}{|c|}{ WHEAT SEED } & \multicolumn{2}{|l|}{ MEAT } & \multicolumn{2}{|l|}{ MILK } \\
\hline \multirow[t]{2}{*}{ HOUR } & & OBT & 0 & OBT & TO & $B 1$ & TO & BOT \\
\hline & $(\mathrm{Bq} / \mathrm{g})$ & $(\mathrm{Bq} / \mathrm{g})$ & $(\overline{B q} / \mathrm{g})$ & $(\mathrm{Bq} / \mathrm{g})$ & $(\mathrm{Bq} / \mathrm{g})$ & $(\mathrm{Bq} / \mathrm{g})$ & $(\mathrm{Bq} / \mathrm{g})$ & $(\mathrm{Bq} / \mathrm{g})$ \\
\hline
\end{tabular}




\begin{tabular}{|c|c|c|c|c|c|c|c|c|}
\hline Peak HTO & & & & & & & & \\
\hline $2 / 4 / 6 / 6$ & $3.37 \mathrm{E}+02$ & $2.20 \mathrm{E}+00$ & $2.07 \mathrm{E}+02$ & $1.21 \mathrm{E}+00$ & $1.77 \mathrm{E}+01$ & $1.53 \mathrm{E}-02$ & $1.71 \mathrm{E}+01$ & $5.33 \mathrm{E}+00$ \\
\hline SUM & $3.39 \mathrm{E}+02$ & & $2.09 \mathrm{E}+02$ & & $1.77 \mathrm{E}+01$ & & $2.25 \mathrm{E}+01$ & \\
\hline $\mathrm{Ci} / \mathrm{g}$ & $9.16 \mathrm{E}-09$ & & $5.64 \mathrm{E}-09$ & & $4.78 \mathrm{E}-10$ & & $6.07 \mathrm{E}-10$ & \\
\hline & & & & & & & & \\
\hline DIL HTO & $5.9 \mathrm{E}-09$ & & & & & & & \\
\hline$(\mathrm{Ci} / \mathrm{g})$ & & & & & & & & \\
\hline & & & & & & & & \\
\hline 168 & $2.49 \mathrm{E}+00$ & $5.23 \mathrm{E}+00$ & $2.63 \mathrm{E}+00$ & $2.81 E+00$ & $8.01 \mathrm{E}+00$ & $5.93 \mathrm{E}-01$ & $9.39 \mathrm{E}+00$ & $6.79 \mathrm{E}+00$ \\
\hline SUM & $7.73 \mathrm{E}+00$ & & $5.44 E+00$ & & $8.61 \mathrm{E}+00$ & & $1.62 \mathrm{E}+01$ & \\
\hline $\mathrm{Ci} / \mathrm{g}$ & $2.09 \mathrm{E}-10$ & & $1.47 \mathrm{E}-10$ & & $2.33 \mathrm{E}-10$ & & $4.37 \mathrm{E}-10$ & \\
\hline & & & & & & & & \\
\hline DIL OBT & $2.4 \mathrm{E}-09$ & & & & & & & \\
\hline $\mathrm{Ci} / \mathrm{g}$ & & & & & & & & \\
\hline & & & & & & & & \\
\hline 8760 & $1.13 \mathrm{E}-04$ & $4.18 \mathrm{E}-04$ & $3.76 \mathrm{E}-01$ & $3.21 \mathrm{E}+00$ & $8.58 \mathrm{E}-04$ & $9.56 \mathrm{E}-03$ & $3.82 \mathrm{E}-03$ & $1.28 \mathrm{E}-02$ \\
\hline SUM & $5.31 \mathrm{E}-04$ & & $3.58 \mathrm{E}+00$ & & $1.04 \mathrm{E}-02$ & & $1.67 \mathrm{E}-02$ & \\
\hline $\mathrm{Ci} / \mathrm{g}$ & $1.44 \mathrm{E}-14$ & & $9.68 \mathrm{E}-11$ & & $2.82 \mathrm{E}-13$ & & $4.50 \mathrm{E}-13$ & \\
\hline DIL OBT & & & & & & & & \\
\hline DIL OBT & 2.4E-09 & & & & & & & \\
\hline $\mathrm{Ci} / \mathrm{g}$ & & & & & & & & \\
\hline & & & & & & & & \\
\hline
\end{tabular}




\begin{tabular}{|c|c|c|c|c|c|c|c|c|}
\hline & \multicolumn{6}{|c|}{ SRTC Release Scenario 1-RD-3, Average Met Conditions } & & \\
\hline & \multicolumn{4}{|c|}{ Concentration Values for $50 \mathrm{~km}$ from release } & & & & \\
\hline \multicolumn{9}{|c|}{ Only Wheat Seed is examined since this is the edible portion of the Wheat. The "WHEAT" category is } \\
\hline \multicolumn{9}{|c|}{ actually the unedible plant body and is not considered in the dose estimate. } \\
\hline & & & & & & & & \\
\hline & \multicolumn{2}{|c|}{ VEGETABLES } & \multicolumn{2}{|c|}{ WHEAT SEED } & \multicolumn{2}{|l|}{ MEAT } & \multicolumn{2}{|l|}{ MILK } \\
\hline \multirow[t]{2}{*}{ HOUR } & HTO & OBT & HTO & OBT & HTO & $\overline{\mathrm{OBT}}$ & HTO & OBT \\
\hline & $(\mathrm{Bq} / \mathrm{g})$ & $(\mathrm{Bq} / \mathrm{g})$ & $(\mathrm{Bq} / \mathrm{g})$ & $(\mathrm{Bq} / \mathrm{g})$ & $(\mathrm{Bq} / \mathrm{g})$ & $(\mathrm{Bq} / \mathrm{g})$ & $(\mathrm{Bq} / \mathrm{g})$ & $(\mathrm{Bq} / \mathrm{g})$ \\
\hline & & & & & & & & \\
\hline 1 & $1.45 \mathrm{E}+01$ & $0.00 \mathrm{E}+00$ & $0.00 \mathrm{E}+00$ & $0.00 \mathrm{E}+00$ & $1.37 \mathrm{E}-02$ & $1.47 \mathrm{E}-06$ & $1.66 \mathrm{E}-03$ & $5.16 \mathrm{E}-04$ \\
\hline 2 & $2.15 \mathrm{E}+01$ & $2.59 \mathrm{E}-02$ & $1.67 \mathrm{E}+00$ & $6.35 \mathrm{E}-03$ & $1.42 \mathrm{E}-01$ & $3.00 \mathrm{E}-05$ & $1.67 \mathrm{E}-02$ & $7.00 \mathrm{E}-03$ \\
\hline 3 & $1.85 \mathrm{E}+01$ & $6.44 \mathrm{E}-02$ & $4.30 \mathrm{E}+00$ & $1.82 \mathrm{E}-02$ & $3.36 \mathrm{E}-01$ & $1.01 \mathrm{E}-04$ & 4.77E-02 & $2.07 \mathrm{E}-02$ \\
\hline 4 & $2.43 \mathrm{E}+01$ & $9.74 \mathrm{E}-02$ & $6.52 \mathrm{E}+00$ & $3.15 \mathrm{E}-02$ & $5.27 \mathrm{E}-01$ & $2.14 \mathrm{E}-04$ & $8.98 \mathrm{E}-02$ & $3.95 \mathrm{E}-02$ \\
\hline 5 & $2.29 \mathrm{E}+01$ & $1.68 \mathrm{E}-01$ & $1.10 \mathrm{E}+01$ & $5.56 \mathrm{E}-02$ & $8.91 \mathrm{E}-01$ & $4.06 \mathrm{E}-04$ & $1.54 \mathrm{E}-01$ & $6.83 \mathrm{E}-02$ \\
\hline 6 & $7.21 \mathrm{E}+01$ & $5.98 \mathrm{E}-01$ & $4.03 \mathrm{E}+01$ & $1.80 \mathrm{E}-01$ & $1.10 \mathrm{E}+00$ & $5.24 \mathrm{E}-04$ & $1.07 \mathrm{E}+00$ & $3.33 \mathrm{E}-01$ \\
\hline 7 & $3.70 \mathrm{E}+01$ & $7.80 \mathrm{E}-01$ & $5.42 \mathrm{E}+01$ & $2.77 \mathrm{E}-01$ & $1.13 \mathrm{E}+00$ & $6.45 \mathrm{E}-04$ & $1.08 \mathrm{E}+00$ & $3.37 \mathrm{E}-01$ \\
\hline 8 & $2.01 E+01$ & $8.45 \mathrm{E}-01$ & $5.57 \mathrm{E}+01$ & $3.43 \mathrm{E}-01$ & $1.13 \mathrm{E}+00$ & $7.66 \mathrm{E}-04$ & $1.09 \mathrm{E}+00$ & $3.38 \mathrm{E}-01$ \\
\hline 9 & $1.51 \mathrm{E}+01$ & $8.80 \mathrm{E}-01$ & $5.13 \mathrm{E}+01$ & $3.89 \mathrm{E}-01$ & $1.13 \mathrm{E}+00$ & $8.87 \mathrm{E}-04$ & $1.09 \mathrm{E}+00$ & $3.39 \mathrm{E}-01$ \\
\hline 10 & $1.34 \mathrm{E}+01$ & $9.06 \mathrm{E}-01$ & $4.48 \mathrm{E}+01$ & $4.21 \mathrm{E}-01$ & $1.12 \mathrm{E}+00$ & $1.01 \mathrm{E}-03$ & $1.09 \mathrm{E}+00$ & $3.39 \mathrm{E}-01$ \\
\hline 11 & $1.25 \mathrm{E}+01$ & $9.29 \mathrm{E}-01$ & $3.79 \mathrm{E}+01$ & $4.44 \mathrm{E}-01$ & $1.12 \mathrm{E}+00$ & $1.13 \mathrm{E}-03$ & $1.09 \mathrm{E}+00$ & $3.39 \mathrm{E}-01$ \\
\hline 12 & $1.20 \mathrm{E}+01$ & $9.50 \mathrm{E}-01$ & $3.16 \mathrm{E}+01$ & $4.63 E-01$ & $1.12 \mathrm{E}+00$ & $1.25 \mathrm{E}-03$ & $1.09 \mathrm{E}+00$ & $3.39 \mathrm{E}-01$ \\
\hline 13 & $1.15 \mathrm{E}+01$ & $9.70 \mathrm{E}-01$ & $2.61 \mathrm{E}+01$ & $4.77 \mathrm{E}-01$ & $1.11 \mathrm{E}+00$ & $1.37 \mathrm{E}-03$ & $1.09 \mathrm{E}+00$ & $3.38 \mathrm{E}-01$ \\
\hline 14 & $1.12 \mathrm{E}+01$ & $9.89 \mathrm{E}-01$ & $2.17 \mathrm{E}+01$ & $4.89 \mathrm{E}-01$ & $1.11 \mathrm{E}+00$ & $1.48 \mathrm{E}-03$ & $1.09 \mathrm{E}+00$ & $3.38 \mathrm{E}-01$ \\
\hline-15 & $1.08 \mathrm{E}+01$ & $1.01 \mathrm{E}+00$ & $1.81 \mathrm{E}+01$ & $5.00 \mathrm{E}-01$ & $1.11 \mathrm{E}+00$ & $1.60 \mathrm{E}-03$ & $1.08 \mathrm{E}+00$ & $3.38 \mathrm{E}-01$ \\
\hline 16 & $1.05 \mathrm{E}+01$ & $1.03 \mathrm{E}+00$ & $1.53 \mathrm{E}+01$ & $5.09 \mathrm{E}-01$ & $1.10 \mathrm{E}+00$ & $1.72 \mathrm{E}-03$ & $1.08 \mathrm{E}+00$ & $3.37 \mathrm{E}-01$ \\
\hline 17 & $1.03 \mathrm{E}+01$ & $1.04 \mathrm{E}+00$ & $1.31 \mathrm{E}+01$ & $5.18 \mathrm{E}-01$ & $1.10 \mathrm{E}+00$ & $1.84 \mathrm{E}-03$ & $1.08 \mathrm{E}+00$ & $3.37 \mathrm{E}-01$ \\
\hline 18 & $1.00 \mathrm{E}+01$ & $1.06 \mathrm{E}+00$ & $1.15 \mathrm{E}+01$ & $5.26 \mathrm{E}-01$ & $1.20 \mathrm{E}+00$ & $2.65 \mathrm{E}-03$ & $1.16 \mathrm{E}+00$ & $1.19 \mathrm{E}+00$ \\
\hline 19 & $9.74 \mathrm{E}+00$ & $1.08 \mathrm{E}+00$ & $1.01 \mathrm{E}+01$ & $5.34 \mathrm{E}-01$ & $1.29 \mathrm{E}+00$ & $3.48 \mathrm{E}-03$ & $1.20 \mathrm{E}+00$ & $1.21 \mathrm{E}+00$ \\
\hline 20 & $9.48 \mathrm{E}+00$ & $1.09 \mathrm{E}+00$ & $9.12 \mathrm{E}+00$ & $5.41 \mathrm{E}-01$ & $1.38 \mathrm{E}+00$ & $4.33 \mathrm{E}-03$ & $1.25 \mathrm{E}+00$ & $1.23 \mathrm{E}+00$ \\
\hline 21 & $9.23 \mathrm{E}+00$ & $1.11 \mathrm{E}+00$ & $8.32 \mathrm{E}+00$ & $5.49 \mathrm{E}-01$ & $1.47 \mathrm{E}+00$ & $5.19 \mathrm{E}-03$ & $1.29 \mathrm{E}+00$ & $1.25 \mathrm{E}+00$ \\
\hline 22 & $8.97 \mathrm{E}+00$ & $1.12 \mathrm{E}+00$ & $7.69 \mathrm{E}+00$ & $5.55 \mathrm{E}-01$ & $1.55 \mathrm{E}+00$ & $6.08 \mathrm{E}-03$ & $1.33 \mathrm{E}+00$ & $1.27 \mathrm{E}+00$ \\
\hline 23 & $8.73 E+00$ & $1.14 \mathrm{E}+00$ & $7.18 \mathrm{E}+00$ & $5.62 \mathrm{E}-01$ & $1.63 \mathrm{E}+00$ & $6.98 \mathrm{E}-03$ & $1.37 \mathrm{E}+00$ & $1.29 \mathrm{E}+00$ \\
\hline 24 & $8.49 \mathrm{E}+00$ & $1.15 \mathrm{E}+00$ & $6.75 \mathrm{E}+00$ & $5.68 \mathrm{E}-01$ & $1.71 \mathrm{E}+00$ & $7.89 \mathrm{E}-03$ & $1.41 \mathrm{E}+00$ & $1.30 \mathrm{E}+00$ \\
\hline 25 & $8.25 \mathrm{E}+00$ & $1.17 \mathrm{E}+00$ & $6.40 \mathrm{E}+00$ & 5.75E-01 & $1.78 \mathrm{E}+00$ & 8.82E-03 & $1.45 \mathrm{E}+00$ & $1.32 \mathrm{E}+00$ \\
\hline 26 & $8.02 \mathrm{E}+00$ & $1.18 \mathrm{E}+00$ & $6.10 \mathrm{E}+00$ & $5.80 \mathrm{E}-01$ & $1.85 \mathrm{E}+00$ & $9.76 \mathrm{E}-03$ & $1.49 \mathrm{E}+00$ & $1.34 \mathrm{E}+00$ \\
\hline 27 & $7.78 \mathrm{E}+00$ & $1.19 \bar{E}+00$ & $5.83 \mathrm{E}+00$ & $5.86 \mathrm{E}-01$ & $1.91 \mathrm{E}+00$ & $1.07 \mathrm{E}-02$ & $1.53 \mathrm{E}+00$ & $1.35 \mathrm{E}+00$ \\
\hline 28 & $7.54 \mathrm{E}+00$ & $1.20 \mathrm{E}+00$ & $5.59 \mathrm{E}+00$ & $5.92 \mathrm{E}-01$ & $1.98 \mathrm{E}+00$ & $1.17 \mathrm{E}-02$ & $1.56 \mathrm{E}+00$ & $1.37 \mathrm{E}+00$ \\
\hline 29 & $7.32 \mathrm{E}+00$ & $1.22 \mathrm{E}+00$ & $5.37 \mathrm{E}+00$ & $5.97 \mathrm{E}-01$ & $2.04 \mathrm{E}+00$ & $1.27 \mathrm{E}-02$ & $1.60 \mathrm{E}+00$ & $1.38 \mathrm{E}+00$ \\
\hline 30 & $7.09 \mathrm{E}+00$ & $1.23 \mathrm{E}+00$ & $5.16 \mathrm{E}+00$ & $6.02 \mathrm{E}-01$ & $2.02 \mathrm{E}+00$ & $1.29 \mathrm{E}-02$ & $1.96 \mathrm{E}+00$ & $6.13 \mathrm{E}-01$ \\
\hline 31 & $6.88 \mathrm{E}+00$ & $1.24 \mathrm{E}+00$ & $4.97 \mathrm{E}+00$ & 6.07E-01 & $2.01 \mathrm{E}+00$ & $1.31 \mathrm{E}-02$ & $1.96 \mathrm{E}+00$ & $6.11 \mathrm{E}-01$ \\
\hline 32 & $6.67 \mathrm{E}+00$ & $1.25 \mathrm{E}+00$ & $4.78 \mathrm{E}+00$ & $6.12 \mathrm{E}-01$ & $2.00 \mathrm{E}+00$ & $1.33 \mathrm{E}-02$ & $1.95 \mathrm{E}+00$ & $6.09 \mathrm{E}-01$ \\
\hline 33 & $6.45 \mathrm{E}+00$ & $1.26 \mathrm{E}+00$ & $4.60 E+00$ & $6.17 \mathrm{E}-01$ & $1.98 \mathrm{E}+00$ & $1.35 \mathrm{E}-02$ & $1.94 \mathrm{E}+00$ & $6.07 \mathrm{E}-01$ \\
\hline 34 & $6.26 \mathrm{E}+00$ & $1.27 \mathrm{E}+00$ & $4.43 \mathrm{E}+00$ & $6.21 \mathrm{E}-01$ & $1.97 \mathrm{E}+00$ & $1.37 \mathrm{E}-02$ & $1.93 \mathrm{E}+00$ & $6.05 \mathrm{E}-01$ \\
\hline 35 & $6.07 \mathrm{E}+00$ & $1.28 \mathrm{E}+00$ & $4.27 \mathrm{E}+00$ & $6.26 \mathrm{E}-01$ & $1.95 \mathrm{E}+00$ & $1.39 \mathrm{E}-02$ & $1.93 E+00$ & $6.03 \mathrm{E}-01$ \\
\hline 36 & $5.90 E+00$ & $1.29 \mathrm{E}+00$ & $4.11 \mathrm{E}+00$ & $6.30 \mathrm{E}-01$ & $1.94 \mathrm{E}+00$ & $1.41 E-02$ & $1.92 \mathrm{E}+00$ & $6.01 \mathrm{E}-01$ \\
\hline 37 & $5.73 \mathrm{E}+00$ & $1.30 \mathrm{E}+00$ & $3.96 \mathrm{E}+00$ & $6.34 \mathrm{E}-01$ & $1.92 \mathrm{E}+00$ & $1.43 \mathrm{E}-02$ & $1.91 \mathrm{E}+00$ & $5.98 \mathrm{E}-01$ \\
\hline 38 & $5.58 \mathrm{E}+00$ & $1.31 \mathrm{E}+00$ & $3.81 \mathrm{E}+00$ & $6.38 \mathrm{E}-01$ & $1.91 \mathrm{E}+00$ & $1.45 \mathrm{E}-02$ & $1.91 \mathrm{E}+00$ & $5.96 \mathrm{E}-01$ \\
\hline
\end{tabular}




\begin{tabular}{|c|c|c|c|c|c|c|c|c|}
\hline 39 & $5.44 \mathrm{E}+00$ & $1.31 \mathrm{E}+00$ & $3.67 \mathrm{E}+00$ & $6.41 \mathrm{E}-01$ & $1.90 \mathrm{E}+00$ & $1.47 \mathrm{E}-02$ & $1.90 \mathrm{E}+00$ & $5.94 \mathrm{E}-0$ \\
\hline 40 & $5.31 \mathrm{E}+00$ & $1.32 \mathrm{E}+00$ & $3.54 \mathrm{E}+00$ & $6.45 \mathrm{E}-01$ & $1.88 \mathrm{E}+00$ & $1.49 \mathrm{E}-02$ & $1.89 \mathrm{E}+00$ & $5.92 \mathrm{E}-\mathrm{C}$ \\
\hline 41 & $5.18 \mathrm{E}+00$ & $1.33 \mathrm{E}+00$ & $3.42 \mathrm{E}+00$ & $6.48 \mathrm{E}-01$ & $1.87 \mathrm{E}+00$ & $1.51 \mathrm{E}-02$ & $1.89 \mathrm{E}+00$ & $5.90 \mathrm{E}-1$ \\
\hline 42 & $.06 \mathrm{E}+00$ & $34 \mathrm{E}+00$ & $3.30 \mathrm{E}+00$ & $6.52 \mathrm{E}-01$ & $1.91 \mathrm{E}+00$ & $1.61 \mathrm{E}-02$ & $1.85 \mathrm{E}+00$ & $.60 \mathrm{E}+$ \\
\hline 43 & $4.94 E+00$ & $1.34 \mathrm{E}+00$ & $3.19 \mathrm{E}+00$ & $6.55 \mathrm{E}-01$ & $1.94 \mathrm{E}+00$ & $1.72 \mathrm{E}-02$ & $1.86 \mathrm{E}+00$ & $1.61 \mathrm{E}+$ \\
\hline 44 & $4.83 E+00$ & $1.35 \mathrm{E}+00$ & $3.09 \mathrm{E}+00$ & $6.58 \mathrm{E}-01$ & $1.97 \mathrm{E}+00$ & $1.82 \mathrm{E}-02$ & $1.88 \mathrm{E}+00$ & $1.62 \mathrm{E}+0$ \\
\hline 45 & $4.72 \mathrm{E}+00$ & $1.36 \mathrm{E}+00$ & $2.99 \mathrm{E}+00$ & $6.61 \mathrm{E}-01$ & $2.01 \mathrm{E}+00$ & $1.93 \mathrm{E}-02$ & $1.90 \mathrm{E}+00$ & $1.62 \mathrm{E}+\mathrm{c}$ \\
\hline 46 & $4.62 \mathrm{E}+00$ & $1.37 \mathrm{E}+00$ & $2.90 \mathrm{E}+00$ & $6.64 \mathrm{E}-01$ & $2.04 \mathrm{E}+00$ & $2.03 \mathrm{E}-02$ & $1.91 \mathrm{E}+00$ & $1.63 \mathrm{E}+\mathrm{C}$ \\
\hline 47 & $4.53 \mathrm{E}+00$ & $1.37 \mathrm{E}+00$ & $2.81 \mathrm{E}+00$ & $6.67 \mathrm{E}-01$ & $2.07 \mathrm{E}+00$ & $2.14 \mathrm{E}-02$ & $1.93 \mathrm{E}+00$ & $1.64 \mathrm{E}+\mathrm{C}$ \\
\hline 48 & $4.43 \mathrm{E}+00$ & $1.38 \mathrm{E}+00$ & $2.73 \mathrm{E}+00$ & $6.70 \mathrm{E}-01$ & $2.09 \mathrm{E}+00$ & $2.25 \mathrm{E}-02$ & $1.94 \mathrm{E}+00$ & $1.64 \mathrm{E}+$ \\
\hline 49 & $4.34 \mathrm{E}+00$ & $1.38 \mathrm{E}+00$ & $2.65 \mathrm{E}+00$ & $6.73 \mathrm{E}-01$ & $2.12 \mathrm{E}+00$ & $2.35 \mathrm{E}-02$ & $1.96 \mathrm{E}+00$ & $1.65 \mathrm{E}+0$ \\
\hline 50 & $4.27 \mathrm{E}+00$ & $1.39 \mathrm{E}+00$ & $2.57 \mathrm{E}+00$ & $6.75 \mathrm{E}-01$ & $2.15 \mathrm{E}+00$ & $2.46 \mathrm{E}-02$ & $1.97 \mathrm{E}+00$ & $1.66 \mathrm{E}+0$ \\
\hline 51 & $4.20 \mathrm{E}+00$ & $1.40 \mathrm{E}+00$ & $2.50 \mathrm{E}+00$ & $6.78 E-01$ & $2.17 \mathrm{E}+00$ & $2.57 \mathrm{E}-02$ & $1.98 \mathrm{E}+00$ & $1.66 \mathrm{E}+$ \\
\hline 52 & $4.14 \mathrm{E}+00$ & $1.40 \mathrm{E}+00$ & $2.43 \mathrm{E}+00$ & $6.80 \mathrm{E}-01$ & $2.20 \mathrm{E}+00$ & $2.68 \mathrm{E}-02$ & $2.00 \mathrm{E}+00$ & $1.67 \mathrm{E}+0$ \\
\hline 53 & $4.08 \mathrm{E}+00$ & $1.41 \mathrm{E}+00$ & $2.37 \mathrm{E}+00$ & $6.83 \mathrm{E}-01$ & $2.22 \mathrm{E}+00$ & $2.79 \mathrm{E}-02$ & $2.01 \mathrm{E}+00$ & $1.67 \mathrm{E}+\mathrm{c}$ \\
\hline 54 & $4.03 \mathrm{E}+00$ & $1.41 \mathrm{E}+00$ & $2.31 \mathrm{E}+00$ & $6.85 \mathrm{E}-01$ & $2.20 \mathrm{E}+00$ & $2.81 \mathrm{E}-02$ & $2.13 \mathrm{E}+00$ & $6.70 \mathrm{E}-\mathrm{C}$ \\
\hline 55 & $3.98 \mathrm{E}+00$ & $1.42 \mathrm{E}+00$ & $2.26 \mathrm{E}+00$ & $6.88 \mathrm{E}-01$ & $2.18 \mathrm{E}+00$ & $2.83 \mathrm{E}-02$ & $2.13 \mathrm{E}+00$ & $6.67 \mathrm{E}-0$ \\
\hline 56 & $3.93 \mathrm{E}+00$ & $1.42 \mathrm{E}+00$ & $2.21 \mathrm{E}+00$ & $6.90 \mathrm{E}-01$ & $2.17 \mathrm{E}+00$ & $2.85 \mathrm{E}-02$ & $2.12 \mathrm{E}+00$ & $6.65 \mathrm{E}-0$ \\
\hline 57 & $3.89 \mathrm{E}+00$ & $1.43 \mathrm{E}+00$ & $2.17 \mathrm{E}+00$ & 6.92E-01 & $2.15 E+00$ & $2.87 \mathrm{E}-02$ & $2.11 \mathrm{E}+00$ & $6.62 \mathrm{E}-\mathrm{C}$ \\
\hline 58 & $3.84 \mathrm{E}+00$ & $1.43 \mathrm{E}+00$ & $2.13 \mathrm{E}+00$ & $6.95 \mathrm{E}-01$ & $2.13 E+00$ & $2.89 \mathrm{E}-02$ & $2.10 \mathrm{E}+00$ & $6.60 \mathrm{E}-0$ \\
\hline 59 & $3.80 \mathrm{E}+00$ & $1.44 \mathrm{E}+00$ & $2.09 \mathrm{E}+00$ & $6.97 \mathrm{E}-01$ & $2.12 \mathrm{E}+00$ & 2.92E-02 & $2.09 \mathrm{E}+00$ & $6.57 \mathrm{E}-0$ \\
\hline 60 & $3.76 \mathrm{E}+00$ & $1.44 \mathrm{E}+00$ & $2.06 \mathrm{E}+00$ & $6.99 \mathrm{E}-01$ & $2.10 \mathrm{E}+00$ & $2.94 \mathrm{E}-02$ & $2.08 \mathrm{E}+00$ & $6.54 \mathrm{E}-0$ \\
\hline 61 & $3.72 \mathrm{E}+00$ & $1.45 \mathrm{E}+00$ & $2.02 \mathrm{E}+00$ & $7.01 \mathrm{E}-01$ & $2.08 \mathrm{E}+00$ & $2.96 \mathrm{E}-02$ & $2.08 \mathrm{E}+00$ & $6.52 \mathrm{E}-0$ \\
\hline 62 & $3.68 \mathrm{E}+00$ & $1.45 \mathrm{E}+00$ & $1.99 \mathrm{E}+00$ & $7.03 E-01$ & $2.07 \mathrm{E}+00$ & $2.98 \mathrm{E}-02$ & $2.07 \mathrm{E}+00$ & $\overline{6.49 \mathrm{E}-0}$ \\
\hline 63 & $3.64 \mathrm{E}+00$ & $1.46 \mathrm{E}+00$ & $1.96 \mathrm{E}+00$ & $7.05 \mathrm{E}-01$ & $2.05 \mathrm{E}+00$ & $3.00 \mathrm{E}-02$ & $2.06 \mathrm{E}+00$ & 6.47 \\
\hline 64 & $3.61 \mathrm{E}+00$ & $1.46 \mathrm{E}+00$ & $1.93 \mathrm{E}+00$ & $7.08 \mathrm{E}-01$ & $2.03 E+00$ & $3.02 \mathrm{E}-02$ & $2.05 \mathrm{E}+00$ & $6.44 \mathrm{E}-0$ \\
\hline 65 & $3.57 \mathrm{E}+00$ & $1.47 \mathrm{E}+00$ & $1.90 \mathrm{E}+00$ & $7.10 \mathrm{E}-01$ & $2.02 \mathrm{E}+00$ & $3.04 \mathrm{E}-02$ & $2.04 \mathrm{E}+00$ & $6.42 \mathrm{E}-0$ \\
\hline 66 & $3.54 \mathrm{E}+00$ & $1.47 \mathrm{E}+00$ & $1.88 \mathrm{E}+00$ & 7.12 & $2.04 \mathrm{E}+00$ & $3.15 \mathrm{E}-02$ & $1.97 \mathrm{E}+00$ & $1.72 \mathrm{E}+0$ \\
\hline 67 & $3.50 \mathrm{E}+00$ & $1.48 \mathrm{E}+00$ & $1.85 \mathrm{E}+00$ & $7.14 \mathrm{E}-01$ & $2.05 \mathrm{E}+00$ & $3.26 \mathrm{E}-02$ & $1.98 \mathrm{E}+00$ & $1.73 E+0$ \\
\hline 68 & $3.47 \mathrm{E}+00$ & $1.48 \mathrm{E}+00$ & $1.83 \mathrm{E}+00$ & $7.16 \mathrm{E}-01$ & $2.07 \mathrm{E}+00$ & $3.37 \mathrm{E}-02$ & $1.99 \mathrm{E}+00$ & $1.73 \mathrm{E}+0$ \\
\hline 69 & $3.44 \mathrm{E}+00$ & $1.48 \mathrm{E}+00$ & $1.80 \mathrm{E}+00$ & $7.17 \mathrm{E}-01$ & $2.09 \mathrm{E}+00$ & $3.48 \mathrm{E}-02$ & $2.00 \mathrm{E}+00$ & $1.73 E+0$ \\
\hline 168 & $7.23 \mathrm{E}-01$ & $1.54 \mathrm{E}+00$ & $7.85 \mathrm{E}-01$ & $8.35 \mathrm{E}-01$ & $1.61 \mathrm{E}+00$ & $1.37 \mathrm{E}-01$ & $1.76 \mathrm{E}+00$ & $1.69 \mathrm{E}+0$ \\
\hline 336 & $3.93 \mathrm{E}-01$ & $1.30 \mathrm{E}+00$ & $4.23 \mathrm{E}-01$ & $8.96 \mathrm{E}-01$ & $8.43 \mathrm{E}-01$ & $2.65 \mathrm{E}-01$ & $1.15 \mathrm{E}+00$ & $1.35 \mathrm{E}+0$ \\
\hline 504 & $2.92 \mathrm{E}-01$ & $1.09 \mathrm{E}+00$ & $2.93 \mathrm{E}-01$ & 9.34E-01 & $4.79 \mathrm{E}-01$ & $3.40 \mathrm{E}-01$ & $6.20 \mathrm{E}-01$ & $9.80 \mathrm{E}-0$ \\
\hline 720 & $2.13 E-01$ & 8.55E-01 & $1.97 \mathrm{E}-01$ & $9.67 \mathrm{E}-01$ & $2.65 \mathrm{E}-01$ & $3.84 E-01$ & $3.48 \mathrm{E}-01$ & $7.03 E-0$ \\
\hline 2160 & $3.99 \mathrm{E}-02$ & $1.70 \mathrm{E}-01$ & $2.22 \mathrm{E}-02$ & $1.03 \mathrm{E}+00$ & $2.41 \mathrm{E}-02$ & $2.33 \mathrm{E}-01$ & $8.49 \mathrm{E}-02$ & $2.44 \mathrm{E}-0$ \\
\hline 4320 & $3.84 \mathrm{E}-03$ & $1.53 \mathrm{E}-02$ & $1.12 \mathrm{E}-01$ & $9.77 \mathrm{E}-01$ & $3.97 \mathrm{E}-03$ & $5.60 \mathrm{E}-02$ & $1.02 \mathrm{E}-02$ & $3.76 \mathrm{E}-0$ \\
\hline 4800 & $2.28 \mathrm{E}-03$ & $8.98 \mathrm{E}-03$ & $1.11 \mathrm{E}-01$ & $9.74 \mathrm{E}-01$ & $2.88 \mathrm{E}-03$ & $4.03 \mathrm{E}-02$ & $3.39 \mathrm{E}-03$ & $1.22 \mathrm{E}-02$ \\
\hline 8760 & $3.14 \mathrm{E}-05$ & $1.17 \mathrm{E}-04$ & $1.08 \mathrm{E}-01$ & $9.50 \mathrm{E}-01$ & $2.36 \mathrm{E}-04$ & $2.69 \mathrm{E}-03$ & $1.06 \mathrm{E}-03$ & $3.60 \mathrm{E}-0$ \\
\hline $\mathbf{B T}$ & $\begin{array}{l}E 6 \\
\text { L }\end{array}$ & $\begin{array}{l}8.88 \mathrm{E}+4 \\
\mathrm{Bg} / \mathrm{kg}\end{array}$ & $8 \mathrm{~Bq} / \mathrm{g}$ & & & & & \\
\hline & $\begin{array}{l}5.9 \mathrm{E} 6 \\
\mathrm{pCi} / \mathrm{L}\end{array}$ & $\begin{array}{l}2.18 \mathrm{E}+5 \\
\mathrm{~Bq} / \mathrm{kg}\end{array}$ & $3 \mathrm{~Bq} / \mathrm{g}$ & & & & & \\
\hline & \multicolumn{2}{|c|}{ VEGETABLES } & \multicolumn{2}{|c|}{ WHEAT SEED } & \multicolumn{2}{|l|}{ MEAT } & \multicolumn{2}{|l|}{ MILK } \\
\hline \multirow[t]{2}{*}{ HOUR } & $\overline{0}$ & OBT & 0 & $\overline{\mathrm{OBT}}$ & TO & I & TO & $\overline{B T}$ \\
\hline & $(\mathrm{Bq} / \mathrm{g})$ & $(\mathrm{Bq} / \mathrm{g})$ & $(\mathrm{Bq} / \mathrm{g})$ & $(\mathrm{Bq} / \mathrm{g})$ & $q / g)$ & $q / g)$ & (q/g) & $\mathrm{Bq} / \mathrm{g})$ \\
\hline annic & & & & & & & & \\
\hline
\end{tabular}




\begin{tabular}{|c|c|c|c|c|c|c|c|c|}
\hline $6 / 8 / 9 / 9$ & $7.21 \mathrm{E}+01$ & $5.98 \mathrm{E}-01$ & $5.57 \mathrm{E}+01$ & $3.43 \mathrm{E}-01$ & $1.13 \mathrm{E}+00$ & $8.87 \mathrm{E}-04$ & $1.09 \mathrm{E}+00$ & $3.39 \mathrm{E}-01$ \\
\hline SUM & $7.27 \mathrm{E}+01$ & & $5.60 \mathrm{E}+01$ & & $1.13 \mathrm{E}+00$ & & $1.43 \mathrm{E}+00$ & \\
\hline $\mathrm{Ci} / \mathrm{g}$ & $1.96 \mathrm{E}-09$ & & $1.51 \mathrm{E}-09$ & & $3.05 \mathrm{E}-11$ & & $3.85 \mathrm{E}-11$ & \\
\hline & & & & & & & & \\
\hline DIL HTO & $5.9 \mathrm{E}-09$ & & & & & & & \\
\hline \multicolumn{9}{|l|}{$(\mathrm{Ci} / \mathrm{g})$} \\
\hline & & & & & & & & \\
\hline 168 & $7.23 \mathrm{E}-01$ & $1.54 \mathrm{E}+00$ & $7.85 \mathrm{E}-01$ & $8.35 \mathrm{E}-01$ & $1.61 \mathrm{E}+00$ & $1.37 \mathrm{E}-01$ & $1.76 \mathrm{E}+00$ & $1.69 \mathrm{E}+00$ \\
\hline SUM & $2.26 \mathrm{E}+00$ & & $1.62 \mathrm{E}+00$ & & $1.75 \mathrm{E}+00$ & & $3.45 \mathrm{E}+00$ & \\
\hline $\mathrm{Ci} / \mathrm{g}$ & $6.12 \mathrm{E}-11$ & & $4.38 \mathrm{E}-11$ & & $4.73 \mathrm{E}-11$ & & $9.32 \mathrm{E}-11$ & \\
\hline DIL OBT & $24 F_{-00}$ & & & & & & & \\
\hline \begin{tabular}{|l}
$\mathrm{Ci} / \mathrm{g}$ \\
\end{tabular} & & & & & & & & \\
\hline Civg & & $\dot{-}$ & & & & & & \\
\hline 8760 & $3.14 \mathrm{E}-05$ & $1.17 \mathrm{E}-04$ & $1.08 \mathrm{E}-01$ & $9.50 \mathrm{E}-01$ & $2.36 \mathrm{E}-04$ & $2.69 \mathrm{E}-03$ & $1.06 \mathrm{E}-03$ & $3.60 \mathrm{E}-03$ \\
\hline SUM & $1.48 \mathrm{E}-04$ & & $1.06 \mathrm{E}+00$ & & $2.92 \mathrm{E}-03$ & & $4.66 \mathrm{E}-03$ & \\
\hline $\mathrm{Ci} / \mathrm{g}$ & $4.00 \mathrm{E}-15$ & & $2.86 \mathrm{E}-11$ & & $7.90 \mathrm{E}-14$ & & $1.26 \mathrm{E}-13$ & \\
\hline DIL OBT & C $A \mathrm{~F}_{00}$ & & & & & & & \\
\hline \begin{tabular}{|ll} 
\\
\end{tabular} & $2.4 E-09$ & & & & & & & \\
\hline $\mathrm{Ci} / \mathrm{g}$ & & & & & & & & \\
\hline
\end{tabular}




\begin{tabular}{|c|c|c|c|c|c|c|c|c|}
\hline & \multicolumn{6}{|c|}{ SRTC Release Scenario 1-RD-3, Average Met Conditions } & & \\
\hline & \multicolumn{4}{|c|}{ Concentration Values for $100 \mathrm{~km}$ from release } & & & & \\
\hline \multicolumn{9}{|c|}{ Only Wheat Seed is examined since this is the edible portion of the Wheat. The "WHEAT" category is } \\
\hline \multicolumn{9}{|c|}{ actually the unedible plant body and is not considered in the dose estimate. } \\
\hline & & & & & & & & \\
\hline & \multicolumn{2}{|c|}{ VEGETABLES } & \multicolumn{2}{|c|}{ WHEAT SEED } & \multicolumn{2}{|l|}{ MEAT } & \multicolumn{2}{|l|}{ MILK } \\
\hline \multirow[t]{2}{*}{ HOUR } & HTO & $\widehat{\text { OBT }}$ & HTO & OBT & HTO & OBT & HTO & OBT \\
\hline & $(\mathrm{Bq} / \mathrm{g})$ & $(\mathrm{Bq} / \mathrm{g})$ & $(\mathrm{Bq} / \mathrm{g})$ & $(\mathrm{Bq} / \mathrm{g})$ & $(\mathrm{Bq} / \mathrm{g})$ & $(\overline{B q} / \mathrm{g}$ & $(\mathrm{Bq} / \mathrm{g})$ & $(\mathrm{Bq} / \mathrm{g}$ \\
\hline & & & & & & & & \\
\hline 1 & $2.37 \mathrm{E}+00$ & $0.00 \mathrm{E}+00$ & $0.00 \mathrm{E}+00$ & $0.00 \mathrm{E}+00$ & $2.24 \mathrm{E}-03$ & $2.41 \mathrm{E}-07$ & $2.71 E-04$ & 8.43E-0 \\
\hline 2 & $4.21 \mathrm{E}+00$ & $4.24 \mathrm{E}-03$ & $2.73 \mathrm{E}-01$ & $1.04 \mathrm{E}-03$ & $2.38 \mathrm{E}-02$ & $4.97 \mathrm{E}-06$ & $2.81 E-03$ & $\overline{E-03}$ \\
\hline 3 & $4.30 \mathrm{E}+00$ & $1.18 \mathrm{E}-02$ & $7.82 \mathrm{E}-01$ & $3.28 \mathrm{E}-03$ & $6.19 \mathrm{E}-02$ & -05 & 8.53E-03 & 3 \\
\hline 4 & $5.81 \mathrm{E}+00$ & $1.95 \mathrm{E}-02$ & $1.30 \mathrm{E}+00$ & $6.12 \mathrm{E}-03$ & $1.05 \mathrm{E}-01$ & $4.04 \mathrm{E}-05$ & $1.70 \mathrm{E}-02$ & $6 E-03$ \\
\hline 5 & $5.51 \mathrm{E}+00$ & $2.98 \mathrm{E}-02$ & $1.93 \mathrm{E}+00$ & $9.98 \mathrm{E}-03$ & $1.61 \mathrm{E}-01$ & $7.49 \mathrm{E}-05$ & $2.86 E-02$ & $1.26 \mathrm{E}-02$ \\
\hline 6 & $7.88 \mathrm{E}+00$ & $3.96 \mathrm{E}-02$ & $2.47 \mathrm{E}+00$ & $1.42 \mathrm{E}-02$ & $1.65 \mathrm{E}-01$ & $9.26 \mathrm{E}-05$ & $1.60 \mathrm{E}-01$ & $4.99 \mathrm{E}-0$ \\
\hline 7 & $7.34 \mathrm{E}+00$ & $5.37 \mathrm{E}-02$ & $3.19 \mathrm{E}+00$ & $1.97 \mathrm{E}-02$ & $1.69 \mathrm{E}-01$ & $1.11 \mathrm{E}-04$ & $1.62 \mathrm{E}-01$ & $\overline{\mathrm{E}-02}$ \\
\hline 8 & $7.58 \mathrm{E}+00$ & $6.68 \mathrm{E}-02$ & $3.77 \mathrm{E}+00$ & $2.54 \mathrm{E}-02$ & $1.73 \mathrm{E}-01$ & $1.29 \mathrm{E}-04$ & $1.64 \mathrm{E}-01$ & $\mathrm{E}-02$ \\
\hline 9 & $1.03 \mathrm{E}+01$ & $8.03 \mathrm{E}-02$ & $4.27 \mathrm{E}+00$ & $3.15 \mathrm{E}-02$ & $1.79 \mathrm{E}-01$ & & $1.66 \mathrm{E}-01$ & $c=0$ \\
\hline 10 & $9.49 E+00$ & $1.02 \mathrm{E}-01$ & $5.25 \mathrm{E}+00$ & $4.00 \mathrm{E}-02$ & $1.86 \mathrm{E}-01$ & -04 & $1.69 \mathrm{E}-01$ & $\overline{\mathrm{E}-0}$ \\
\hline 11 & $2.04 \mathrm{E}+01$ & $2.06 \mathrm{E}-01$ & $1.21 \mathrm{E}+01$ & $7.19 \mathrm{E}-02$ & $2.36 \mathrm{E}-01$ & $1.94 \mathrm{E}-04$ & $1.79 \mathrm{E}-01$ & $5.58 \mathrm{E}-02$ \\
\hline 12 & $2.71 \mathrm{E}+01$ & $3.57 \mathrm{E}-01$ & $2.27 \mathrm{E}+01$ & $1.26 \mathrm{E}-01$ & $3.00 \mathrm{E}-01$ & -04 & $1.95 \mathrm{E}-01$ & $\mathrm{E}-\mathrm{U}$ \\
\hline 13 & $1.26 \mathrm{E}+01$ & $4.05 \mathrm{E}-01$ & $2.57 \mathrm{E}+01$ & $1.62 \mathrm{E}-01$ & $3.02 \mathrm{E}-01$ & $2.58 \mathrm{E}-04$ & $2.07 \mathrm{E}-01$ & $\overline{E-0}$ \\
\hline 14 & $8.63 \mathrm{E}+00$ & $4.27 \mathrm{E}-01$ & $2.48 \mathrm{E}+01$ & $1.87 \mathrm{E}-01$ & $3.04 \mathrm{E}-01$ & $2.91 \mathrm{E}-04$ & $2.17 \mathrm{E}-01$ & $6.76 \mathrm{E}-02$ \\
\hline 15 & $7.41 \mathrm{E}+00$ & $4.42 \mathrm{E}-01$ & $2.22 \mathrm{E}+01$ & $2.05 \mathrm{E}-01$ & $3.05 \mathrm{E}-01$ & $3.23 \mathrm{E}-04$ & $2.25 \mathrm{E}-01$ & $7.00 \mathrm{E}-02$ \\
\hline 16 & $6.93 \mathrm{E}+00$ & $4.55 \mathrm{E}-01$ & $1.92 \mathrm{E}+01$ & $2.18 \mathrm{E}-01$ & $3.06 \mathrm{E}-01$ & $3.56 \mathrm{E}-04$ & $2.31 \mathrm{E}-01$ & $7.20 \mathrm{E}-0$ \\
\hline 17 & $6.68 \mathrm{E}+00$ & $4.66 \mathrm{E}-01$ & $1.62 \mathrm{E}+01$ & $2.29 \mathrm{E}-01$ & $3.07 \mathrm{E}-01$ & $3.89 \mathrm{E}-04$ & $2.37 \mathrm{E}-01$ & $7.38 \mathrm{E}-02$ \\
\hline 18 & $6.51 \mathrm{E}+00$ & $4.78 \mathrm{E}-01$ & $1.36 \mathrm{E}+01$ & $2.37 \mathrm{E}-01$ & $3.77 \mathrm{E}-01$ & $7.36 \mathrm{E}-04$ & $3.65 \mathrm{E}-01$ & $4.86 \mathrm{E}-0$ \\
\hline 19 & $6.37 \mathrm{E}+00$ & $4.89 \mathrm{E}-01$ & $1.15 \mathrm{E}+01$ & $2.44 \mathrm{E}-01$ & $4.43 \mathrm{E}-01$ & $1.10 \mathrm{E}-03$ & $3.97 \mathrm{E}-01$ & $5.00 \mathrm{E}-01$ \\
\hline 20 & $6.25 \mathrm{E}+00$ & $4.99 \mathrm{E}-01$ & $9.73 \mathrm{E}+00$ & $2.50 \mathrm{E}-01$ & $5.06 \mathrm{E}-01$ & $\mathrm{E}-03$ & $4.28 \mathrm{E}-01$ & $5.14 \mathrm{E}-01$ \\
\hline 21 & $6.14 \mathrm{E}+00$ & $5.10 \mathrm{E}-01$ & $8.35 \mathrm{E}+00$ & $2.55 \mathrm{E}-01$ & $5.67 \mathrm{E}-01$ & $1.86 \mathrm{E}-03$ & $4.59 \mathrm{E}-01$ & $5.27 \mathrm{E}-01$ \\
\hline 22 & $6.02 \mathrm{E}+00$ & $5.20 \mathrm{E}-01$ & $7.27 \mathrm{E}+00$ & $2.61 \mathrm{E}-01$ & $6.25 \mathrm{E}-01$ & $2.26 \mathrm{E}-03$ & $4.88 \mathrm{E}-01$ & $5.40 \mathrm{E}-01$ \\
\hline 23 & $5.91 \mathrm{E}+00$ & $5.30 \mathrm{E}-01$ & $6.44 \mathrm{E}+00$ & $2.66 \mathrm{E}-01$ & $6.82 \mathrm{E}-01$ & $2.67 \mathrm{E}-03$ & $5.17 \mathrm{E}-01$ & $5.53 \mathrm{E}-01$ \\
\hline 24 & $5.80 \mathrm{E}+00$ & $5.40 \mathrm{E}-01$ & $5.80 \mathrm{E}+00$ & $2.70 \mathrm{E}-01$ & $7.38 \mathrm{E}-01$ & $3.09 \mathrm{E}-03$ & $5.45 \mathrm{E}-01$ & $5.65 \mathrm{E}-01$ \\
\hline 25 & $5.69 \mathrm{E}+00$ & $5.50 \mathrm{E}-01$ & $5.30 \mathrm{E}+00$ & $2.75 \mathrm{E}-01$ & $7.91 \mathrm{E}-01$ & $3.52 \mathrm{E}-03$ & $5.73 \mathrm{E}-01$ & 5.7 \\
\hline 26 & $5.58 \mathrm{E}+00$ & $5.59 \mathrm{E}-01$ & $4.91 E+00$ & $2.79 \mathrm{E}-01$ & $8.43 \mathrm{E}-01$ & $3.96 \mathrm{E}-03$ & $6.00 \mathrm{E}-01$ & $5.90 \mathrm{E}-01$ \\
\hline 27 & $5.46 \mathrm{E}+00$ & $5.69 \mathrm{E}-01$ & $4.61 \mathrm{E}+00$ & $2.84 \mathrm{E}-01$ & $8.93 \mathrm{E}-01$ & $4.42 \mathrm{E}-03$ & $6.27 \mathrm{E}-01$ & $6.01 \mathrm{E}-0$ \\
\hline 28 & $5.33 \mathrm{E}+00$ & $5.78 \mathrm{E}-01$ & $4.36 \mathrm{E}+00$ & $2.88 \mathrm{E}-01$ & $9.42 \mathrm{E}-01$ & $4.88 \mathrm{E}-03$ & $6.53 \mathrm{E}-01$ & $6.13 \mathrm{E}-0$ \\
\hline 29 & $5.21 \mathrm{E}+00$ & $5.86 \mathrm{E}-01$ & $4.16 \mathrm{E}+00$ & $2.92 \mathrm{E}-01$ & $9.88 \mathrm{E}-01$ & $5.35 \mathrm{E}-03$ & $6.78 \mathrm{E}-01$ & $6.24 \mathrm{E}-0$ \\
\hline 30 & $5.09 \mathrm{E}+00$ & $5.95 \mathrm{E}-01$ & $3.98 \mathrm{E}+00$ & $2.96 \mathrm{E}-01$ & $9.82 \mathrm{E}-01$ & $5.46 \mathrm{E}-03$ & $9.52 \mathrm{E}-01$ & $2.98 \mathrm{E}-01$ \\
\hline 31 & $4.96 \mathrm{E}+00$ & $6.03 \mathrm{E}-01$ & $3.83 \mathrm{E}+00$ & $3.00 \mathrm{E}-01$ & $9.77 \mathrm{E}-01$ & $5.56 \mathrm{E}-03$ & $9.49 \mathrm{E}-01$ & $2.97 \mathrm{E}-01$ \\
\hline 32 & $4.83 \mathrm{E}+00$ & $6.11 \mathrm{E}-01$ & $3.70 \mathrm{E}+00$ & $3.03 \mathrm{E}-01$ & $9.71 \mathrm{E}-01$ & $5.66 \mathrm{E}-03$ & $9.47 \mathrm{E}-01$ & $2.96 \mathrm{E}-01$ \\
\hline 33 & $4.70 \mathrm{E}+00$ & $6.19 \mathrm{E}-01$ & $3.58 \mathrm{E}+00$ & $3.07 \mathrm{E}-01$ & $9.65 \mathrm{E}-01$ & $5.76 \mathrm{E}-03$ & $9.44 \mathrm{E}-01$ & $2.95 \mathrm{E}-01$ \\
\hline 34 & $4.58 \mathrm{E}+00$ & $6.27 \mathrm{E}-01$ & $3.46 \mathrm{E}+00$ & $3.11 \mathrm{E}-01$ & $9.59 \mathrm{E}-01$ & $5.86 \mathrm{E}-03$ & $9.41 \mathrm{E}-01$ & $2.94 \mathrm{E}-01$ \\
\hline 35 & $4.45 \mathrm{E}+00$ & $6.34 \mathrm{E}-01$ & $3.35 \mathrm{E}+00$ & $3.14 \mathrm{E}-01$ & $9.53 \mathrm{E}-01$ & $5.95 \mathrm{E}-03$ & $9.38 \mathrm{E}-01$ & $2.93 \mathrm{E}-01$ \\
\hline 36 & $4.33 \mathrm{E}+00$ & $6.41 \mathrm{E}-01$ & $3.25 \mathrm{E}+00$ & $3.17 \mathrm{E}-01$ & $9.47 \mathrm{E}-01$ & $6.05 \mathrm{E}-03$ & $9.35 \mathrm{E}-01$ & $2.92 \mathrm{E}-01$ \\
\hline 37 & $4.21 \mathrm{E}+00$ & $6.48 \mathrm{E}-01$ & $3.15 \mathrm{E}+00$ & $3.21 \mathrm{E}-01$ & $9.41 \mathrm{E}-01$ & $6.15 \mathrm{E}-03$ & $9.32 \mathrm{E}-01$ & $2.91 \mathrm{E}-01$ \\
\hline 38 & $4.10 \mathrm{E}+00$ & $6.55 \mathrm{E}-01$ & $3.05 \mathrm{E}+00$ & $3.24 \mathrm{E}-01$ & $9.35 \mathrm{E}-01$ & $6.25 \mathrm{E}-03$ & $9.29 \mathrm{E}-01$ & $2.91 \mathrm{E}-0$ \\
\hline
\end{tabular}




\begin{tabular}{|c|c|c|c|c|c|c|c|c|}
\hline 39 & $4.00 \mathrm{E}+00$ & $6.61 \mathrm{E}-01$ & $2.95 \mathrm{E}+00$ & $3.27 \mathrm{E}-01$ & $9.29 \mathrm{E}-01$ & $6.34 \mathrm{E}-03$ & $9.26 \mathrm{E}-01$ & $2.90 \mathrm{E}-01$ \\
\hline 40 & $3.90 \mathrm{E}+00$ & $6.68 \mathrm{E}-01$ & $2.86 \mathrm{E}+00$ & $3.30 \mathrm{E}-01$ & $9.23 \mathrm{E}-01$ & $6.44 \mathrm{E}-03$ & $9.24 \mathrm{E}-01$ & $2.89 \mathrm{E}-01$ \\
\hline 41 & $3.81 \mathrm{E}+00$ & $6.74 \mathrm{E}-01$ & $2.78 \mathrm{E}+00$ & $3.33 \mathrm{E}-01$ & $9.17 \mathrm{E}-01$ & $6.53 \mathrm{E}-03$ & $9.21 \mathrm{E}-01$ & $2.88 \mathrm{E}-01$ \\
\hline 42 & $3.71 \mathrm{E}+00$ & $6.80 \mathrm{E}-01$ & $2.69 \mathrm{E}+00$ & $3.35 \mathrm{E}-01$ & $9.48 \mathrm{E}-01$ & $7.06 \mathrm{E}-03$ & $9.19 \mathrm{E}-01$ & $8.08 \mathrm{E}-01$ \\
\hline 43 & $3.62 E+00$ & $6.85 \mathrm{E}-01$ & $2.61 \mathrm{E}+00$ & $3.38 \mathrm{E}-01$ & $9.78 \mathrm{E}-01$ & $7.59 \mathrm{E}-03$ & $9.33 \mathrm{E}-01$ & $8.14 \mathrm{E}-01$ \\
\hline 44 & $3.54 \mathrm{E}+00$ & $6.91 \mathrm{E}-01$ & $2.53 \mathrm{E}+00$ & $3.41 \mathrm{E}-01$ & $1.01 \mathrm{E}+00$ & $8.13 \mathrm{E}-03$ & $9.47 \mathrm{E}-01$ & $8.20 \mathrm{E}-01$ \\
\hline 45 & $3.46 \mathrm{E}+00$ & $6.96 \mathrm{E}-01$ & $2.46 \mathrm{E}+00$ & $3.43 \mathrm{E}-01$ & $1.03 \mathrm{E}+00$ & $8.67 E-03$ & $9.61 \mathrm{E}-01$ & $8.26 \mathrm{E}-01$ \\
\hline 46 & $3.38 \mathrm{E}+00$ & $7.02 \mathrm{E}-01$ & $2.39 \mathrm{E}+00$ & $3.46 \mathrm{E}-01$ & $1.06 \mathrm{E}+00$ & $9.22 \mathrm{E}-03$ & $9.74 \mathrm{E}-01$ & $8.32 \mathrm{E}-01$ \\
\hline 47 & $3.30 \mathrm{E}+00$ & $7.07 \mathrm{E}-01$ & $2.32 \mathrm{E}+00$ & $3.48 \mathrm{E}-01$ & $1.09 \mathrm{E}+00$ & $9.77 \mathrm{E}-03$ & $9.87 \mathrm{E}-01$ & $8.37 \mathrm{E}-01$ \\
\hline 48 & $3.23 \mathrm{E}+00$ & $7.12 \mathrm{E}-01$ & $2.25 \mathrm{E}+00$ & $3.50 \mathrm{E}-01$ & $1.11 \mathrm{E}+00$ & $1.03 \mathrm{E}-02$ & $9.99 \mathrm{E}-01$ & $8.43 \mathrm{E}-01$ \\
\hline 49 & $3.16 \mathrm{E}+00$ & $7.16 \mathrm{E}-01$ & $2.19 \mathrm{E}+00$ & $3.53 \mathrm{E}-01$ & $1.13 \mathrm{E}+00$ & $1.09 \mathrm{E}-02$ & $1.01 \mathrm{E}+00$ & $8.48 \mathrm{E}-01$ \\
\hline 50 & $3.09 \mathrm{E}+00$ & $7.21 \mathrm{E}-01$ & $2.13 \mathrm{E}+00$ & $3.55 \mathrm{E}-01$ & $1.15 \mathrm{E}+00$ & $1.15 \mathrm{E}-02$ & $1.02 \mathrm{E}+00$ & $8.53 \mathrm{E}-01$ \\
\hline 51 & $3.04 \mathrm{E}+00$ & $7.26 \mathrm{E}-01$ & $2.07 \mathrm{E}+00$ & $3.57 \mathrm{E}-01$ & $1.18 \mathrm{E}+00$ & $1.20 \mathrm{E}-02$ & $1.04 \mathrm{E}+00$ & $8.58 \mathrm{E}-01$ \\
\hline 52 & $2.99 \mathrm{E}+00$ & $7.30 \mathrm{E}-01$ & $2.01 \mathrm{E}+00$ & $3.59 \mathrm{E}-01$ & $1.20 \mathrm{E}+00$ & $1.26 \mathrm{E}-02$ & $1.05 \mathrm{E}+00$ & $8.63 \mathrm{E}-01$ \\
\hline 53 & $2.94 \mathrm{E}+00$ & $7.35 \mathrm{E}-01$ & $1.96 \mathrm{E}+00$ & $3.61 \mathrm{E}-01$ & $1.22 \mathrm{E}+00$ & $1.32 \mathrm{E}-02$ & $1.06 \mathrm{E}+00$ & $8.68 \mathrm{E}-01$ \\
\hline 54 & $2.89 \mathrm{E}+00$ & $7.39 \mathrm{E}-01$ & $1.91 \mathrm{E}+00$ & $3.63 \mathrm{E}-01$ & $1.21 \mathrm{E}+00$ & $1.33 \mathrm{E}-02$ & $1.17 \mathrm{E}+00$ & $3.67 \mathrm{E}-01$ \\
\hline 55 & $2.85 \mathrm{E}+00$ & $7.43 \mathrm{E}-01$ & $1.87 \mathrm{E}+00$ & $3.65 \mathrm{E}-01$ & $1.20 \overline{\mathrm{E}}+00$ & $1.34 \mathrm{E}-02$ & $1.17 \mathrm{E}+\infty 0$ & $3.66 \mathrm{E}-01$ \\
\hline 56 & $2.81 \mathrm{E}+00$ & $7.47 \mathrm{E}-01$ & $1.83 \mathrm{E}+00$ & $3.67 \mathrm{E}-01$ & $1.19 \mathrm{E}+00$ & $1.35 \mathrm{E}-02$ & $1.16 \mathrm{E}+00$ & $3.64 \mathrm{E}-01$ \\
\hline 57 & $2.77 \mathrm{E}+00$ & $7.51 \mathrm{E}-01$ & $1.79 \mathrm{E}+00$ & $3.69 \mathrm{E}-01$ & $1.18 \mathrm{E}+00$ & $1.36 \mathrm{E}-02$ & $1.16 \mathrm{E}+00$ & $3.63 \mathrm{E}-01$ \\
\hline 58 & $2.73 \mathrm{E}+00$ & $7.55 \mathrm{E}-01$ & $1.75 \mathrm{E}+00$ & $3.71 \mathrm{E}-01$ & $1.17 \mathrm{E}+00$ & $1.38 \mathrm{E}-02$ & $1.15 \mathrm{E}+00$ & $3.62 \mathrm{E}-01$ \\
\hline 59 & $2.70 \mathrm{E}+00$ & $7.59 \mathrm{E}-01$ & $1.72 \mathrm{E}+00$ & $3.73 \mathrm{E}-01$ & $1.16 \mathrm{E}+00$ & $1.39 \mathrm{E}-02$ & $1.15 \mathrm{E}+00$ & $3.60 \mathrm{E}-01$ \\
\hline 60 & $2.66 \mathrm{E}+00$ & $7.63 \mathrm{E}-01$ & $1.68 \mathrm{E}+00$ & $3.74 \mathrm{E}-01$ & $1.15 \mathrm{E}+00$ & $1.40 \mathrm{E}-02$ & $1.14 \mathrm{E}+00$ & $3.59 \mathrm{E}-01$ \\
\hline 61 & $2.63 \mathrm{E}+00$ & 7.67E-01 & $1.65 \mathrm{E}+00$ & $3.76 \mathrm{E}-01$ & $1.15 \mathrm{E}+00$ & $1.41 \mathrm{E}-02$ & $1.14 \mathrm{E}+00$ & $3.58 \mathrm{E}-01$ \\
\hline 62 & $2.60 \mathrm{E}+00$ & $7.70 \mathrm{E}-01$ & $1.62 \mathrm{E}+00$ & $3.78 \mathrm{E}-01$ & $1.14 \mathrm{E}+00$ & $1.42 \mathrm{E}-02$ & $1.14 \mathrm{E}+00$ & $3.56 \mathrm{E}-01$ \\
\hline 63 & $2.56 \mathrm{E}+00$ & $7.74 \mathrm{E}-01$ & $1.60 \mathrm{E}+00$ & $3.79 \mathrm{E}-01$ & $1.13 \mathrm{E}+00$ & $1.43 \mathrm{E}-02$ & $1.13 \mathrm{E}+00$ & $3.55 \mathrm{E}-01$ \\
\hline 64 & $2.53 \mathrm{E}+00$ & $7.77 \mathrm{E}-01$ & $1.57 \mathrm{E}+00$ & $3.81 \mathrm{E}-01$ & $1.12 \mathrm{E}+00$ & $1.44 \mathrm{E}-02$ & $1.13 \mathrm{E}+00$ & $3.54 \mathrm{E}-01$ \\
\hline 65 & $2.50 \mathrm{E}+00$ & $7.81 \mathrm{E}-01$ & $1.54 \mathrm{E}+00$ & $3.83 \mathrm{E}-01$ & $1.11 \mathrm{E}+00$ & $1.46 \mathrm{E}-02$ & $1.12 \mathrm{E}+00$ & $3.52 \mathrm{E}-01$ \\
\hline 66 & $2.47 \mathrm{E}+00$ & $7.84 \mathrm{E}-01$ & $1.52 \mathrm{E}+00$ & $3.84 \mathrm{E}-01$ & $1.13 \mathrm{E}+00$ & $1.51 \mathrm{E}-02$ & $1.09 \mathrm{E}+00$ & $9.30 \mathrm{E}-0$ \\
\hline 67 & $2.45 \mathrm{E}+00$ & $7.88 \mathrm{E}-01$ & $1.50 \mathrm{E}+00$ & $3.86 \mathrm{E}-01$ & $1.14 \mathrm{E}+00$ & $1.57 \mathrm{E}-02$ & $1.10 \mathrm{E}+00$ & $9.33 \mathrm{E}-01$ \\
\hline 68 & $2.42 \mathrm{E}+00$ & $7.91 \mathrm{E}-01$ & $1.47 \mathrm{E}+00$ & $3.88 \mathrm{E}-01$ & $1.16 \mathrm{E}+00$ & $1.63 \mathrm{E}-02$ & $1.11 \mathrm{E}+00$ & $9.36 \mathrm{E}-01$ \\
\hline 69 & $2.39 \mathrm{E}+00$ & $7.94 \mathrm{E}-01$ & $1.45 \mathrm{E}+00$ & $3.89 \mathrm{E}-01$ & $1.17 \mathrm{E}+00$ & $1.69 \mathrm{E}-02$ & $1.11 \mathrm{E}+00$ & $9.39 \mathrm{E}-0$ \\
\hline 168 & $3.97 \mathrm{E}-01$ & $8.58 \mathrm{E}-01$ & $4.48 \mathrm{E}-01$ & $4.74 \mathrm{E}-01$ & 9.52E-01 & $7.47 \mathrm{E}-02$ & $1.05 \mathrm{E}+00$ & $9.65 \mathrm{E}-0$ \\
\hline$\overline{336}$ & $2.13 \mathrm{E}-01$ & $7.23 \mathrm{E}-01$ & $2.38 \mathrm{E}-01$ & $5.08 \mathrm{E}-01$ & $4.81 \mathrm{E}-01$ & $1.47 \mathrm{E}-01$ & $6.69 \mathrm{E}-01$ & $7.63 \mathrm{E}-01$ \\
\hline 504 & $1.57 \mathrm{E}-01$ & $6.02 \mathrm{E}-01$ & $1.64 \mathrm{E}-01$ & $5.29 \mathrm{E}-01$ & $2.68 \mathrm{E}-01$ & $1.89 \mathrm{E}-01$ & $3.51 \mathrm{E}-01$ & $5.48 \mathrm{E}-01$ \\
\hline 720 & $1.15 E-01$ & $4.74 \mathrm{E}-01$ & $1.10 \mathrm{E}-01$ & $5.48 \mathrm{E}-01$ & $1.47 \mathrm{E}-01$ & $2.14 \mathrm{E}-01$ & $1.94 \mathrm{E}-01$ & $3.92 \mathrm{E}-0$ \\
\hline 2160 & $2.15 \mathrm{E}-02$ & $9.33 \mathrm{E}-02$ & $1.24 \mathrm{E}-02$ & $5.84 \mathrm{E}-01$ & $1.32 \mathrm{E}-02$ & $1.30 \mathrm{E}-01$ & $4.69 \mathrm{E}-02$ & $1.35 \mathrm{E}-01$ \\
\hline 4320 & $2.06 \mathrm{E}-03$ & $8.32 \mathrm{E}-03$ & $6.24 \mathrm{E}-02$ & $5.53 \mathrm{E}-01$ & $2.16 \mathrm{E}-03$ & $3.11 \mathrm{E}-02$ & $5.57 \mathrm{E}-03$ & $2.08 \mathrm{E}-02$ \\
\hline 4800 & $1.23 \mathrm{E}-03$ & $4.89 \mathrm{E}-03$ & $6.22 \mathrm{E}-02$ & $5.52 \mathrm{E}-01$ & $1.57 \mathrm{E}-03$ & $2.23 \mathrm{E}-02$ & $1.84 \mathrm{E}-03$ & $6.75 \mathrm{E}-03$ \\
\hline 8760 & $1.69 \mathrm{E}-05$ & $6.29 \mathrm{E}-05$ & $6.06 \mathrm{E}-02$ & $5.38 \mathrm{E}-01$ & $1.28 \mathrm{E}-04$ & $1.49 \mathrm{E}-03$ & $5.73 \mathrm{E}-04$ & $1.98 \mathrm{E}-03$ \\
\hline$\overline{\text { DII }}$ & $\begin{array}{l}2.4 \mathrm{E} 6 \\
\mathrm{pCi} / \mathrm{L}\end{array}$ & $\begin{array}{l}8.88 \mathrm{E}+4 \\
\mathrm{~Bq} / \mathrm{kg}\end{array}$ & $88.8 \mathrm{~Bq} / \mathrm{g}$ & & & & & \\
\hline DIL HTO & $\begin{array}{l}5.9 \mathrm{E} 6 \\
\mathrm{pCi} / \mathrm{L}\end{array}$ & $\begin{array}{l}2.18 \mathrm{E}+5 \\
\mathrm{~Bq} / \mathrm{kg}\end{array}$ & $218.3 \mathrm{~Bq} / \mathrm{g}$ & & & & & \\
\hline & & & & & & & & \\
\hline & \multicolumn{2}{|c|}{ VEGETABLES } & \multicolumn{2}{|c|}{ WHEAT SEED } & \multicolumn{2}{|l|}{ MEAT } & \multicolumn{2}{|l|}{ MILK } \\
\hline \multirow[t]{2}{*}{ HOUR } & HTO & OBT & HTO & $\mathrm{OBT}$ & HTO & OBT & HTO & OBT \\
\hline & $(\mathrm{Bq} / \mathrm{g})$ & $(\mathrm{Bq} / \mathrm{g})$ & $(\mathrm{Bq} / \mathrm{g})$ & $(\mathrm{Bq} / \mathrm{g})$ & $(\mathrm{Bq} / \mathrm{g})$ & $(B q / g)$ & $(\mathrm{Bq} / \mathrm{g})$ & $(\mathrm{Bq} / \mathrm{g})$ \\
\hline
\end{tabular}




\begin{tabular}{|c|c|c|c|c|c|c|c|c|}
\hline Peak HTO & & & & & & & & \\
\hline $\begin{array}{l}12 / 13 / 29 / 3 \\
2\end{array}$ & $2.71 \mathrm{E}+01$ & $3.57 \mathrm{E}-01$ & $2.57 \mathrm{E}+01$ & $1.62 \mathrm{E}-01$ & $9.88 \mathrm{E}-01$ & $5.35 \mathrm{E}-03$ & $9.47 \mathrm{E}-01$ & $2.96 \mathrm{E}-01$ \\
\hline SUM & $2.75 E+01$ & & $2.58 \mathrm{E}+01$ & & $9.94 \mathrm{E}-01$ & & $1.24 \mathrm{E}+00$ & \\
\hline $\mathrm{Ci} / \mathrm{g}$ & $7.43 \mathrm{E}-10$ & & $6.98 \mathrm{E}-10$ & & $2.69 \mathrm{E}-11$ & & $3.36 \mathrm{E}-11$ & \\
\hline DIL HTO & $5.9 \mathrm{E}-09$ & & & & & & & \\
\hline$(\mathrm{Ci} / \mathrm{g})$ & & & & & & & & \\
\hline 168 & $3.97 \mathrm{E}-01$ & $8.58 \mathrm{E}-01$ & $4.48 \mathrm{E}-01$ & $4.74 \mathrm{E}-01$ & $9.52 \mathrm{E}-01$ & $7.47 \mathrm{E}-02$ & $1.05 \mathrm{E}+00$ & $9.65 \mathrm{E}-01$ \\
\hline SUM & $1.25 \mathrm{E}+00$ & & $9.22 \mathrm{E}-01$ & & $1.03 \mathrm{E}+00$ & & $2.01 \mathrm{E}+00$ & \\
\hline$\overline{\mathrm{Ci} / \mathrm{g}}$ & $3.39 \mathrm{E}-11$ & & $2.49 \mathrm{E}-11$ & & $2.77 \mathrm{E}-11$ & & $5.45 \mathrm{E}-11$ & \\
\hline & & . & & & & & & \\
\hline DIL OBT & $2.4 \mathrm{E}-09$ & & & & & & & \\
\hline $\mathrm{Ci} / \mathrm{g}$ & & & & & & & & \\
\hline & & & & & & & & \\
\hline 8760 & $1.69 \mathrm{E}-05$ & $6.29 \mathrm{E}-05$ & $6.06 \mathrm{E}-02$ & $5.38 \mathrm{E}-01$ & $1.28 \mathrm{E}-04$ & $1.49 \mathrm{E}-03$ & $5.73 \mathrm{E}-04$ & $1.98 \mathrm{E}-03$ \\
\hline SUM & $7.98 \mathrm{E}-05$ & & $5.98 \mathrm{E}-01$ & & $1.61 \mathrm{E}-03$ & & $2.56 \mathrm{E}-03$ & \\
\hline $\mathrm{Ci} / \mathrm{g}$ & $2.16 \mathrm{E}-15$ & & $1.62 \mathrm{E}-11$ & & $4.36 \mathrm{E}-14$ & & $6.91 \mathrm{E}-14$ & \\
\hline DIL OBT & $2.4 \mathrm{E}-09$ & & & & & & & \\
\hline $\mathrm{Ci} / \mathrm{g}$ & & & & & & & & \\
\hline & & & & & & & & \\
\hline
\end{tabular}




\begin{tabular}{|c|c|c|c|c|c|c|c|c|}
\hline & \multicolumn{6}{|c|}{ SRTC Release Scenario 1-RD-3, Adverse Met Conditions } & & \\
\hline & \multicolumn{4}{|c|}{ Concentration Values for $670 \mathrm{~m}$ from release } & & & & \\
\hline \multicolumn{9}{|c|}{ Only Wheat Seed is examined since this is the edible portion of the Wheat. The "WHEAT" category is } \\
\hline \multicolumn{9}{|c|}{ ctually the unedible plant body and is not considered in the dose estimate. } \\
\hline & & & & & & & & \\
\hline & \multicolumn{2}{|c|}{ VEGETABLES } & \multicolumn{2}{|c|}{ WHEAT SEED } & \multicolumn{2}{|l|}{ MEAT } & \multicolumn{2}{|l|}{ MILK } \\
\hline \multirow[t]{2}{*}{ HOUR } & HTO & OBT & HTO & OBT & HTO & OBT & HTO & OBT \\
\hline & $(\mathrm{Bq} / \mathrm{g})$ & $(\mathrm{Bq} / \mathrm{g})$ & $(\mathrm{Bq} / \mathrm{g})$ & $(\mathrm{Bq} / \mathrm{g})$ & $(\mathrm{Bq} / \mathrm{g})$ & $(\mathrm{Bq} / \mathrm{g})$ & $(\mathrm{Bq} / \mathrm{g})$ & $(\mathrm{Bq} / \mathrm{g}$ \\
\hline & ins & 516 & Mn & 7215 & & & & \\
\hline 1 & $1.09 \mathrm{E}+05$ & 5.16 & $1.92 \mathrm{E}+04$ & $7.31 E+01$ & 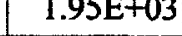 & & $\mathbf{E}+02$ & \\
\hline 2 & $4.89 \mathrm{E}+04$ & $7.27 \mathrm{E}+02$ & $2.96 \mathrm{E}+04$ & $1.34 \mathrm{E}+02$ & $2.96 \mathrm{E}+03$ & $1.04 \mathrm{E}+00$ & $5.29 \mathrm{E}+02$ & 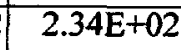 \\
\hline 3 & $2.48 \mathrm{E}+04$ & $8.20 \mathrm{E}+02$ & $3.40 \mathrm{E}+04$ & $1.84 \mathrm{E}+02$ & $3.58 \mathrm{E}+03$ & $1.81 \mathrm{E}+00$ & $8.23 E+02$ & $3.00 E+t$ \\
\hline 4 & $1.48 \mathrm{E}+04$ & $8.67 \mathrm{E}+02$ & $3.45 \mathrm{E}+04$ & $2.24 \mathrm{E}+02$ & $3.95 \mathrm{E}+03$ & $2.67 \mathrm{E}+00$ & $1.10 \mathrm{E}+03$ & 4.8 \\
\hline 5 & $1.04 \mathrm{E}+04$ & $8.95 \mathrm{E}+02$ & $3.28 \mathrm{E}+04$ & $2.55 \mathrm{E}+02$ & $4.17 \mathrm{E}+03$ & $3.59 \mathrm{E}+00$ & $1.34 \mathrm{E}+03$ & $\overline{E+0}$ \\
\hline 6 & $8.27 \mathrm{E}+03$ & $9.14 \mathrm{E}+02$ & $2.99 \mathrm{E}+04$ & $2.81 E+02$ & $4.14 \mathrm{E}+03$ & $4.03 E+00$ & $4.01 \mathrm{E}+03$ & $1.25 \mathrm{E}+0$ \\
\hline 7 & $7.15 \mathrm{E}+03$ & $9.28 \mathrm{E}+02$ & $2.65 \mathrm{E}+04$ & $3.01 E+02$ & $4.11 \mathrm{E}+03$ & $\$+00$ & $4.00 \mathrm{E}+03$ & 1.2 \\
\hline 8 & $6.49 \mathrm{E}+03$ & $9.41 \mathrm{E}+02$ & $2.31 \mathrm{E}+04$ & $3.18 \mathrm{E}+02$ & $4.08 \mathrm{E}+03$ & $E+\infty$ & $3.98 \mathrm{E}+03$ & 1.2 \\
\hline 9 & $6 . \overline{04 E}+03$ & $9.52 \mathrm{E}+02$ & $1.98 \mathrm{E}+04$ & $3.31 \mathrm{E}+02$ & $4.05 E+03$ & $5.34 \mathrm{E}+00$ & $3.97 \mathrm{E}+03$ & $1.24 \mathrm{E}+0$ \\
\hline 10 & $5.71 \mathrm{E}+03$ & $9.63 \mathrm{E}+02$ & $1.69 \mathrm{E}+04$ & $3.42 \mathrm{E}+02$ & $4.02 \mathrm{E}+03$ & 5.7 & $3.95 \mathrm{E}+03$ & 1.2 \\
\hline 11 & $5.46 \mathrm{E}+03$ & $9.73 \mathrm{E}+02$ & $1.44 \mathrm{E}+04$ & $3.51 \mathrm{E}+02$ & $3.98 \mathrm{E}+03$ & $\$+00$ & $3.94 \mathrm{E}+03$ & $1.23 \mathrm{E}+0$ \\
\hline 12 & $5.23 \mathrm{E}+03$ & $9.82 \mathrm{E}+02$ & $1.22 \mathrm{E}+04$ & $3.59 \mathrm{E}+02$ & $3.95 \mathrm{E}+03$ & $6.61 \mathrm{E}+00$ & $3.92 \mathrm{E}+03$ & $1.22 \mathrm{E}+0$ \\
\hline 13 & $5.04 \mathrm{E}+03$ & $9.91 \mathrm{E}+02$ & $1.04 \mathrm{E}+04$ & $3.65 \mathrm{E}+02$ & $3.92 E+03$ & +00 & $E+03$ & $1.22 \mathrm{E}+0$ \\
\hline 14 & $4.88 \mathrm{E}+03$ & $9.99 \mathrm{E}+02$ & $8.82 E+03$ & $3.71 \mathrm{E}+02$ & $3.89 \mathrm{E}+03$ & +00 & $3.89 \mathrm{E}+03$ & 1.2 \\
\hline 15 & $4.72 E+03$ & $1.01 \mathrm{E}+03$ & $7.54 \mathrm{E}+03$ & $3.76 E+02$ & $3.86 \mathrm{E}+03$ & $7.85 \mathrm{E}+00$ & $3.88 \mathrm{E}+03$ & $1.21 \mathrm{E}+0$ \\
\hline 16 & $4.58 \mathrm{E}+03$ & $1.02 \mathrm{E}+03$ & $6.49 \mathrm{E}+03$ & $3.80 \mathrm{E}+02$ & $3.83 \mathrm{E}+03$ & $8.26 E+00$ & $3.86 \mathrm{E}+03$ & $1.20 \mathrm{E}+$ \\
\hline 17 & $4.45 \mathrm{E}+03$ & $1.02 \mathrm{E}+03$ & $5.63 \mathrm{E}+03$ & $3.84 \mathrm{E}+02$ & $3.80 \mathrm{E}+03$ & 8.66 & $3.85 \mathrm{E}+03$ & $1.20 \mathrm{E}+\mathrm{C}$ \\
\hline 18 & $4.33 \mathrm{E}+03$ & $1.03 \mathrm{E}+03$ & $4.93 \mathrm{E}+03$ & $3.88 \mathrm{E}+02$ & $3.81 \mathrm{E}+03$ & 9.6 & $3.70 \mathrm{E}+03$ & $1.84 \mathrm{E}+0$ \\
\hline 19 & $4.21 \mathrm{E}+03$ & $1.04 \mathrm{E}+03$ & $4.36 \mathrm{E}+03$ & $3.91 \mathrm{E}+02$ & $3.83 \mathrm{E}+03$ & $1.06 \mathrm{E}+01$ & $3.70 \mathrm{E}+03$ & $1.84 \mathrm{E}+$ \\
\hline 20 & $4.10 \mathrm{E}+03$ & $1.04 \mathrm{E}+03$ & $3.89 \mathrm{E}+03$ & $3.94 \mathrm{E}+02$ & $3.84 \mathrm{E}+03$ & +01 & $3.71 E+03$ & $1.85 \mathrm{E}+0$ \\
\hline 21 & $4.00 \mathrm{E}+03$ & $1.05 \mathrm{E}+03$ & $3.51 \mathrm{E}+03$ & $3.97 \mathrm{E}+02$ & $3.85 E+03$ & +01 & $3.71 E+03$ & $1.85 \mathrm{E}+0$ \\
\hline 22 & $3.90 \mathrm{E}+03$ & $1.06 \mathrm{E}+03$ & $3.19 \mathrm{E}+03$ & $4.00 \mathrm{E}+02$ & $3.86 \mathrm{E}+03$ & 1.3 & $3.72 \mathrm{E}+03$ & $1.85 \mathrm{E}+\mathrm{C}$ \\
\hline 23 & $3.81 \mathrm{E}+03$ & $1.06 \mathrm{E}+03$ & $2.94 \mathrm{E}+03$ & $4.03 E+02$ & $3.86 \mathrm{E}+03$ & +01 & $3.72 E+03$ & $1.86 \overline{\mathrm{E}+\mathrm{C}}$ \\
\hline 24 & $3.72 \mathrm{E}+03$ & $1.07 \mathrm{E}+03$ & $2.73 \mathrm{E}+03$ & $4.05 \mathrm{E}+02$ & $3.87 \mathrm{E}+03$ & 1.5 & $3.73 E+03$ & $1.86 \mathrm{E}+0$ \\
\hline 25 & $3.63 E+03$ & $1.07 \mathrm{E}+03$ & $2.55 \mathrm{E}+03$ & $4.07 \mathrm{E}+02$ & $3.88 E+03$ & $1.65 \mathrm{E}+01$ & $3.73 E+03$ & $1.86 \mathrm{E}+\mathrm{c}$ \\
\hline 26 & $3.55 \mathrm{E}+03$ & $1.08 \mathrm{E}+03$ & $2.40 \mathrm{E}+03$ & $4.10 \mathrm{E}+02$ & $3.88 \mathrm{E}+03$ & $1.75 \mathrm{E}+01$ & $3.73 \mathrm{E}+03$ & $1.87 \mathrm{E}+\mathrm{C}$ \\
\hline 27 & $3.47 \mathrm{E}+03$ & $1.09 \mathrm{E}+03$ & $2.28 \mathrm{E}+03$ & $4.12 E+02$ & $3.89 E+03$ & $1.85 \mathrm{E}+01$ & $3.74 E+03$ & $1.87 \mathrm{E}+0$ \\
\hline 28 & $3.39 \mathrm{E}+03$ & $1.09 \mathrm{E}+03$ & $2.17 \mathrm{E}+03$ & $4.14 \mathrm{E}+02$ & $3.89 \mathrm{E}+03$ & $1.95 \mathrm{E}+01$ & $3.74 \mathrm{E}+03$ & $1.87 \overline{\mathrm{E}+\mathrm{C}}$ \\
\hline 29 & $3.32 \mathrm{E}+03$ & $1.10 \mathrm{E}+03$ & $2.08 \mathrm{E}+03$ & $4.16 \mathrm{E}+02$ & $3.89 \mathrm{E}+03$ & $2.05 \mathrm{E}+01$ & $3.74 \mathrm{E}+03$ & $1.87 \mathrm{E}+\mathrm{C}$ \\
\hline 30 & $3.24 \mathrm{E}+03$ & $1.10 \mathrm{E}+03$ & $2.00 \mathrm{E}+03$ & $4.18 \mathrm{E}+02$ & $3.86 \mathrm{E}+03$ & $2.09 \mathrm{E}+01$ & $3.74 \mathrm{E}+03$ & $1.17 \mathrm{E}+0$ \\
\hline 31 & $3.17 \mathrm{E}+03$ & $1.11 \mathrm{E}+03$ & $1.93 \bar{E}+03$ & $4.20 \mathrm{E}+02$ & $3.83 E+03$ & $2.13 \mathrm{E}+01$ & $3.73 E+03$ & $1.16 \mathrm{E}+0$ \\
\hline 32 & $3.11 \mathrm{E}+03$ & $1.11 \mathrm{E}+03$ & $1.87 \mathrm{E}+03$ & $4.22 \mathrm{E}+02$ & $3.80 \mathrm{E}+03$ & $2.17 \mathrm{E}+01$ & $3.71 \mathrm{E}+03$ & $1.16 \mathrm{E}+0$ \\
\hline 33 & $3.04 \mathrm{E}+03$ & $1.12 \mathrm{E}+03$ & $1.81 E+03$ & $4.24 \mathrm{E}+02$ & $3.77 \mathrm{E}+03$ & $2.21 \mathrm{E}+01$ & $3.70 \mathrm{E}+03$ & $1.16 \mathrm{E}+0$ \\
\hline 34 & $2.98 \mathrm{E}+03$ & $1.12 \mathrm{E}+03$ & $1.76 \mathrm{E}+03$ & $4.26 \mathrm{E}+02$ & $3.74 \mathrm{E}+03$ & $2.25 \mathrm{E}+01$ & $3.68 \mathrm{E}+03$ & $1.15 \mathrm{E}+0$ \\
\hline 35 & $2.91 \mathrm{E}+03$ & $1.12 \mathrm{E}+03$ & $1.72 E+03$ & $4.27 E+02$ & $3.70 \mathrm{E}+03$ & $2.29 E+01$ & $3.67 \mathrm{E}+03$ & $1.15 \mathrm{E}+0$ \\
\hline 36 & $2.85 \mathrm{E}+03$ & $1.13 \mathrm{E}+03$ & $1.68 \mathrm{E}+03$ & $4.29 \mathrm{E}+02$ & $3.67 \mathrm{E}+03$ & $2.32 \mathrm{E}+01$ & $3.65 \mathrm{E}+03$ & $1.14 \mathrm{E}+0$ \\
\hline 37 & $2.79 \mathrm{E}+03$ & $1.13 \mathrm{E}+03$ & $1.64 \mathrm{E}+03$ & $4.31 E+02$ & $3.64 \mathrm{E}+03$ & 2.36 & $3.64 \mathrm{E}+03$ & $1.14 \mathrm{E}+0$ \\
\hline 38 & $2.73 \mathrm{E}+03$ & $1.14 \mathrm{E}+03$ & $1.60 \mathrm{E}+03$ & $4.33 \mathrm{E}+02$ & $3.61 E+03$ & $2.40 \mathrm{E}+01$ & $3.62 \mathrm{E}+03$ & $1.13 \mathrm{E}+1$ \\
\hline
\end{tabular}




\begin{tabular}{|c|c|c|c|c|c|c|c|c|}
\hline 39 & $.67 \mathrm{E}+03$ & $1.14 \mathrm{E}+03$ & $1.56 \mathrm{E}+03$ & $4.34 \mathrm{E}+02$ & $3.59 \mathrm{E}+03$ & $2.44 \mathrm{E}+01$ & $3.61 \mathrm{E}+03$ & - \\
\hline 40 & $2.63 \mathrm{E}+03$ & $1.14 \mathrm{E}+03$ & $1.53 \mathrm{E}+03$ & $4.36 \mathrm{E}+02$ & $3.56 \mathrm{E}+03$ & $2.47 \mathrm{E}+01$ & $3.59 \overline{\mathrm{E}}+03$ & $.12 \mathrm{E}+0$ \\
\hline 41 & $2.58 \mathrm{E}+03$ & $15 \mathrm{E}+03$ & $50 \mathrm{E}+03$ & $37 E+02$ & $53 \mathrm{E}+03$ & $.51 \mathrm{E}+01$ & $.58 \mathrm{E}+03$ & $12 \mathrm{E}+0$ \\
\hline 42 & $2.54 \mathrm{E}+03$ & $5 \mathrm{E}+03$ & $1.47 \mathrm{E}+03$ & $4.39 \mathrm{E}+02$ & $3.52 \mathrm{E}+03$ & $2.61 \mathrm{E}+01$ & $3.41 \mathrm{E}+03$ & $.83 \mathrm{E}+\mathrm{C}$ \\
\hline 43 & $2.50 \mathrm{E}+03$ & $1.15 \mathrm{E}+03$ & $1.44 \mathrm{E}+03$ & $4.41 E+02$ & $3.52 \mathrm{E}+03$ & $2.71 E+01$ & $.41 \mathrm{E}+03$ & $.83 \mathrm{E}+0$ \\
\hline 44 & $2.46 \mathrm{E}+03$ & $16 \mathrm{E}+03$ & $42 \mathrm{E}+03$ & $4.42 \mathrm{E}+02$ & $3.51 \mathrm{E}+03$ & $2.80 \mathrm{E}+01$ & $3.41 \mathrm{E}+03$ & $.83 E+c$ \\
\hline 45 & $2.42 \mathrm{E}+03$ & $1.16 \mathrm{E}+03$ & $1.39 \mathrm{E}+03$ & $4.43 E+02$ & $3.51 \mathrm{E}+03$ & $2.90 \mathrm{E}+01$ & $3.41 \mathrm{E}+03$ & $.83 E+0$ \\
\hline 46 & $2.38 \mathrm{E}+03$ & $1.16 \mathrm{E}+03$ & $1.36 \mathrm{E}+03$ & $4.45 \mathrm{E}+02$ & $3.50 \mathrm{E}+03$ & $3.00 \mathrm{E}+01$ & $3.40 \mathrm{E}+03$ & $.83 E+0$ \\
\hline 47 & $2.35 \mathrm{E}+03$ & $1.17 \mathrm{E}+03$ & $1.34 \mathrm{E}+03$ & $4.46 \mathrm{E}+02$ & $3.50 \mathrm{E}+03$ & $3.10 \mathrm{E}+01$ & $3.40 \mathrm{E}+03$ & $.83 \mathrm{E}+\mathrm{C}$ \\
\hline 48 & $2.31 \mathrm{E}+03$ & $1.17 \mathrm{E}+03$ & $1.31 \mathrm{E}+03$ & $4.48 E+02$ & $3.49 \mathrm{E}+03$ & $3.20 \mathrm{E}+01$ & $3.40 \mathrm{E}+03$ & $1.83 \mathrm{E}+0$ \\
\hline 49 & $2.28 \mathrm{E}+03$ & $1.17 \mathrm{E}+03$ & $1.28 \mathrm{E}+03$ & $4.49 \mathrm{E}+02$ & $3.48 \mathrm{E}+03$ & $3.30 \mathrm{E}+01$ & $3.40 \mathrm{E}+03$ & $1.82 \mathrm{E}+0$ \\
\hline 50 & $2.25 \mathrm{E}+03$ & $1.18 \mathrm{E}+03$ & $1.26 \mathrm{E}+03$ & $4.50 \mathrm{E}+02$ & $3.48 \mathrm{E}+03$ & $3.39 \mathrm{E}+01$ & $3.39 \mathrm{E}+03$ & $1.82 \mathrm{E}+0$ \\
\hline 51 & $2.22 \mathrm{E}+03$ & $1.18 \mathrm{E}+03$ & $1.24 \mathrm{E}+03$ & $4.52 \mathrm{E}+02$ & $3.47 \mathrm{E}+03$ & $3.49 \mathrm{E}+01$ & $3.39 \mathrm{E}+03$ & $1.82 \mathrm{E}+0$ \\
\hline 52 & $2.18 \mathrm{E}+03$ & $1.18 \mathrm{E}+03$ & $1.21 \mathrm{E}+03$ & $4.53 \mathrm{E}+02$ & $3.46 \mathrm{E}+03$ & $3.59 \mathrm{E}+01$ & $.39 \mathrm{E}+03$ & $1.82 \mathrm{E}+0$ \\
\hline 53 & $2.16 \mathrm{E}+03$ & $1.18 \mathrm{E}+03$ & $1.19 \mathrm{E}+03$ & $4.54 \mathrm{E}+02$ & $3.45 \mathrm{E}+03$ & $3.69 \mathrm{E}+01$ & $3.38 \mathrm{E}+03$ & $1.82 \mathrm{E}+0$ \\
\hline 54 & $2.13 \mathrm{E}+03$ & $1.19 E+03$ & $1.17 \mathrm{E}+03$ & $4.56 \mathrm{E}+02$ & $3.42 \mathrm{E}+03$ & $3.72 \mathrm{E}+01$ & $3.32 \mathrm{E}+03$ & $1.04 \mathrm{E}+03$ \\
\hline 55 & $2.10 \mathrm{E}+03$ & $1.19 \mathrm{E}+03$ & $1.14 \mathrm{E}+03$ & $4.57 \mathrm{E}+02$ & $3.40 \mathrm{E}+03$ & $3.76 \mathrm{E}+01$ & $3.31 \mathrm{E}+03$ & $1.04 \mathrm{E}+0$ \\
\hline 56 & $2.07 \mathrm{E}+03$ & $1.19 \mathrm{E}+03$ & $1.12 \mathrm{E}+03$ & $4.58 \mathrm{E}+02$ & $3.37 \mathrm{E}+03$ & $3.79 \mathrm{E}+01$ & $3.29 \mathrm{E}+03$ & $1.03 \mathrm{E}+0$ \\
\hline 57 & $2.05 E+03$ & $1.19 \mathrm{E}+03$ & $1.10 \mathrm{E}+03$ & $4.59 \mathrm{E}+02$ & $3.34 \mathrm{E}+03$ & $3.82 \mathrm{E}+01$ & $3.28 \mathrm{E}+03$ & $1.03 \mathrm{E}+0$ \\
\hline 58 & $2.02 E+03$ & $1.20 \mathrm{E}+03$ & $1.08 \mathrm{E}+03$ & $4.60 \mathrm{E}+02$ & $3.31 \mathrm{E}+03$ & $3.86 \mathrm{E}+01$ & $3.26 \mathrm{E}+03$ & $1.02 \mathrm{E}+0$ \\
\hline 59 & $2.00 \mathrm{E}+03$ & $1.20 \mathrm{E}+03$ & $1.06 \mathrm{E}+03$ & $4.61 \mathrm{E}+02$ & $3.29 \mathrm{E}+03$ & $3.89 \mathrm{E}+01$ & $3.25 \mathrm{E}+03$ & $1.02 \mathrm{E}+0$ \\
\hline 60 & $1.97 \mathrm{E}+03$ & $1.20 \mathrm{E}+03$ & $1.04 \mathrm{E}+03$ & $4.62 E+02$ & $3.26 \mathrm{E}+03$ & $3.92 \mathrm{E}+01$ & $3.24 \mathrm{E}+03$ & $1.02 \mathrm{E}+0$ \\
\hline 61 & $1.95 \mathrm{E}+03$ & $1.20 \mathrm{E}+03$ & $1.02 \mathrm{E}+03$ & $4.64 \mathrm{E}+02$ & $3.23 \mathrm{E}+03$ & $3.95 \mathrm{E}+01$ & $3.22 \mathrm{E}+03$ & $1.01 \mathrm{E}+0$ \\
\hline 62 & $1.93 \mathrm{E}+03$ & $1.20 \mathrm{E}+03$ & $1.01 \mathrm{E}+03$ & $4.65 \mathrm{E}+02$ & $3.20 \mathrm{E}+03$ & $3.98 \mathrm{E}+01$ & $3.21 \mathrm{E}+03$ & $1.01 \mathrm{E}+0$ \\
\hline 63 & $1.91 \mathrm{E}+03$ & $1.21 \mathrm{E}+03$ & $9.89 \mathrm{E}+02$ & $4.66 \mathrm{E}+02$ & $3.18 \mathrm{E}+03$ & $4.02 \mathrm{E}+01$ & $3.20 \mathrm{E}+03$ & $1.00 \mathrm{E}+0$ \\
\hline 64 & $1.89 \mathrm{E}+03$ & $1.21 \mathrm{E}+03$ & $9.72 \mathrm{E}+02$ & $4.67 \mathrm{E}+02$ & $3.15 \mathrm{E}+03$ & $4.05 \mathrm{E}+01$ & $3.19 \mathrm{E}+03$ & $9.99 \mathrm{E}+0$ \\
\hline 65 & $1.88 \mathrm{E}+03$ & $1.21 \mathrm{E}+03$ & $9.57 \mathrm{E}+02$ & $4.68 \mathrm{E}+02$ & $3.13 \mathrm{E}$ & 4.08 & $3.17 \mathrm{E}+03$ & 9.951 \\
\hline 66 & $1.86 \mathrm{E}+03$ & $1.21 \mathrm{E}+03$ & $9.42 \mathrm{E}+02$ & $4.69 \mathrm{E}+02$ & $3.12 \mathrm{E}+03$ & $4.17 \mathrm{E}+01$ & $3.02 \mathrm{E}+03$ & $1.73 \mathrm{E}+0$ \\
\hline 67 & $1.85 \mathrm{E}+03$ & $1.22 \mathrm{E}+03$ & $9.28 \mathrm{E}+02$ & $4.70 \mathrm{E}+02$ & $3.11 \mathrm{E}+03$ & $4.27 \mathrm{E}+01$ & $3.02 \mathrm{E}+03$ & $1.73 \mathrm{E}+0$ \\
\hline 68 & $1.83 \mathrm{E}+03$ & $1.22 \mathrm{E}+03$ & $9.14 \mathrm{E}+02$ & $4.71 \mathrm{E}+02$ & $3.10 \mathrm{E}+03$ & $4.36 \mathrm{E}+01$ & $3.01 \mathrm{E}+03$ & $1.73 \mathrm{E}+0$ \\
\hline 69 & $1.82 \mathrm{E}+03$ & $1.22 \mathrm{E}+03$ & $9.02 \mathrm{E}+02$ & $4.72 \mathrm{E}+02$ & $3.09 \mathrm{E}+03$ & $4.46 \mathrm{E}+01$ & $3.01 E+03$ & $1.73 \mathrm{E}+0$ \\
\hline 168 & $4.81 \mathrm{E}+02$ & $1.28 \mathrm{E}+03$ & $4.07 \mathrm{E}+02$ & $5.32 \mathrm{E}+02$ & $1.78 E+03$ & $1.04 \mathrm{E}+02$ & $1.86 \mathrm{E}+03$ & $1.37 \mathrm{E}+0$ \\
\hline 336 & $2.23 \mathrm{E}+02$ & $1.06 \mathrm{E}+03$ & $1.86 \mathrm{E}+02$ & $5.60 \mathrm{E}+02$ & $6.89 \mathrm{E}+02$ & $1.97 \mathrm{E}+02$ & $1.10 \mathrm{E}+03$ & $1.03 \mathrm{E}+0$ \\
\hline 504 & $1.63 \mathrm{E}+02$ & $8.76 \mathrm{E}+02$ & $1.27 \mathrm{E}+02$ & $5.76 \mathrm{E}+02$ & $3.25 \mathrm{E}+02$ & $2.47 \mathrm{E}+02$ & $4.62 \mathrm{E}+02$ & $6.82 \mathrm{E}+0$ \\
\hline 720 & $1.19 \mathrm{E}+02$ & $6.80 \mathrm{E}+02$ & $8.47 \mathrm{E}+01$ & $5.90 \mathrm{E}+02$ & $1.63 \mathrm{E}+02$ & $2.74 \mathrm{E}+02$ & $2.24 E+02$ & $4.71 E+0$ \\
\hline 2160 & $2.22 \mathrm{E}+01$ & $1.24 \mathrm{E}+02$ & $9.50 \mathrm{E}+00$ & $6.17 \mathrm{E}+02$ & $1.46 \mathrm{E}+01$ & $1.60 \mathrm{E}+02$ & $5.18 \mathrm{E}+01$ & $1.61 \mathrm{E}+0$ \\
\hline 4320 & $2.13 \mathrm{E}+00$ & $1.01 \mathrm{E}+01$ & $4.79 \mathrm{E}+01$ & $5.91 \mathrm{E}+02$ & $2.24 \mathrm{E}+00$ & $3.78 \mathrm{E}+01$ & $5.99 \mathrm{E}+00$ & $2.46 \mathrm{E}+0$ \\
\hline 4800 & $1.27 \mathrm{E}+00$ & $5.85 \mathrm{E}+00$ & $4.77 \mathrm{E}+01$ & $5.89 \mathrm{E}+02$ & $1.61 \mathrm{E}+00$ & $2.71 \mathrm{E}+01$ & $1.90 \mathrm{E}+00$ & $7.88 \overline{\mathrm{E}+0}$ \\
\hline 8760 & $1.74 \mathrm{E}-02$ & $6.90 \mathrm{E}-02$ & $4.65 \mathrm{E}+01$ & $5.74 \mathrm{E}+02$ & $1.24 \mathrm{E}-01$ & $1.75 \mathrm{E}+00$ & 5.79E-01 & $2.28 \mathrm{E}+0$ \\
\hline $\mathbf{T}$ & $\begin{array}{l}2.4 \mathrm{E} 6 \\
\mathrm{pCi} / \mathrm{L}\end{array}$ & $\begin{array}{l}3 E+4 \\
\mathrm{~kg}\end{array}$ & $8 \mathrm{~Bq} / \mathrm{g}$ & & & & & \\
\hline $\bar{O}$ & $\begin{array}{l}\text { 5.9E6 } \\
\mathrm{pCi} / \mathrm{L}\end{array}$ & $\begin{array}{l}2.18 \mathrm{E}+5 \\
\mathrm{~Bq} / \mathrm{kg} \\
\end{array}$ & 218.3 Bq/g & & & & & \\
\hline & \multicolumn{2}{|c|}{ VEGETABLES } & \multicolumn{2}{|c|}{ WHEAT SEED } & \multicolumn{2}{|l|}{ MEAT } & \multicolumn{2}{|l|}{ MILK } \\
\hline \multirow[t]{2}{*}{ HOUR } & & OBT & 0 & $\overline{\mathrm{OBT}}$ & HTO & OBT & HTO & OBT \\
\hline & $(\mathrm{Bq} / \mathrm{g})$ & $(\mathrm{Bq} / \mathrm{g})$ & $(\mathrm{Bq} / \mathrm{g})$ & $(\mathrm{Bq} / \mathrm{g})$ & $(\mathrm{Bq} / \mathrm{g})$ & $(\mathrm{Bq} / \mathrm{g})$ & $(\mathrm{Bq} / \mathrm{g})$ & $(\mathrm{Bq} / \mathrm{g})$ \\
\hline ak HTO & & & & & & & & \\
\hline
\end{tabular}




\begin{tabular}{|c|c|c|c|c|c|c|c|c|}
\hline $1 / 4 / 5 / 6$ & $1.09 \mathrm{E}+05$ & $5.16 \mathrm{E}+02$ & $3.45 \mathrm{E}+04$ & $2.24 \mathrm{E}+02$ & $4.17 \mathrm{E}+03$ & $3.59 \overline{\mathrm{E}+00}$ & $4.01 \mathrm{E}+03$ & $1.25 \mathrm{E}+03$ \\
\hline SUM & $1.10 \mathrm{E}+05$ & & $3.47 \mathrm{E}+04$ & & $4.18 \mathrm{E}+03$ & & $5.27 \mathrm{E}+03$ & \\
\hline $\mathrm{Ci} / \mathrm{g}$ & $2.96 \mathrm{E}-06$ & & 9.38E-07 & & 1.13E-07 & & $1.42 \mathrm{E}-07$ & \\
\hline & & & & & & & & \\
\hline DIL HTO & $5.9 \mathrm{E}-09$ & & & & & & & \\
\hline \multicolumn{9}{|l|}{$(\mathrm{Ci} / \mathrm{g})$} \\
\hline & & & & & & & & \\
\hline 168 & $4.81 E+02$ & $1.28 \mathrm{E}+03$ & $4.07 \mathrm{E}+02$ & $5.32 \mathrm{E}+02$ & $1.78 E+03$ & $1.04 \mathrm{E}+02$ & $1.86 \mathrm{E}+03$ & $1.37 \mathrm{E}+03$ \\
\hline SUM & $1.76 \mathrm{E}+03$ & & $9.39 \mathrm{E}+02$ & & $1.88 \mathrm{E}+03$ & & $3.23 \mathrm{E}+03$ & \\
\hline $\mathrm{Ci} / \mathrm{g}$ & $4.76 \mathrm{E}-08$ & & $2.54 \mathrm{E}-08$ & & $5.09 \mathrm{E}-08$ & & $8.72 \mathrm{E}-08$ & \\
\hline & $2, \mathrm{~F}_{0} \mathrm{nol}$ & & & & & & & \\
\hline DIL OBT & $2.4 \mathrm{E}-09$ & & & & & & & \\
\hline \multicolumn{9}{|l|}{$\mathrm{Ci} / \mathrm{g}$} \\
\hline 8760 & $1.74 \mathrm{E}-02$ & $6.90 \mathrm{E}-02$ & $4.65 \mathrm{E}+01$ & $5.74 \mathrm{E}+02$ & $1.24 \mathrm{E}-01$ & $1.75 \mathrm{E}+00$ & $5.79 \mathrm{E}-01$ & $2.28 \mathrm{E}+00$ \\
\hline SUM & $8.64 \mathrm{E}-02$ & & $6.21 \mathrm{E}+02$ & & $1.87 \mathrm{E}+00$ & & $2.86 \mathrm{E}+00$ & \\
\hline $\mathrm{Ci} / \mathrm{g}$ & $2.33 \mathrm{E}-12$ & & $1.68 \mathrm{E}-08$ & & $5.05 \mathrm{E}-11$ & & $7.73 \mathrm{E}-11$ & \\
\hline DIL OBT & $2.4 \mathrm{E}-09$ & & & & & & & \\
\hline$\overline{\mathrm{Ci} / \mathrm{g}}$ & & & & & & & & \\
\hline & & & & & & & & \\
\hline & & & & & & & & \\
\hline
\end{tabular}




\begin{tabular}{|c|c|c|c|c|c|c|c|c|}
\hline & \multicolumn{6}{|c|}{ SRTC Release Scenario 1-RD-3, Adverse Met Conditions } & & \\
\hline & \multicolumn{4}{|c|}{ Concentration Values for $1 \mathrm{~km}$ from release } & & & & \\
\hline \multicolumn{9}{|c|}{ Only Wheat Seed is examined since this is the edible portion of the Wheat. The "WHEAT" category is } \\
\hline \multicolumn{9}{|c|}{ ctually the unedible plant body and is not considered in the dose estimate. } \\
\hline & & & & & & & & \\
\hline & \multicolumn{2}{|c|}{ VEGETABLES } & \multicolumn{2}{|c|}{ WHEAT SEED } & \multicolumn{2}{|l|}{ MEAT } & \multicolumn{2}{|l|}{ MILK } \\
\hline \multirow[t]{2}{*}{ HOUR } & HTO & OBT & HTO & OBT & HTO & OBT & HTO & OBT \\
\hline & $(\mathrm{Bq} / \mathrm{g})$ & $(\mathrm{Bq} / \mathrm{g})$ & $(\mathrm{Bq} / \mathrm{g})$ & $(\mathrm{Bq} / \mathrm{g})$ & $(\mathrm{Bq} / \mathrm{g})$ & $(\mathrm{Bq} / \mathrm{g})$ & $(\mathrm{Bq} / \mathrm{g})$ & $(\mathrm{Bq}$ \\
\hline & & & & & & & & \\
\hline 1 & $6.37 \mathrm{E}+04$ & $2.90 \mathrm{E}+02$ & $1.08 \mathrm{E}+04$ & $4.11 \mathrm{E}+01$ & $1.10 \mathrm{E}+03$ & $2.28 \mathrm{E}-01$ & $1.33 \mathrm{E}+02$ & $5.82 \mathrm{E}+0$ \\
\hline 2 & $2.98 \mathrm{E}+04$ & $4.13 E+02$ & $1.68 \mathrm{E}+04$ & $7.61 \mathrm{E}+01$ & $1.69 \mathrm{E}+03$ & $5.89 \mathrm{E}-01$ & $3.00 \mathrm{E}+02$ & $1.32 \mathrm{E}+0$ \\
\hline 3 & $1.58 \mathrm{E}+04$ & $4.70 \mathrm{E}+02$ & $1.95 \mathrm{E}+04$ & $1.05 \mathrm{E}+02$ & $2.05 \mathrm{E}+03$ & $1.03 \mathrm{E}+00$ & $4.68 \mathrm{E}+02$ & 2.0 \\
\hline 4 & $9.71 \mathrm{E}+03$ & $5.00 \mathrm{E}+02$ & $1.99 \mathrm{E}+04$ & $1.28 \mathrm{E}+02$ & $2.27 \mathrm{E}+03$ & $1.53 \mathrm{E}+00$ & $6.26 \mathrm{E}+02$ & $2.79 \mathrm{E}+$ \\
\hline 5 & $6.94 \mathrm{E}+03$ & $5.18 \mathrm{E}+02$ & $1.90 \mathrm{E}+04$ & $1.47 \mathrm{E}+02$ & $2.41 E+03$ & $2.05 \mathrm{E}+00$ & $7.69 \mathrm{E}+02$ & $3.44 \mathrm{E}+0$ \\
\hline 6 & $5.58 \mathrm{E}+03$ & $5.31 \mathrm{E}+02$ & $1.74 \mathrm{E}+04$ & $1.62 \mathrm{E}+02$ & $2.40 \mathrm{E}+03$ & $2.31 \mathrm{E}+00$ & $2.32 \mathrm{E}+03$ & $7.23 \mathrm{E}+$ \\
\hline 7 & $4.83 \mathrm{E}+03$ & $5.41 E+02$ & $1.56 \mathrm{E}+04$ & $1.74 E+02$ & $2.38 \mathrm{E}+03$ & $2.56 \mathrm{E}+00$ & $2.31 E+03$ & $7.21 \mathrm{E}+0$ \\
\hline 8 & $4.37 \mathrm{E}+03$ & $5.50 \mathrm{E}+02$ & $1.36 \mathrm{E}+04$ & $1.84 \mathrm{E}+02$ & $2.36 \overline{\mathrm{E}}+03$ & $2.82 \mathrm{E}+00$ & $2.30 \mathrm{E}+03$ & $7.18 E+0$ \\
\hline 9 & $4.06 \mathrm{E}+03$ & $5.57 \mathrm{E}+02$ & $1.18 \mathrm{E}+04$ & $1.93 \mathrm{E}+02$ & $2.34 \mathrm{E}+03$ & $3.07 \mathrm{E}+00$ & $2.30 \mathrm{E}+03$ & $7.15 \mathrm{E}+$ \\
\hline 10 & $3.83 E+03$ & $5.64 \mathrm{E}+02$ & $1.01 \mathrm{E}+04$ & $1.99 \mathrm{E}+02$ & $2.32 \mathrm{E}+03$ & $3.31 \mathrm{E}+00$ & $2.29 \mathrm{E}+03$ & $7.13 \mathrm{E}+$ \\
\hline 11 & $3.66 \mathrm{E}+03$ & $5.71 \mathrm{E}+02$ & $8.68 E+03$ & $2.05 \mathrm{E}+02$ & $2.31 \mathrm{E}+03$ & $3.56 \mathrm{E}+00$ & $2.28 \mathrm{E}+03$ & $7.10 \mathrm{E}+0$ \\
\hline 12 & $3.50 \mathrm{E}+03$ & $5.77 \mathrm{E}+02$ & $7.42 \mathrm{E}+03$ & $2.10 \mathrm{E}+02$ & $2.29 \mathrm{E}+03$ & $3.80 \mathrm{E}+00$ & $2.27 \mathrm{E}+03$ & $7.07 \mathrm{E}+\mathrm{c}$ \\
\hline 13 & $3.37 E+03$ & $5.83 \mathrm{E}+02$ & $6.35 \mathrm{E}+03$ & $2.14 \mathrm{E}+02$ & $2.27 \mathrm{E}+03$ & $4.04 \mathrm{E}+00$ & $2.26 \mathrm{E}+03$ & $7.04 \mathrm{E}+0$ \\
\hline 14 & $3.26 \mathrm{E}+03$ & $5.89 \mathrm{E}+02$ & $5.45 \mathrm{E}+03$ & $2.18 \mathrm{E}+02$ & $2.25 \mathrm{E}+03$ & $4.28 \mathrm{E}+00$ & $2.25 \mathrm{E}+03$ & $7.02 \mathrm{E}+0$ \\
\hline 15 & $3.16 \mathrm{E}+03$ & $5.95 \mathrm{E}+02$ & $4.71 \mathrm{E}+03$ & $2.21 \mathrm{E}+02$ & $2.24 \mathrm{E}+03$ & $4.52 \mathrm{E}+00$ & $2.24 \mathrm{E}+03$ & $6.99 \mathrm{E}+0$ \\
\hline 16 & $3.06 \mathrm{E}+03$ & $6.00 \mathrm{E}+02$ & $4.09 \mathrm{E}+03$ & $2.24 \mathrm{E}+02$ & $2.22 \mathrm{E}+03$ & $4.76 \mathrm{E}+00$ & $2.24 \mathrm{E}+03$ & $6.97 \mathrm{E}+\mathrm{C}$ \\
\hline 17 & $2.97 \mathrm{E}+03$ & $6.05 \mathrm{E}+02$ & $3.58 \mathrm{E}+03$ & $2.27 \mathrm{E}+02$ & $2.20 \mathrm{E}+03$ & $4.99 \mathrm{E}+00$ & $2.23 \mathrm{E}+03$ & $6.94 \mathrm{E}+0$ \\
\hline 18 & $2.89 \mathrm{E}+03$ & $6.10 \mathrm{E}+02$ & $3.17 \mathrm{E}+03$ & $2.29 \mathrm{E}+02$ & $2.21 \mathrm{E}+03$ & $5.56 \mathrm{E}+00$ & $2.14 \mathrm{E}+03$ & $1.08 \mathrm{E}+0$ \\
\hline 19 & $2.81 \mathrm{E}+03$ & $6.15 E+02$ & $2.82 E+03$ & $2.31 \mathrm{E}+02$ & $2.22 \mathrm{E}+03$ & $+\infty 0$ & $.15 \mathrm{E}+03$ & $E+($ \\
\hline 20 & $2.74 \mathrm{E}+03$ & $6.20 \mathrm{E}+02$ & $2.54 \mathrm{E}+03$ & $2.33 \mathrm{E}+02$ & $2.23 E+03$ & $6.71 E+00$ & $2.16 \mathrm{E}+03$ & $1.08 \mathrm{E}+$ \\
\hline 21 & $2.67 \mathrm{E}+03$ & $6.24 \mathrm{E}+02$ & $2.31 E+03$ & $2.35 \mathrm{E}+02$ & $2.24 \mathrm{E}+03$ & $7.29 \mathrm{E}+00$ & $2.16 \mathrm{E}+03$ & $1.09 \mathrm{E}+\mathrm{C}$ \\
\hline 22 & $2.60 \mathrm{E}+03$ & $6.28 \mathrm{E}+02$ & $2.12 \mathrm{E}+03$ & 2.37 & $2.25 \mathrm{E}+03$ & $7.87 \mathrm{E}+00$ & $2.17 \mathrm{E}+03$ & $1.09 \mathrm{E}+$ \\
\hline 23 & $2.54 \mathrm{E}+03$ & $6.33 \mathrm{E}+02$ & $1.97 \mathrm{E}+03$ & $2.39 \mathrm{E}+02$ & $2.26 \mathrm{E}+03$ & $8.45 \mathrm{E}+00$ & $2.17 \mathrm{E}+03$ & $1.09 \mathrm{E}+$ \\
\hline 24 & $2.48 \mathrm{E}+03$ & $6.37 \mathrm{E}+02$ & $1.84 E+03$ & $2.40 \mathrm{E}+02$ & $2.27 \mathrm{E}+03$ & $9.04 \mathrm{E}+00$ & $2.17 \mathrm{E}+03$ & $1.09 \mathrm{E}+$ \\
\hline 25 & $2.42 \mathrm{E}+03$ & $6 . \overline{41 E+02}$ & $1.73 \mathrm{E}+03$ & $2.42 \mathrm{E}+02$ & $2.28 \mathrm{E}+03$ & $9.63 E+00$ & $2.18 \mathrm{E}+03$ & $1.10 \mathrm{E}+\mathrm{C}$ \\
\hline 26 & $2.37 \mathrm{E}+03$ & $6.44 \mathrm{E}+02$ & $1.64 \mathrm{E}+03$ & $2.44 \mathrm{E}+02$ & $2.28 \mathrm{E}+03$ & $1.02 \mathrm{E}+01$ & $2.18 \mathrm{E}+03$ & $1.10 \mathrm{E}+\mathrm{C}$ \\
\hline 27 & $2.31 E+03$ & $6.48 \mathrm{E}+02$ & $1.56 \mathrm{E}+03$ & $2.45 \mathrm{E}+02$ & $2.29 \mathrm{E}+03$ & $1.08 \mathrm{E}+01$ & $2.19 \bar{E}+03$ & $1.10 \mathrm{E}+0$ \\
\hline 28 & $2.26 \mathrm{E}+03$ & $6.52 \mathrm{E}+02$ & $1.49 \mathrm{E}+03$ & $2.47 E+02$ & $2.29 \mathrm{E}+03$ & $1.14 \mathrm{E}+01$ & $2.19 \mathrm{E}+03$ & $1.10 \mathrm{E}+0$ \\
\hline 29 & $2.21 E+03$ & $6.55 \mathrm{E}+02$ & $1.43 \mathrm{E}+03$ & $2.48 \mathrm{E}+02$ & $2.30 \mathrm{E}+03$ & $1.20 \mathrm{E}+01$ & $2.19 \mathrm{E}+03$ & $1.11 \mathrm{E}+0$ \\
\hline 30 & $2.16 \mathrm{E}+03$ & $6.59 \mathrm{E}+02$ & $1.38 \mathrm{E}+03$ & $2.50 \mathrm{E}+02$ & $2.28 \mathrm{E}+03$ & $1.22 \mathrm{E}+01$ & $2.21 \bar{E}+03$ & $6.90 \mathrm{E}+0$ \\
\hline 31 & $2.11 \mathrm{E}+03$ & $6.62 \mathrm{E}+02$ & $1.34 \mathrm{E}+03$ & $2.51 \mathrm{E}+02$ & $2.26 \mathrm{E}+03$ & $1.25 \mathrm{E}+01$ & $2.20 \mathrm{E}+03$ & $6.87 \mathrm{E}+0$ \\
\hline 32 & $2.07 \mathrm{E}+03$ & $6.65 \mathrm{E}+02$ & $1.29 \mathrm{E}+03$ & $2.52 \mathrm{E}+02$ & $2.24 \mathrm{E}+03$ & $1.27 \mathrm{E}+01$ & $2.19 \mathrm{E}+03$ & $6.85 \mathrm{E}+0$ \\
\hline 33 & $2.02 \mathrm{E}+03$ & $6.68 \mathrm{E}+02$ & $1.26 \mathrm{E}+03$ & $2.54 \mathrm{E}+02$ & $2.22 \mathrm{E}+03$ & $1.29 \mathrm{E}+01$ & $2.18 \mathrm{E}+03$ & $6.82 \mathrm{E}+0$ \\
\hline 34 & $1.98 \mathrm{E}+03$ & $6.71 E+02$ & $1.22 \mathrm{E}+03$ & $2.55 \mathrm{E}+02$ & $2.21 \mathrm{E}+03$ & $1.32 \mathrm{E}+01$ & $2.17 \mathrm{E}+03$ & $6.79 \mathrm{E}+0$ \\
\hline 35 & $1.93 \mathrm{E}+03$ & $6.74 \mathrm{E}+02$ & $1.19 \mathrm{E}+03$ & $2.56 \mathrm{E}+02$ & $2.19 \mathrm{E}+03$ & $1.34 \mathrm{E}+01$ & $2.16 \mathrm{E}+03$ & $6.76 \mathrm{E}+02$ \\
\hline 36 & $1.89 \mathrm{E}+03$ & $6.77 \mathrm{E}+02$ & $1.17 \mathrm{E}+03$ & $2.57 \mathrm{E}+02$ & $2.17 \mathrm{E}+03$ & $1.36 \mathrm{E}+01$ & $2.16 \mathrm{E}+03$ & $6.74 \mathrm{E}+0$ \\
\hline 37 & $1.85 \mathrm{E}+03$ & $6.80 \mathrm{E}+02$ & $1.14 \mathrm{E}+03$ & $2.58 \mathrm{E}+02$ & $2.15 \mathrm{E}+03$ & $1.38 \mathrm{E}+01$ & $2.15 \mathrm{E}+03$ & $6.71 \mathrm{E}+0$ \\
\hline 38 & $1.81 \mathrm{E}+03$ & $6.83 \mathrm{E}+02$ & $1.11 \mathrm{E}+03$ & $2.60 \mathrm{E}+02$ & $2.14 \mathrm{E}+03$ & $1.41 \mathrm{E}+01$ & $2.14 \mathrm{E}+03$ & $6.68 \mathrm{E}+$ \\
\hline
\end{tabular}




\begin{tabular}{|c|c|c|c|c|c|c|c|c|}
\hline 39 & $1.77 \mathrm{E}+03$ & $6.85 \mathrm{E}+02$ & $1.09 \mathrm{E}+03$ & $2.61 \mathrm{E}+02$ & $2.12 \mathrm{E}+03$ & $1.43 \mathrm{E}+01$ & $2.13 E+03$ & $6.66 E+02$ \\
\hline 40 & $1.74 \mathrm{E}+03$ & $6.88 \mathrm{E}+02$ & $1.07 \mathrm{E}+03$ & $2.62 E+02$ & $2.10 \mathrm{E}+03$ & $1.45 \mathrm{E}+01$ & $2.12 \mathrm{E}+03$ & $6.63 \mathrm{E}+02$ \\
\hline 41 & $1.71 \mathrm{E}+03$ & $6.90 \mathrm{E}+02$ & $1.05 \mathrm{E}+03$ & $2.63 \bar{E}+02$ & $2.08 \mathrm{E}+03$ & $1.47 \mathrm{E}+01$ & $2.11 E+03$ & $6.60 \mathrm{E}+02$ \\
\hline 42 & $1.68 \mathrm{E}+03$ & $6.92 \mathrm{E}+02$ & $1.03 \mathrm{E}+03$ & $2.64 \mathrm{E}+02$ & $2.08 \mathrm{E}+03$ & $1.53 \mathrm{E}+01$ & $2.02 \mathrm{E}+03$ & $1.09 \mathrm{E}+03$ \\
\hline 43 & $1.65 \mathrm{E}+03$ & $6.95 \mathrm{E}+02$ & $1.01 \mathrm{E}+03$ & $2.65 \mathrm{E}+02$ & $2.08 \mathrm{E}+03$ & $1.59 \mathrm{E}+01$ & $2.02 \mathrm{E}+03$ & $1.09 \mathrm{E}+03$ \\
\hline 44 & $1.62 \mathrm{E}+03$ & $6.97 \mathrm{E}+02$ & $9.89 \mathrm{E}+02$ & $2.66 \mathrm{E}+02$ & $2.08 \mathrm{E}+03$ & $1.65 \mathrm{E}+01$ & $2.02 \mathrm{E}+03$ & $1.09 \mathrm{E}+03$ \\
\hline 45 & $1.60 \mathrm{E}+03$ & $6.99 \mathrm{E}+02$ & $9.71 \mathrm{E}+02$ & $2.67 \mathrm{E}+02$ & $2.08 \mathrm{E}+03$ & $1.71 \mathrm{E}+01$ & $2.02 \mathrm{E}+03$ & $1.09 \mathrm{E}+03$ \\
\hline 46 & $1.57 \mathrm{E}+03$ & $7.01 E+02$ & $9.52 \mathrm{E}+02$ & $2.68 E+02$ & $2.08 \mathrm{E}+03$ & $1.77 \mathrm{E}+01$ & $2.02 E+03$ & $1.09 \mathrm{E}+03$ \\
\hline 47 & $1.55 \mathrm{E}+03$ & $7.04 \mathrm{E}+02$ & $9.34 \mathrm{E}+02$ & $2.69 \mathrm{E}+02$ & $2.08 \mathrm{E}+03$ & $1.83 \mathrm{E}+01$ & $2.02 E+03$ & $1.09 E+03$ \\
\hline 48 & $1.52 \mathrm{E}+03$ & $7.06 \mathrm{E}+02$ & $9.16 \mathrm{E}+02$ & $2.70 \mathrm{E}+02$ & $2.07 \mathrm{E}+03$ & $1.88 \mathrm{E}+01$ & $2.02 \mathrm{E}+03$ & $1.09 \mathrm{E}+03$ \\
\hline 49 & $1.50 \mathrm{E}+03$ & $7.08 \mathrm{E}+02$ & $8.98 \mathrm{E}+02$ & $2.71 E+02$ & $2.07 \mathrm{E}+03$ & $1.94 \mathrm{E}+0 \mathrm{1}$ & $2.01 E+03$ & $1.09 \mathrm{E}+03$ \\
\hline 50 & $1.48 \mathrm{E}+03$ & $7.10 E+02$ & $8.80 \mathrm{E}+02$ & $2.72 E+02$ & $2.07 \mathrm{E}+03$ & $2.00 E+01$ & $2.01 E+03$ & $1.09 \mathrm{E}+03$ \\
\hline 51 & $1.45 \mathrm{E}+03$ & $7.11 \mathrm{E}+02$ & $8.63 \mathrm{E}+02$ & $2.73 \mathrm{E}+02$ & $2.07 \mathrm{E}+03$ & $2.06 \mathrm{E}+01$ & $2.01 \mathrm{E}+03$ & $1.09 \mathrm{E}+03$ \\
\hline 52 & $1.43 \mathrm{E}+03$ & $7.13 E+02$ & $8.46 \bar{E}+02$ & $2.74 \mathrm{E}+02$ & $2.06 \mathrm{E}+03$ & $2.12 \mathrm{E}+01$ & $2.01 E+03$ & $1.09 \mathrm{E}+03$ \\
\hline 53 & $1.41 \mathrm{E}+03$ & $7.15 \mathrm{E}+02$ & $8.29 \mathrm{E}+02$ & $2.75 E+02$ & $2.06 \mathrm{E}+03$ & $2.18 \mathrm{E}+01$ & $2.01 \mathrm{E}+03$ & $1.09 \mathrm{E}+03$ \\
\hline 54 & $1.39 \mathrm{E}+03$ & $7.17 \mathrm{E}+02$ & $8.13 \mathrm{E}+02$ & $2.76 \mathrm{E}+02$ & $2.04 \mathrm{E}+03$ & $2.20 \mathrm{E}+01$ & $1.98 \mathrm{E}+03$ & $6.21 \mathrm{E}+02$ \\
\hline 55 & $1.37 \mathrm{E}+03$ & $7.19 \mathrm{E}+02$ & $7.97 \mathrm{E}+02$ & $2.76 \mathrm{E}+02$ & $2.03 \mathrm{E}+03$ & $2.22 \mathrm{E}+01$ & $1.97 \mathrm{E}+03$ & $6.19 \mathrm{E}+02$ \\
\hline 56 & $1.35 \mathrm{E}+03$ & $7.20 \mathrm{E}+02$ & $7.81 \bar{E}+02$ & $2.77 \mathrm{E}+02$ & $2.01 \mathrm{E}+03$ & $2.24 \mathrm{E}+01$ & $1.96 \mathrm{E}+03$ & $6.16 \mathrm{E}+02$ \\
\hline 57 & $1.34 \mathrm{E}+03$ & $7.22 \mathrm{E}+02$ & $7.66 \mathrm{E}+02$ & $2.78 \mathrm{E}+02$ & $1.99 \mathrm{E}+03$ & $2.26 \mathrm{E}+01$ & $1.96 \mathrm{E}+03$ & $6.14 \mathrm{E}+02$ \\
\hline 58 & $1.32 \mathrm{E}+03$ & $7.24 \mathrm{E}+02$ & $7.51 \mathrm{E}+02$ & $2.79 E+02$ & $1.98 \mathrm{E}+03$ & $2.28 \mathrm{E}+01$ & $1.95 E+03$ & $6.11 E+02$ \\
\hline 59 & $1.30 \mathrm{E}+03$ & $7.25 E+02$ & $7.37 \mathrm{E}+02$ & $2.80 \mathrm{E}+02$ & $1.96 \mathrm{E}+03$ & $2.30 \mathrm{E}+01$ & $1.94 \mathrm{E}+03$ & $6.09 \mathrm{E}+02$ \\
\hline 60 & $1.28 \mathrm{E}+03$ & $7.27 \mathrm{E}+02$ & $7.23 E+02$ & $2.80 \mathrm{E}+02$ & $1.95 \mathrm{E}+03$ & $2.32 \mathrm{E}+01$ & $1.93 E+03$ & $6.06 \mathrm{E}+02$ \\
\hline 61 & $1.27 \mathrm{E}+03$ & $7.28 \mathrm{E}+02$ & $7.09 \mathrm{E}+02$ & $2.81 \mathrm{E}+02$ & $1.93 \mathrm{E}+03$ & $2.34 \mathrm{E}+01$ & $1.92 E+03$ & $6.04 \mathrm{E}+02$ \\
\hline 62 & $1.25 \mathrm{E}+03$ & $7.30 \mathrm{E}+02$ & $6.96 \mathrm{E}+02$ & $2.82 \mathrm{E}+02$ & $1.91 \mathrm{E}+03$ & $2.36 \mathrm{E}+01$ & $1.92 \mathrm{E}+03$ & $6.01 \mathrm{E}+02$ \\
\hline 63 & $1.24 \mathrm{E}+03$ & $7.31 \mathrm{E}+02$ & $6.84 \mathrm{E}+02$ & $2.83 E+02$ & $1.90 \mathrm{E}+03$ & $2.38 \mathrm{E}+01$ & $1.91 \mathrm{E}+03$ & $5.99 \mathrm{E}+02$ \\
\hline 64 & $1.21 \mathrm{E}+03$ & $7.33 \mathrm{E}+02$ & $6.71 \mathrm{E}+02$ & $2.83 \mathrm{E}+02$ & $1.88 \mathrm{E}+03$ & $2.39 \mathrm{E}+01$ & $1.90 \mathrm{E}+03$ & $5.96 \mathrm{E}+02$ \\
\hline 65 & $1.20 \mathrm{E}+03$ & $7.34 \mathrm{E}+02$ & $6.58 \mathrm{E}+02$ & $2.84 \mathrm{E}+02$ & $1.87 \mathrm{E}+03$ & $2.41 \mathrm{E}+01$ & $1.89 E+03$ & $5.94 \mathrm{E}+02$ \\
\hline 66 & $1.19 \mathrm{E}+03$ & $7.35 \mathrm{E}+02$ & $6.46 \mathrm{E}+02$ & $2.85 \mathrm{E}+02$ & $1.86 \mathrm{E}+03$ & $2.47 \mathrm{E}+01$ & $1.80 \mathrm{E}+03$ & $1.04 \mathrm{E}+03$ \\
\hline 67 & $1.17 \mathrm{E}+03$ & $7.37 \mathrm{E}+02$ & $6.33 \mathrm{E}+02$ & $2.85 \mathrm{E}+02$ & $1.86 \mathrm{E}+03$ & $2.53 \mathrm{E}+01$ & $1.80 \mathrm{E}+03$ & $1.04 \mathrm{E}+03$ \\
\hline 68 & $1.16 \mathrm{E}+03$ & $7.38 \mathrm{E}+02$ & $6.21 \mathrm{E}+02$ & $2.86 \mathrm{E}+02$ & $1.85 \mathrm{E}+03$ & $2.59 \mathrm{E}+01$ & $1.80 \mathrm{E}+03$ & $1.04 \mathrm{E}+03$ \\
\hline 69 & $1.15 \mathrm{E}+03$ & $7.39 \mathrm{E}+02$ & $6.10 \mathrm{E}+02$ & $2.87 \mathrm{E}+02$ & $1.85 E+03$ & $2.64 \mathrm{E}+01$ & $1.80 \mathrm{E}+03$ & $1.04 \mathrm{E}+03$ \\
\hline 168 & $2.93 \mathrm{E}+02$ & $7.77 \mathrm{E}+02$ & $2.46 \mathrm{E}+02$ & $3.24 \mathrm{E}+02$ & $1.07 \mathrm{E}+03$ & $6.26 \mathrm{E}+01$ & $1.12 \mathrm{E}+03$ & $8.26 \mathrm{E}+02$ \\
\hline 336 & $1.35 \mathrm{E}+02$ & $6.46 \mathrm{E}+02$ & $1.14 \mathrm{E}+02$ & $3.41 E+02$ & $4.15 \mathrm{E}+02$ & $1.19 \mathrm{E}+02$ & $6.63 \mathrm{E}+02$ & $6.27 \mathrm{E}+02$ \\
\hline 504 & $9.86 \mathrm{E}+01$ & $5.31 \mathrm{E}+02$ & $7.73 \mathrm{E}+01$ & $3.51 \mathrm{E}+02$ & $1.96 \mathrm{E}+02$ & $1.50 \mathrm{E}+02$ & $2.79 \mathrm{E}+02$ & $4.14 \mathrm{E}+02$ \\
\hline 720 & $7.17 \mathrm{E}+01$ & $4.12 \mathrm{E}+02$ & $5.17 \mathrm{E}+01$ & $3.59 \mathrm{E}+02$ & $9.88 \mathrm{E}+01$ & $1.66 \mathrm{E}+02$ & $1.36 \mathrm{E}+02$ & $2.86 \mathrm{E}+02$ \\
\hline 2160 & $1.34 \mathrm{E}+01$ & $7.52 \mathrm{E}+01$ & $5.79 \mathrm{E}+00$ & $3.75 \mathrm{E}+02$ & $8.84 \mathrm{E}+00$ & $9.70 \mathrm{E}+01$ & $3.15 \mathrm{E}+01$ & $9.78 \mathrm{E}+01$ \\
\hline 4320 & $1.29 E+00$ & $6.14 E+00$ & $2.92 \mathrm{E}+01$ & $3.59 \mathrm{E}+02$ & $1.35 \mathrm{E}+00$ & $2.29 \mathrm{E}+01$ & $3.63 \mathrm{E}+00$ & $1.49 \mathrm{E}+01$ \\
\hline 4800 & $7.67 \mathrm{E}-01$ & $3.55 \mathrm{E}+00$ & $2.91 \mathrm{E}+01$ & $3.58 \mathrm{E}+02$ & $9.74 \mathrm{E}-01$ & $1.64 \mathrm{E}+01$ & $1.15 \mathrm{E}+00$ & $4.78 \mathrm{E}+00$ \\
\hline 8760 & $1.05 \mathrm{E}-02$ & $4.18 \mathrm{E}-02$ & $2.84 \mathrm{E}+01$ & $3.49 \mathrm{E}+02$ & $7.51 \mathrm{E}-02$ & $1.06 \mathrm{E}+00$ & $3.50 \mathrm{E}-01$ & $1.38 \mathrm{E}+00$ \\
\hline$\overline{\text { DIL OBT }}$ & $\begin{array}{l}2.4 \mathrm{E} 6 \\
\mathrm{pCi} / \mathrm{L}\end{array}$ & $\begin{array}{l}8.88 \mathrm{E}+4 \\
\mathrm{~Bq} / \mathrm{kg}\end{array}$ & $88.8 \mathrm{~Bq} / \mathrm{g}$ & & & & & \\
\hline DIL HTO & $\begin{array}{l}5.9 \mathrm{E} 6 \\
\mathrm{pCi} / \mathrm{L}\end{array}$ & $\begin{array}{l}2.18 \mathrm{E}+5 \\
\mathrm{~Bq} / \mathrm{kg}\end{array}$ & $218.3 \mathrm{~Bq} / \mathrm{g}$ & & & & & \\
\hline & \multicolumn{2}{|c|}{ VEGETABLES } & \multicolumn{2}{|c|}{ WHEAT SEED } & \multicolumn{2}{|l|}{ MEAT } & \multicolumn{2}{|l|}{ MILK } \\
\hline \multirow[t]{2}{*}{ HOUR } & HTO & OBT & HTO & OBT & HTO & OBT & HTO & OBT \\
\hline & $(\mathrm{Bq} / \mathrm{g})$ & $(\overline{B q} / g)$ & $(\mathrm{Bq} / \mathrm{g})$ & $(\mathrm{B} q / \mathrm{g})$ & $(\mathrm{Bq} / \mathrm{g})$ & $(\mathrm{Bq} / \mathrm{g})$ & $(\mathrm{Bq} / \mathrm{g})$ & $(\mathrm{Bq} / \mathrm{g})$ \\
\hline Peak HTO & & & & & & & & \\
\hline
\end{tabular}




\begin{tabular}{|c|c|c|c|c|c|c|c|c|}
\hline $1 / 3 / 6 / 6$ & $6.37 E+04$ & $2.90 \mathrm{E}+02$ & $1.99 \mathrm{E}+04$ & $1.28 \mathrm{E}+02$ & $2.40 \mathrm{E}+03$ & $2.31 \mathrm{E}+00$ & $2.32 E+03$ & $7.23 E+02$ \\
\hline SUM & $6.39 E+04$ & & $2.00 \mathrm{E}+04$ & & $2.40 \bar{E}+03$ & & $3.04 \mathrm{E}+03$ & \\
\hline $\mathrm{Ci} / \mathrm{g}$ & $1.73 \mathrm{E}-06$ & & 5.41E-07 & & $6.48 \mathrm{E}-08$ & & $8.23 \mathrm{E}-08$ & \\
\hline & & & & & & & & \\
\hline DIL HTO & $5.9 \mathrm{E}-09$ & & & & & & & \\
\hline \multicolumn{9}{|l|}{$(\mathrm{Ci} / \mathrm{g})$} \\
\hline & & & & & & & & \\
\hline 168 & $2.93 \mathrm{E}+02$ & $7.77 \mathrm{E}+02$ & $2.46 \mathrm{E}+02$ & $3.24 \mathrm{E}+02$ & $1.07 \mathrm{E}+03$ & $6.26 \mathrm{E}+01$ & $1.12 \mathrm{E}+03$ & $8.26 \mathrm{E}+02$ \\
\hline$\overline{\text { SUM }}$ & $1.07 \mathrm{E}+03$ & & $5.69 \mathrm{E}+02$ & & $1.13 \mathrm{E}+03$ & & $1.94 \mathrm{E}+03$ & \\
\hline $\mathrm{Ci} / \mathrm{g}$ & $2.89 \mathrm{E}-08$ & & $1.54 \mathrm{E}-08$ & & $3.05 E-08$ & & $5.25 \mathrm{E}-08$ & \\
\hline DIl OBT & $2 A F_{0} 00$ & & & & & & & \\
\hline $\begin{array}{l} \\
\mathrm{Ci} / \mathrm{g}\end{array}$ & & & & & & & & \\
\hline & & - & & & & & & \\
\hline 8760 & $1.05 \mathrm{E}-02$ & $4.18 \mathrm{E}-02$ & $2.84 \mathrm{E}+01$ & $3.49 \mathrm{E}+02$ & $7.51 \mathrm{E}-02$ & $1.06 \mathrm{E}+00$ & $3.50 \mathrm{E}-01$ & $1.38 \mathrm{E}+00$ \\
\hline SUM & $5.23 \mathrm{E}-02$ & & $3.78 \mathrm{E}+02$ & & $1.13 E+00$ & & $1.73 \mathrm{E}+00$ & \\
\hline $\mathrm{Ci} / \mathrm{g}$ & $1.41 \mathrm{E}-12$ & & 1.02E-08 & & $3.06 \mathrm{E}-11$ & & $4.68 \mathrm{E}-11$ & \\
\hline & & & & & & & & \\
\hline DIL OBT & 2.4E-09 & & & & & & & \\
\hline $\mathrm{Ci} / \mathrm{g}$ & & & & & & & & \\
\hline & & & & & & & & \\
\hline
\end{tabular}




\begin{tabular}{|c|c|c|c|c|c|c|c|c|}
\hline & \multicolumn{6}{|c|}{ SRTC Release Scenario 1-RD-3, Adverse Met Conditions } & & \\
\hline & \multicolumn{5}{|c|}{ Concentration Values for $10 \mathrm{~km}$ from release } & & & \\
\hline \multicolumn{9}{|c|}{ Only Wheat Seed is examined since this is the edible portion of the Wheat. The "WHEAT" category is } \\
\hline \multicolumn{9}{|c|}{ actually the unedible plant body and is not considered in the dose estimate. } \\
\hline & & & & & & & & \\
\hline & \multicolumn{2}{|c|}{ VEGETABLES } & \multicolumn{2}{|c|}{ WHEAT SEED } & \multicolumn{2}{|l|}{ MEAT } & \multicolumn{2}{|l|}{ MILK } \\
\hline \multirow[t]{2}{*}{ HOUR } & HTO & OBT & HTO & OBT & HTO & OBT & HTO & OBT \\
\hline & $(\mathrm{Bq} / \mathrm{g})$ & $(\mathrm{Bq} / \mathrm{g})$ & $(\mathrm{Bq} / \mathrm{g})$ & $(\mathrm{Bq} / \mathrm{g})$ & $(\mathrm{Bq} / \mathrm{g})$ & $(\mathrm{Bq} /$ & $(\mathrm{Bq}$ & $\mathrm{Bq}$ \\
\hline & & & & & & & & \\
\hline 1 & $8.69 E+02$ & $0.00 \bar{E}+00$ & $0.00 \mathrm{E}+00$ & $0.00 \mathrm{E}+00$ & $9 . \overline{55 \mathrm{E}-01}$ & $1.03 \mathrm{E}-04$ & $1.16 \mathrm{E}-01$ & $3.60 \mathrm{E}$ \\
\hline 2 & $2.62 \mathrm{E}+03$ & $1.09 \overline{\mathrm{E}}+01$ & $4.04 \mathrm{E}+02$ & $1.54 \mathrm{E}+00$ & $4.16 \mathrm{E}+01$ & $8.72 \mathrm{E}-03$ & $4.59 \mathrm{E}+00$ & $1.99 \mathrm{E}$ \\
\hline 3 & $1.36 \mathrm{E}+03$ & $1.60 \mathrm{E}+01$ & $6.44 \mathrm{E}+02$ & $2.90 \mathrm{E}+00$ & $6.51 \mathrm{E}+01$ & $2.26 \mathrm{E}-02$ & $1.04 \mathrm{E}+01$ & $4.59 \mathrm{E}+\mathrm{c}$ \\
\hline 4 & $8.20 \mathrm{E}+02$ & $1.86 \mathrm{E}+01$ & $7.60 \mathrm{E}+02$ & $4.06 \mathrm{E}+00$ & $8.07 \mathrm{E}+01$ & $4.01 \mathrm{E}-02$ & $1.66 \mathrm{E}+01$ & $7.35 \mathrm{E}$ \\
\hline 5 & $5.69 \mathrm{E}+02$ & $2.02 \mathrm{E}+01$ & $7.94 \mathrm{E}+02$ & $5.04 \mathrm{E}+00$ & $9.11 \mathrm{E}+01$ & $5.99 \mathrm{E}-02$ & $2.26 \mathrm{E}+01$ & $1.01 \mathrm{E}+\mathrm{C}$ \\
\hline 6 & $4.45 \mathrm{E}+02$ & $2.12 \mathrm{E}+01$ & $7.76 \mathrm{E}+02$ & $5.85 \mathrm{E}+00$ & $9.06 \mathrm{E}+01$ & -02 & $8.78 \mathrm{E}+01$ & 2.74 \\
\hline 7 & $3.76 \mathrm{E}+02$ & $2.21 \mathrm{E}+01$ & $7.29 \mathrm{E}+02$ & $6.54 \mathrm{E}+00$ & $9.01 \mathrm{E}+01$ & $7.92 \mathrm{E}-02$ & $8.76 \mathrm{E}+01$ & $2.73 \mathrm{E}+\mathrm{C}$ \\
\hline 8 & $3.35 \mathrm{E}+02$ & $2.28 \mathrm{E}+01$ & $6.67 \mathrm{E}+02$ & $7.11 \mathrm{E}+00$ & $8.95 \mathrm{E}+01$ & $8.88 \mathrm{E}-02$ & $8.73 \mathrm{E}+01$ & $2.72 \mathrm{E}+\mathrm{C}$ \\
\hline 9 & $3.08 \mathrm{E}+02$ & $34 \mathrm{E}+01$ & $6.00 \mathrm{E}+02$ & $7.60 \mathrm{E}+00$ & $8.89 \mathrm{E}+01$ & -02 & $8.70 \bar{E}+01$ & $2.71 E+C$ \\
\hline 10 & $2.89 \mathrm{E}+02$ & $2.39 \mathrm{E}+01$ & $5.34 \mathrm{E}+02$ & $8.02 \mathrm{E}+00$ & $8.83 \mathrm{E}+01$ & -01 & $8.67 \mathrm{E}+01$ & $2.70 \mathrm{E}+\mathrm{H}$ \\
\hline 11 & $2.74 E+02$ & $2.45 \mathrm{E}+01$ & $4.73 E+02$ & $8.38 E+00$ & $8.77 E+01$ & $1.17 \mathrm{E}-01$ & $8.64 E+01$ & $2.69 \mathrm{E}+\mathrm{C}$ \\
\hline 12 & $2.62 \mathrm{E}+02$ & $2.50 \mathrm{E}+01$ & $4.18 \mathrm{E}+02$ & $8.70 \mathrm{E}+00$ & $8.71 \mathrm{E}+01$ & $1.26 \mathrm{E}-01$ & $8.62 \mathrm{E}+01$ & $2.68 \mathrm{E}+$ \\
\hline 13 & $2.51 \mathrm{E}+02$ & $2.54 \mathrm{E}+01$ & $3.70 \mathrm{E}+02$ & $8.98 \mathrm{E}+00$ & $8.65 E+01$ & $8-01$ & $8.59 \mathrm{E}+01$ & $2.68 \mathrm{E}$ \\
\hline 14 & $2.42 \mathrm{E}+02$ & $2.59 \mathrm{E}+01$ & $3.28 \mathrm{E}+02$ & $9.23 \mathrm{E}+00$ & $8.59 \mathrm{E}+01$ & $1.45 \mathrm{E}-01$ & $8.56 \mathrm{E}+01$ & $2.67 \mathrm{E}+\mathrm{C}$ \\
\hline 15 & $2.34 \mathrm{E}+02$ & $2.63 \mathrm{E}+01$ & $2.92 \mathrm{E}+02$ & $9.46 \mathrm{E}+00$ & $8.53 \mathrm{E}+01$ & $1.54 \mathrm{E}-01$ & $8.53 \mathrm{E}+01$ & $2.66 \mathrm{E}+$ \\
\hline 16 & $2.27 \mathrm{E}+02$ & $2.68 \mathrm{E}+01$ & $2.62 \mathrm{E}+02$ & $9.67 \mathrm{E}+00$ & $8.47 \mathrm{E}+01$ & $1.63 \mathrm{E}-01$ & $8.50 \mathrm{E}+01$ & $2.65 \mathrm{E}+$ \\
\hline 17 & $2.21 \mathrm{E}+02$ & $2.72 \mathrm{E}+01$ & $2.37 \mathrm{E}+02$ & $9.87 \mathrm{E}+00$ & $8.41 \mathrm{E}+01$ & $\mathrm{E}-01$ & $8.47 \mathrm{E}+01$ & $2.64 \mathrm{E}+$ \\
\hline 18 & $2.14 \mathrm{E}+02$ & $2.75 \mathrm{E}+01$ & $2.15 \mathrm{E}+02$ & $1.01 \mathrm{E}+01$ & $8.58 \mathrm{E}+01$ & $1.96 \mathrm{E}-01$ & $8.31 \mathrm{E}+01$ & $4.43 \mathrm{E}+$ \\
\hline 19 & $2.08 \mathrm{E}+02$ & $2.79 \mathrm{E}+01$ & $1.97 \mathrm{E}+02$ & $1.02 \mathrm{E}+01$ & $8.73 E+01$ & $2.21 \mathrm{E}-01$ & $8.39 \mathrm{E}+01$ & $4.47 \mathrm{E}+$ \\
\hline 20 & $2.03 E+02$ & $2.83 E+01$ & $1.82 E+02$ & $1.04 \mathrm{E}+01$ & $8.88 E+01$ & $2.46 \mathrm{E}-01$ & $8.46 \mathrm{E}+01$ & $4.50 \mathrm{E}+$ \\
\hline 21 & $1.98 \mathrm{E}+02$ & $2.86 \mathrm{E}+01$ & $1.70 \mathrm{E}+02$ & $1.05 \mathrm{E}+01$ & $9.02 \mathrm{E}+01$ & $2.71 \mathrm{E}-01$ & $8.53 \mathrm{E}+01$ & $4.54 \mathrm{E}+\mathrm{C}$ \\
\hline 22 & $1.93 \mathrm{E}+02$ & $2.90 \bar{E}+01$ & $1.59 \mathrm{E}+02$ & $1.07 \mathrm{E}+01$ & $9.16 \mathrm{E}+01$ & $2.97 \mathrm{E}-01$ & 8.60 & $4.5-5 x-1.5$ \\
\hline 23 & $1.89 \mathrm{E}+02$ & $2.93 \mathrm{E}+01$ & $1.50 \mathrm{E}+02$ & $1.08 \mathrm{E}+01$ & $9.30 \mathrm{E}+01$ & $3.23 \mathrm{E}-01$ & $8.67 \mathrm{E}+01$ & $4.61 \mathrm{E}+$ \\
\hline 24 & $1.85 \mathrm{E}+02$ & $2.97 \mathrm{E}+01$ & $1.42 \mathrm{E}+02$ & $1.10 \mathrm{E}+01$ & $9.43 \mathrm{E}+01$ & $3.49 \mathrm{E}-01$ & $8.74 \mathrm{E}+01$ & $4.64 \mathrm{E}+1$ \\
\hline 25 & $1.81 \mathrm{E}+02$ & $3.00 \mathrm{E}+01$ & $1.36 \mathrm{E}+02$ & $1.11 \mathrm{E}+01$ & $9.55 \mathrm{E}+01$ & 01 & $8.80 \mathrm{E}+01$ & $4.67 \mathrm{E}+\mathrm{C}$ \\
\hline 26 & $1.77 \mathrm{E}+02$ & $3.03 \mathrm{E}+01$ & $1.30 \mathrm{E}+02$ & $1.12 \mathrm{E}+01$ & $9.67 \mathrm{E}+01$ & $4.02 \mathrm{E}-01$ & $8.86 \mathrm{E}+01$ & $4.70 \mathrm{E}+$ \\
\hline 27 & $1.73 E+02$ & $3.06 \mathrm{E}+01$ & $1.25 \mathrm{E}+02$ & $1.14 \mathrm{E}+01$ & $9.78 \mathrm{E}+01$ & $4.29 \mathrm{E}-01$ & $8.93 \mathrm{E}+01$ & $4.73 E+C$ \\
\hline 28 & $1.69 \mathrm{E}+02$ & $3.09 \mathrm{E}+01$ & $1.21 \mathrm{E}+02$ & $1.15 \mathrm{E}+01$ & $9.88 \mathrm{E}+01$ & $\mathrm{E}-01$ & $8.98 \mathrm{E}+01$ & $4.76 \mathrm{~F}$ \\
\hline 29 & $1.65 \mathrm{E}+02$ & $3.12 \mathrm{E}+01$ & $1.17 \mathrm{E}+02$ & $1.16 \mathrm{E}+01$ & $9.98 \mathrm{E}+01$ & $4.84 \mathrm{E}-01$ & $9.04 \mathrm{E}+01$ & $4.79 \mathrm{E}+\mathrm{c}$ \\
\hline 30 & $1.62 \mathrm{E}+02$ & $3.14 \mathrm{E}+0 \mathrm{l}$ & $1.14 \mathrm{E}+02$ & $1.17 \mathrm{E}+01$ & $9.91 \mathrm{E}+01$ & $4.94 \mathrm{E}-01$ & $9.60 \mathrm{E}+01$ & $3.00 \mathrm{E}+$ \\
\hline 31 & $1.58 \mathrm{E}+02$ & $3.17 \mathrm{E}+01$ & $1.11 \mathrm{E}+02$ & $1.18 \mathrm{E}+01$ & $9.83 E+01$ & $5.05 \mathrm{E}-01$ & $9.56 \mathrm{E}+01$ & $2.99 \mathrm{E}+\mathrm{H}$ \\
\hline 32 & $1.55 \mathrm{E}+02$ & $3.20 \mathrm{E}+01$ & $1.08 \mathrm{E}+02$ & $1.19 \mathrm{E}+01$ & $9.75 \mathrm{E}+01$ & $5.15 \mathrm{E}-01$ & $9.53 \mathrm{E}+01$ & $2.98 \mathrm{E}+$ \\
\hline 33 & $1.52 \mathrm{E}+02$ & $3.22 \mathrm{E}+01$ & $1.06 \mathrm{E}+02$ & $1.21 \mathrm{E}+01$ & $9.68 \mathrm{E}+01$ & $5.25 \mathrm{E}-01$ & $9.49 \mathrm{E}+01$ & $2.97 \mathrm{E}+$ \\
\hline 34 & $1.48 \mathrm{E}+02$ & $3.25 \mathrm{E}+01$ & $1.04 \mathrm{E}+02$ & $1.22 \mathrm{E}+01$ & $9.61 \mathrm{E}+01$ & $5.35 \mathrm{E}-01$ & $9.46 \mathrm{E}+01$ & $2.95 \mathrm{E}+1$ \\
\hline 35 & $1.45 \mathrm{E}+02$ & $3.27 \mathrm{E}+01$ & $1.01 \mathrm{E}+02$ & $1.23 \mathrm{E}+01$ & $9.53 \mathrm{E}+01$ & $5.45 \bar{E}-01$ & $9.42 \mathrm{E}+01$ & $2.94 \mathrm{E}+\mathrm{C}$ \\
\hline 36 & $1.42 \mathrm{E}+02$ & $3.30 \mathrm{E}+01$ & $9.94 \mathrm{E}+01$ & $1.24 \mathrm{E}+01$ & $9.46 \mathrm{E}+01$ & $5.55 \mathrm{E}-01$ & $9.38 \mathrm{E}+01$ & $2.93 \mathrm{E}+\mathrm{C}$ \\
\hline 37 & $1.39 \mathrm{E}+02$ & $3.32 \mathrm{E}+01$ & $9.76 \mathrm{E}+01$ & $1.25 \mathrm{E}+01$ & $9.38 \mathrm{E}+01$ & $5.64 \mathrm{E}-01$ & $9.35 \mathrm{E}+01$ & $2.92 \mathrm{E}+$ \\
\hline 38 & $1.35 \mathrm{E}+02$ & $3.34 \mathrm{E}+01$ & $9.59 \mathrm{E}+01$ & $1.26 \mathrm{E}+01$ & $9.31 \mathrm{E}+01$ & $5.74 \mathrm{E}-01$ & $9.31 \mathrm{E}+01$ & $2.91 E+c$ \\
\hline
\end{tabular}




\begin{tabular}{|c|c|c|c|c|c|c|c|c|}
\hline 39 & $1.32 \mathrm{E}+02$ & $3.36 \mathrm{E}+01$ & $9.42 \mathrm{E}+01$ & $1.27 \mathrm{E}+01$ & $9.24 \mathrm{E}+01$ & $5.83 \mathrm{E}-01$ & $9.28 \mathrm{E}+01$ & 2.9 \\
\hline 40 & $1.30 \mathrm{E}+02$ & $3.38 \mathrm{E}+01$ & $9.27 \mathrm{E}+01$ & $1.28 \mathrm{E}+01$ & $9.17 \mathrm{E}+01$ & $5.93 \mathrm{E}-01$ & $9.24 \mathrm{E}+01$ & 80 \\
\hline 41 & $1.27 \mathrm{E}+02$ & $3.41 E+01$ & $9.12 \mathrm{E}+01$ & $1.29 \mathrm{E}+01$ & $9.09 \mathrm{E}+01$ & $6.02 \mathrm{E}-01$ & $9.20 \mathrm{E}+01$ & $.88 \mathrm{E}$ \\
\hline 42 & $1.25 \mathrm{E}+02$ & $3.43 \mathrm{E}+01$ & $8.98 \mathrm{E}+01$ & $1.30 \mathrm{E}+01$ & $9.15 E+01$ & $6.31 \mathrm{E}-01$ & $8.87 \mathrm{E}+01$ & $5.08 \mathrm{E}$ \\
\hline 43 & $1.23 \mathrm{E}+02$ & $3.45 \mathrm{E}+01$ & $8.84 \mathrm{E}+01$ & $1.31 \mathrm{E}+01$ & $9.21 \mathrm{E}+01$ & $6.59 \mathrm{E}-01$ & $8.90 \mathrm{E}+01$ & $.10 \mathrm{E}$ \\
\hline 44 & $1.21 \mathrm{E}+02$ & $3.46 \mathrm{E}+01$ & $8.70 \mathrm{E}+01$ & $1.32 \mathrm{E}+01$ & $9.26 \mathrm{E}+01$ & $6.88 \mathrm{E}-01$ & $8.92 \mathrm{E}+01$ & $5.11 \mathrm{E}$ \\
\hline 45 & $1.19 \mathrm{E}+02$ & $3.48 \mathrm{E}+01$ & $8.55 \mathrm{E}+01$ & $1.33 \mathrm{E}+01$ & $9.31 \mathrm{E}+01$ & $7.17 \mathrm{E}-01$ & $8.95 \mathrm{E}+01$ & $5.12 \mathrm{E}$ \\
\hline 46 & $1.17 \mathrm{E}+02$ & $3.50 \mathrm{E}+01$ & $8.40 E+01$ & $1.33 \mathrm{E}+01$ & $9.35 \mathrm{E}+01$ & $7.46 \mathrm{E}-01$ & $8.97 \mathrm{E}+01$ & $5.14 \mathrm{E}$ \\
\hline 47 & $1.15 \mathrm{E}+02$ & $3.52 \mathrm{E}+01$ & $8.25 E+01$ & $1.34 \mathrm{E}+01$ & $9.39 \mathrm{E}+01$ & $7.75 \mathrm{E}-01$ & $8.99 \mathrm{E}+01$ & $5.15 \mathrm{E}+$ \\
\hline 48 & $1.13 \mathrm{E}+02$ & $3.54 \mathrm{E}+01$ & $8.10 \mathrm{E}+01$ & $1.35 \mathrm{E}+01$ & $9.43 \mathrm{E}+01$ & $8.04 \mathrm{E}-01$ & $9.01 \mathrm{E}+01$ & 5.161 \\
\hline 49 & $1.11 \mathrm{E}+02$ & $3.55 \mathrm{E}+01$ & $7.95 \mathrm{E}+01$ & $1.36 \mathrm{E}+01$ & $9.47 \mathrm{E}+01$ & $8.33 \mathrm{E}-01$ & $9.03 E+01$ & $5.17 \mathrm{E}$ \\
\hline 50 & $1.09 \mathrm{E}+02$ & $3.57 \mathrm{E}+01$ & $7.80 \mathrm{E}+01$ & $1.37 \mathrm{E}+01$ & $9.51 \mathrm{E}+01$ & $8.62 \mathrm{E}-01$ & $9.05 \mathrm{E}+01$ & $5.18 \mathrm{E}$ \\
\hline 51 & $1.07 \mathrm{E}+02$ & $3.59 \mathrm{E}+01$ & $7.65 \mathrm{E}+01$ & $1.38 \mathrm{E}+01$ & $9.54 \mathrm{E}+01$ & $8.92 \mathrm{E}-01$ & $9.07 \mathrm{E}+01$ & 5.191 \\
\hline 52 & $1.05 \mathrm{E}+02$ & $3.60 \mathrm{E}+01$ & $7.50 \mathrm{E}+01$ & $1.39 \mathrm{E}+01$ & $9.57 \mathrm{E}+01$ & $9.21 \mathrm{E}-01$ & $9.09 \mathrm{E}+01$ & $5.20 \mathrm{E}$ \\
\hline 53 & $1.03 \mathrm{E}+02$ & $3.62 \mathrm{E}+01$ & $7.35 \mathrm{E}+01$ & $1.39 \mathrm{E}+01$ & $9.60 \mathrm{E}+01$ & $9.51 \mathrm{E}-01$ & $9.11 \mathrm{E}+01$ & $\overline{5.21 \mathrm{E}}$ \\
\hline 54 & $1.01 \mathrm{E}+02$ & $3.63 \mathrm{E}+01$ & $7.20 \mathrm{E}+01$ & $1.40 \mathrm{E}+01$ & $9.52 \mathrm{E}+01$ & $9.60 \mathrm{E}-01$ & $9.23 \mathrm{E}+01$ & 2.891 \\
\hline 55 & $9.97 \mathrm{E}+01$ & $3.65 \mathrm{E}+01$ & $7.05 \mathrm{E}+01$ & $1.41 \mathrm{E}+01$ & $9.44 \mathrm{E}+01$ & $9.70 \mathrm{E}-01$ & $9.19 \mathrm{E}+01$ & $2.88 \mathrm{E}$ \\
\hline 56 & $9.80 \mathrm{E}+01$ & $3.66 \mathrm{E}+01$ & $6.91 E+01$ & $1.42 \mathrm{E}+01$ & $9.37 \mathrm{E}+01$ & $9.79 \mathrm{E}-01$ & $9.15 \mathrm{E}+01$ & $2.87 \mathrm{E}-$ \\
\hline 57 & $9.64 \mathrm{E}+01$ & $3.68 \mathrm{E}+01$ & $6.77 \mathrm{E}+01$ & $1.42 \mathrm{E}+01$ & $9.29 \mathrm{E}+01$ & $9.88 \mathrm{E}-01$ & $9.12 \mathrm{E}+01$ & $2.86 \mathrm{E}+$ \\
\hline 58 & $9.48 \mathrm{E}+01$ & $3.69 \mathrm{E}+01$ & $6.63 \mathrm{E}+01$ & $1.43 \overline{\mathrm{E}}+01$ & $9.22 \mathrm{E}+01$ & $9.98 \mathrm{E}-01$ & $9.08 \mathrm{E}+01$ & $2.85 \mathrm{E}+$ \\
\hline 59 & $9.33 \mathrm{E}+01$ & $3.70 \mathrm{E}+01$ & $6.49 \mathrm{E}+01$ & $1.44 \mathrm{E}+01$ & $9.14 \mathrm{E}+01$ & $1.01 \mathrm{E}+00$ & $9.04 \mathrm{E}+01$ & $2.84 \mathrm{E}-$ \\
\hline 60 & $9.19 \mathrm{E}+01$ & $3.72 \mathrm{E}+01$ & $6.35 \mathrm{E}+01$ & $1.44 \mathrm{E}+01$ & $9.07 \mathrm{E}+01$ & $1.02 \mathrm{E}+00$ & $9.01 \mathrm{E}+01$ & $2.82 \mathrm{E}+$ \\
\hline 61 & $9.05 \mathrm{E}+01$ & $3.73 \mathrm{E}+01$ & $6.22 \mathrm{E}+01$ & $1.45 \mathrm{E}+01$ & $9.00 \mathrm{E}+01$ & $1.03 E+00$ & $8.97 \mathrm{E}+01$ & $2.81 E+$ \\
\hline 62 & $8.91 \mathrm{E}+01$ & $3.74 \mathrm{E}+01$ & $6.10 \mathrm{E}+01$ & $1.46 \mathrm{E}+01$ & $8.92 \mathrm{E}+01$ & $1.03 \mathrm{E}+00$ & $8.94 \mathrm{E}+01$ & $2.80 \mathrm{E}-$ \\
\hline 63 & $8.79 \mathrm{E}+01$ & $3.75 \bar{E}+01$ & $5.97 \mathrm{E}+01$ & $1.46 \mathrm{E}+01$ & $8.85 \mathrm{E}+01$ & $1.04 \mathrm{E}+00$ & $8.90 E+01$ & $2.79 \mathrm{E}+\mathrm{C}$ \\
\hline 64 & $8.65 \mathrm{E}+01$ & $3.77 \mathrm{E}+01$ & $5.85 \mathrm{E}+01$ & $1.47 \mathrm{E}+01$ & $8.78 \mathrm{E}+01$ & $1.05 \mathrm{E}+00$ & $8.87 \mathrm{E}+01$ & $2 . \overline{78 E+}$ \\
\hline 65 & $8.53 \mathrm{E}+01$ & $3.78 \mathrm{E}+01$ & $5.74 \mathrm{E}+01$ & $1.47 \mathrm{E}+01$ & $8.71 \mathrm{E}+01$ & $1.06 \mathrm{E}+00$ & $8.83 \mathrm{E}+01$ & $2.77 \mathrm{E}+$ \\
\hline 66 & $8.43 E+01$ & $3.79 \mathrm{E}+01$ & $5.62 \mathrm{E}+01$ & $1.48 \mathrm{E}+01$ & $8.72 \mathrm{E}+01$ & $1.09 \mathrm{E}+00$ & $8.46 \mathrm{E}+01$ & $5.18 \mathrm{E}+0$ \\
\hline 67 & $8.33 \mathrm{E}+01$ & $3.80 \mathrm{E}+01$ & $5.51 \mathrm{E}+01$ & $1.49 \mathrm{E}+01$ & $8.74 \mathrm{E}+01$ & $1.12 \mathrm{E}+00$ & $8.46 \mathrm{E}+01$ & $5.18 \mathrm{E}+$ \\
\hline 68 & $8.24 \mathrm{E}+01$ & $3.81 \mathrm{E}+01$ & $5.41 \mathrm{E}+01$ & $1.49 \mathrm{E}+01$ & $8.75 \mathrm{E}+01$ & $1.15 \mathrm{E}+00$ & $8.47 \mathrm{E}+01$ & $5.18 \mathrm{E}+\mathrm{C}$ \\
\hline 69 & $8.15 \mathrm{E}+01$ & $3.82 \mathrm{E}+01$ & $5.31 \mathrm{E}+01$ & $1.50 \mathrm{E}+01$ & $8.76 \mathrm{E}+01$ & $1.18 \mathrm{E}+00$ & $8.47 \mathrm{E}+01$ & $5.19 \mathrm{E}+\mathrm{C}$ \\
\hline 168 & $1.61 \mathrm{E}+01$ & $4.24 \mathrm{E}+01$ & $1.68 \mathrm{E}+01$ & $1.82 \mathrm{E}+01$ & $5.70 \mathrm{E}+01$ & $3.14 \mathrm{E}+00$ & $5.93 \mathrm{E}+01$ & $4.54 \mathrm{E}+$ \\
\hline 336 & $7.11 \mathrm{E}+00$ & $3.52 \mathrm{E}+01$ & $6.39 \mathrm{E}+00$ & $1.92 \mathrm{E}+01$ & $2.26 \mathrm{E}+01$ & $6.35 \mathrm{E}+00$ & $3.58 \mathrm{E}+01$ & $3.48 \mathrm{E}+\mathrm{C}$ \\
\hline 504 & $5.15 \mathrm{E}+00$ & $2.89 \mathrm{E}+01$ & $4.32 \mathrm{E}+00$ & $1.97 \mathrm{E}+01$ & $1.08 \mathrm{E}+01$ & $8.10 \mathrm{E}+00$ & $1.53 \mathrm{E}+01$ & $2.32 \mathrm{E}+0$ \\
\hline 720 & $3.74 \mathrm{E}+00$ & $2.24 \mathrm{E}+01$ & $2.87 \mathrm{E}+00$ & $2.02 E+01$ & $5.49 \mathrm{E}+00$ & $9.06 \mathrm{E}+00$ & $7.50 \mathrm{E}+00$ & $1.61 \mathrm{E}$ \\
\hline 2160 & $6.96 \mathrm{E}-01$ & $4.05 \mathrm{E}+00$ & $3.21 \mathrm{E}-01$ & $2.11 \mathrm{E}+01$ & $4.85 \mathrm{E}-01$ & $5.37 \mathrm{E}+00$ & $1.75 \mathrm{E}+00$ & $5.47 \mathrm{E}+$ \\
\hline 4320 & $6.68 \mathrm{E}-02$ & $3.27 \mathrm{E}-01$ & $1.62 \mathrm{E}+00$ & $2.02 \mathrm{E}+01$ & $7.27 \mathrm{E}-02$ & $1.27 \mathrm{E}+00$ & $1.97 \mathrm{E}-01$ & $8.24 \mathrm{E}-\mathrm{C}$ \\
\hline 4800 & $3.97 \mathrm{E}-02$ & $1.88 \mathrm{E}-01$ & $1.61 \mathrm{E}+00$ & $2.02 \mathrm{E}+01$ & $5.22 \mathrm{E}-02$ & $9.08 \mathrm{E}-01$ & $6.17 \mathrm{E}-02$ & $2.62 \mathrm{E}-\mathrm{C}$ \\
\hline 8760 & $5.46 \mathrm{E}-04$ & $2.19 \mathrm{E}-03$ & $1.57 \mathrm{E}+00$ & $1.96 \mathrm{E}+01$ & $3.97 \mathrm{E}-03$ & 5.81E-02 & $1.87 \mathrm{E}-02$ & $7.57 \mathrm{E}-$ \\
\hline BT & $\begin{array}{l}2.4 \mathrm{E} 6 \\
\mathrm{pCi} / \mathrm{L}\end{array}$ & $\begin{array}{l}8.88 \mathrm{E}+4 \\
\mathrm{~Bq} / \mathrm{kg}\end{array}$ & $8 \mathrm{~Bq} / \mathrm{g}$ & & & & & \\
\hline IL HTO & $\begin{array}{l}5.9 \mathrm{E} 6 \\
\mathrm{pCi} / \mathrm{L}\end{array}$ & $\begin{array}{l}2.18 \mathrm{E}+5 \\
\mathrm{~Bq} / \mathrm{kg}\end{array}$ & $218.3 \mathrm{~Bq} / \mathrm{g}$ & & & & & \\
\hline & \multicolumn{2}{|c|}{ VEGETABLES } & \multicolumn{2}{|c|}{ WHEAT SEED } & \multicolumn{2}{|l|}{ MEAT } & \multicolumn{2}{|l|}{ MILK } \\
\hline \multirow[t]{2}{*}{ HOUR } & TO & OBT & HTO & OBT & HTO & OBT & HTO & $\overline{\mathrm{DBT}}$ \\
\hline & $(\mathrm{Bq} / \mathrm{g})$ & $(\mathrm{Bq} / \mathrm{g})$ & $(\mathrm{Bq} / \mathrm{g})$ & $(\mathrm{Bq} / \mathrm{g})$ & $(\mathrm{Bq} / \mathrm{g})$ & $(\overline{B q} / \mathrm{g})$ & $(\mathrm{Bq} / \mathrm{g})$ & $(\mathrm{Bq} / \mathrm{g})$ \\
\hline 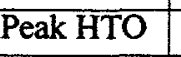 & & & & & & & & \\
\hline
\end{tabular}




\begin{tabular}{|c|c|c|c|c|c|c|c|c|}
\hline $2 / 5 / 6 / 6$ & $2.62 \mathrm{E}+03$ & $1.09 \mathrm{E}+01$ & $7.94 E+02$ & $5.04 \mathrm{E}+00$ & $9.06 \mathrm{E}+01$ & $6.96 \mathrm{E}-02$ & $8.78 \mathrm{E}+01$ & $2.74 \mathrm{E}+01$ \\
\hline$\overline{\text { SUM }}$ & $2.63 \mathrm{E}+03$ & & $7.99 \mathrm{E}+02$ & & $9.07 \mathrm{E}+01$ & & $1.15 \mathrm{E}+02$ & \\
\hline $\mathrm{Ci} / \mathrm{g}$ & $7.10 \mathrm{E}-08$ & & $2.16 E-08$ & & $2.45 \mathrm{E}-09$ & & $3.11 \mathrm{E}-09$ & \\
\hline & & & & & & & & \\
\hline DIL HTO & $5.9 \mathrm{E}-09$ & & & & & & & \\
\hline \multicolumn{9}{|l|}{$(\mathrm{Ci} / \mathrm{g})$} \\
\hline & & & & & & & & \\
\hline 168 & $1.61 \mathrm{E}+01$ & $4.24 \mathrm{E}+01$ & $1.68 \mathrm{E}+01$ & $1.82 \mathrm{E}+01$ & $5.70 \mathrm{E}+01$ & $3.14 \mathrm{E}+00$ & $5.93 \mathrm{E}+01$ & $4.54 \mathrm{E}+01$ \\
\hline SUM & $5.85 E+01$ & & $3.50 \mathrm{E}+01$ & & $6.01 E+01$ & & $1.05 E+02$ & \\
\hline $\mathrm{Ci} / \mathrm{g}$ & $1.58 \mathrm{E}-09$ & & $9.45 \mathrm{E}-10$ & & $1.62 \mathrm{E}-09$ & & $2.83 E-09$ & \\
\hline DIL OBT & $2.4 \mathrm{E}-09$ & & & & & & & \\
\hline \multicolumn{9}{|l|}{$\mathrm{Ci} / \mathrm{g}$} \\
\hline & & & & & & & & \\
\hline 8760 & $5.46 \mathrm{E}-04$ & $2.19 \mathrm{E}-03$ & $1.57 \mathrm{E}+00$ & $1.96 \mathrm{E}+01$ & $3.97 \mathrm{E}-03$ & $5.81 \mathrm{E}-02$ & $1.87 \mathrm{E}-02$ & $7.57 \mathrm{E}-02$ \\
\hline$\overline{\text { SUM }}$ & $2.73 \mathrm{E}-03$ & & $2.12 \mathrm{E}+01$ & & $6.21 \mathrm{E}-02$ & & $9.43 \mathrm{E}-02$ & \\
\hline $\mathrm{Ci} / \mathrm{g}$ & $7.38 \mathrm{E}-14$ & & $5.73 \mathrm{E}-10$ & & $1.68 \mathrm{E}-12$ & & $2.55 \mathrm{E}-12$ & \\
\hline & & & & & & & & \\
\hline DIL OBT & $2.4 \mathrm{E}-09$ & & & & & & & \\
\hline $\mathrm{Ci} / \mathrm{g}$ & & & & & & & & \\
\hline & & & & & & & & \\
\hline
\end{tabular}




\begin{tabular}{|c|c|c|c|c|c|c|c|c|}
\hline & \multicolumn{6}{|c|}{ SRTC Release Scenario 1-RD-3, Adverse Met Conditions } & & \\
\hline & \multicolumn{4}{|c|}{ Concentration Values for $50 \mathrm{~km}$ from release } & & & & \\
\hline \multicolumn{9}{|c|}{ Only Wheat Seed is examined since this is the edible portion of the Wheat. The "WHEAT" category is } \\
\hline \multicolumn{9}{|c|}{ actually the unedible plant body and is not considered in the dose estimate. } \\
\hline & & & & & & & & \\
\hline & \multicolumn{2}{|c|}{ VEGETABLES } & \multicolumn{2}{|c|}{ WHEAT SEED } & \multicolumn{2}{|l|}{ MEAT } & \multicolumn{2}{|l|}{ MILK } \\
\hline \multirow[t]{2}{*}{ HOUR } & HTO & OBT & HTO & OBT & HTO & OBT & HTO & OBT \\
\hline & $(\mathrm{Bq} / \mathrm{g})$ & $(\mathrm{Bq} / \mathrm{g})$ & $(\mathrm{Bq} / \mathrm{g})$ & $(\mathrm{Bq} / \mathrm{g})$ & $(\mathrm{Bq} / \mathrm{g})$ & $(\mathrm{Bq} / \mathrm{g})$ & $(\mathrm{Bq} / \mathrm{g})$ & $(\mathrm{Bq} / \mathrm{g})$ \\
\hline & & & & & & & & \\
\hline 1 & $2.96 \mathrm{E}+01$ & $0.00 \mathrm{E}+00$ & $0.00 \mathrm{E}+00$ & $0.00 \mathrm{E}+00$ & $3.25 \mathrm{E}-02$ & $3.49 \mathrm{E}-06$ & $3.94 \mathrm{E}-03$ & $1.23 \mathrm{E}-03$ \\
\hline 2 & $6.49 \mathrm{E}+01$ & $5.72 \mathrm{E}-02$ & $2.05 \mathrm{E}+00$ & $7.81 \mathrm{E}-03$ & $2.74 \mathrm{E}-01$ & $5.47 \mathrm{E}-05$ & $3.30 \mathrm{E}-02$ & $1.32 \mathrm{E}-02$ \\
\hline 3 & $6.68 \mathrm{E}+01$ & $1.83 \mathrm{E} \times 01$ & $6.83 E+00$ & $2.83 \mathrm{E}-02$ & $7.58 \mathrm{E}-01$ & $2.10 \mathrm{E}-04$ & $1.03 \mathrm{E}-01$ & $4.37 \mathrm{E}-02$ \\
\hline 4 & $5.97 \overline{\mathrm{E}}+01$ & $3.12 \mathrm{E}-01$ & $1.20 \mathrm{E}+01$ & $5.57 \mathrm{E}-02$ & $1.31 \mathrm{E}+00$ & $4.85 \mathrm{E}-04$ & $2.09 \mathrm{E}-01$ & $9.05 \mathrm{E}-02$ \\
\hline 5 & $7.77 \mathrm{E}+01$ & $4.27 \mathrm{E}-01$ & $1.66 \mathrm{E}+01$ & $8.64 \mathrm{E}-02$ & $1.87 \mathrm{E}+00$ & $8.82 \mathrm{E}-04$ & $3.43 \mathrm{E}-01$ & $1.50 \mathrm{E}-01$ \\
\hline 6 & $8.10 \mathrm{E}+01$ & $5.77 \mathrm{E}-01$ & $2.18 \mathrm{E}+01$ & $1.25 \mathrm{E}-01$ & $1.91 \mathrm{E}+00$ & $1.09 \mathrm{E}-03$ & $1.85 \mathrm{E}+00$ & $5.77 \mathrm{E}-01$ \\
\hline 7 & $6.95 \mathrm{E}+01$ & $7.33 \mathrm{E}-01$ & $2.69 \mathrm{E}+01$ & $1.69 \mathrm{E}-01$ & $1.94 \mathrm{E}+00$ & $1.30 \mathrm{E}-03$ & $1.87 \mathrm{E}+00$ & $5.82 \mathrm{E}-01$ \\
\hline 8 & $1.50 \mathrm{E}+02$ & $1.36 \mathrm{E}+00$ & $4.91 \mathrm{E}+01$ & $2.83 \mathrm{E}-01$ & $2.24 \mathrm{E}+00$ & $1.54 \mathrm{E}-03$ & $1.97 \mathrm{E}+00$ & $6.13 \mathrm{E}-01$ \\
\hline 9 & $3.20 \mathrm{E}+02$ & $2.92 \mathrm{E}+00$ & $1.08 \mathrm{E}+02$ & $5.61 \mathrm{E}-01$ & $2.99 \mathrm{E}+00$ & $1.86 \mathrm{E}-03$ & $2.20 \mathrm{E}+00$ & $6.85 \mathrm{E}-01$ \\
\hline 10 & $1.49 \mathrm{E}+02$ & $3.54 \mathrm{E}+00$ & $1.35 \mathrm{E}+02$ & $7.86 \mathrm{E}-01$ & $3.00 \mathrm{E}+00$ & $2.18 \mathrm{E}-03$ & $2.34 \mathrm{E}+00$ & $7.29 \mathrm{E}-01$ \\
\hline 11 & $8.73 \mathrm{E}+01$ & $3.83 \mathrm{E}+00$ & $1.43 \mathrm{E}+02$ & $9.68 \mathrm{E}-01$ & $3.01 \mathrm{E}+00$ & $2.50 \mathrm{E}-03$ & $2.44 \mathrm{E}+00$ & $7.59 \mathrm{E}-01$ \\
\hline 12 & $6.39 \mathrm{E}+01$ & $3.99 \mathrm{E}+00$ & $1.40 \mathrm{E}+02$ & $1.12 \mathrm{E}+00$ & $3.01 E+00$ & $2.82 \mathrm{E}-03$ & $2.51 \mathrm{E}+00$ & $7.81 \mathrm{E}-01$ \\
\hline 13 & $5.44 \mathrm{E}+01$ & $4.11 \mathrm{E}+00$ & $1.31 \mathrm{E}+02$ & $1.24 \mathrm{E}+00$ & $3.01 \mathrm{E}+00$ & $3.14 \mathrm{E}-03$ & $2.56 \mathrm{E}+00$ & $7.97 \mathrm{E}-01$ \\
\hline 14 & $4.99 \mathrm{E}+01$ & $4.21 \mathrm{E}+00$ & $1.19 \mathrm{E}+02$ & $1.33 \mathrm{E}+00$ & $3.01 \mathrm{E}+00$ & $3.47 \mathrm{E}-03$ & $2.60 \mathrm{E}+00$ & $8.09 \mathrm{E}-01$ \\
\hline 15 & $4.74 \mathrm{E}+01$ & $4.30 \mathrm{E}+00$ & $1.06 \mathrm{E}+02$ & $1.42 \mathrm{E}+00$ & $3.01 \mathrm{E}+00$ & $3.79 \mathrm{E}-03$ & $2.63 \mathrm{E}+00$ & $8.19 \mathrm{E}-01$ \\
\hline 16 & $4.57 \mathrm{E}+01$ & $4.39 \mathrm{E}+00$ & $9.34 \mathrm{E}+01$ & $1.49 \mathrm{E}+00$ & $3.01 \mathrm{E}+00$ & $4.11 \mathrm{E}-03$ & $2.66 \mathrm{E}+00$ & $8.28 E-01$ \\
\hline 17 & $4.45 E+01$ & $4.47 \mathrm{E}+00$ & $8.21 E+01$ & $1.55 \mathrm{E}+00$ & $3.01 E+00$ & $4.43 \mathrm{E}-03$ & $2.68 \mathrm{E}+00$ & $8.34 \mathrm{E}-01$ \\
\hline 18 & $4.34 \mathrm{E}+01$ & $4.55 \mathrm{E}+00$ & $7.21 \mathrm{E}+01$ & $1.60 \mathrm{E}+00$ & $3.48 \mathrm{E}+00$ & $7.34 \mathrm{E}-03$ & $3.37 \mathrm{E}+00$ & $4.13 E+00$ \\
\hline 19 & $4.25 \mathrm{E}+01$ & $4.63 \mathrm{E}+00$ & $6.34 \mathrm{E}+01$ & $1.65 \mathrm{E}+00$ & $3.93 E+00$ & $1.03 \mathrm{E}-02$ & $3.59 \mathrm{E}+00$ & $4.22 \mathrm{E}+00$ \\
\hline 20 & $4.16 \mathrm{E}+01$ & $4.70 \mathrm{E}+00$ & $5.61 \mathrm{E}+01$ & $1.69 \mathrm{E}+00$ & $4.36 \mathrm{E}+00$ & $1.34 \mathrm{E}-02$ & $3.80 \mathrm{E}+00$ & $4.32 \mathrm{E}+00$ \\
\hline 21 & $4.09 \mathrm{E}+01$ & $4.78 \mathrm{E}+00$ & $4.99 E+01$ & $1.73 \mathrm{E}+00$ & $4.78 \mathrm{E}+00$ & $1.66 \mathrm{E}-02$ & $4.01 \mathrm{E}+00$ & $4.41 \mathrm{E}+00$ \\
\hline 22 & $4.03 \mathrm{E}+01$ & $4.85 \mathrm{E}+00$ & $4.48 \mathrm{E}+01$ & $1.77 \mathrm{E}+00$ & $5.19 \mathrm{E}+00$ & $1.99 \mathrm{E}-02$ & $4.21 \mathrm{E}+00$ & $4.50 \mathrm{E}+00$ \\
\hline 23 & $3.99 \mathrm{E}+01$ & $4.92 \mathrm{E}+00$ & $4.06 \mathrm{E}+01$ & $1.80 \mathrm{E}+00$ & $5.59 \mathrm{E}+00$ & $2.32 \mathrm{E}-02$ & $4.42 E+00$ & $4.59 \mathrm{E}+00$ \\
\hline 24 & $3.95 \mathrm{E}+01$ & $4.99 \mathrm{E}+00$ & $3.72 \mathrm{E}+01$ & $1.83 \mathrm{E}+00$ & $5.99 \mathrm{E}+00$ & $2.66 \mathrm{E}-02$ & $4.61 E+00$ & $4.67 \mathrm{E}+00$ \\
\hline 25 & $3.91 \mathrm{E}+01$ & $5.06 \mathrm{E}+00$ & $3.44 \mathrm{E}+01$ & $1.86 \mathrm{E}+00$ & $6.37 \mathrm{E}+00$ & $3.01 \mathrm{E}-02$ & $4.81 \mathrm{E}+00$ & $4.76 \mathrm{E}+00$ \\
\hline 26 & $3.88 \mathrm{E}+01$ & $5.13 E+00$ & $3.22 \mathrm{E}+01$ & $1.89 E+00$ & $6.75 \mathrm{E}+00$ & $3.37 \mathrm{E}-02$ & $5.00 \mathrm{E}+00$ & $4.85 \mathrm{E}+00$ \\
\hline 27 & $3.85 E+01$ & $5.20 \mathrm{E}+00$ & $3.04 \mathrm{E}+01$ & $1.92 \mathrm{E}+00$ & $7.12 \mathrm{E}+00$ & $3.74 \mathrm{E}-02$ & $5.19 \mathrm{E}+00$ & $4.93 E+00$ \\
\hline 28 & $3.81 E+01$ & $5.27 \mathrm{E}+00$ & $2.89 \mathrm{E}+01$ & $1.95 \mathrm{E}+00$ & $7.48 \mathrm{E}+00$ & $4.11 \mathrm{E}-02$ & $5.38 \mathrm{E}+00$ & $5.01 \mathrm{E}+00$ \\
\hline 29 & $3.78 \mathrm{E}+01$ & $5.34 \mathrm{E}+00$ & $2.78 \mathrm{E}+01$ & $1.98 \mathrm{E}+00$ & $7.84 \mathrm{E}+00$ & $4.49 \mathrm{E}-02$ & $5.56 \mathrm{E}+00$ & $5.10 \mathrm{E}+00$ \\
\hline 30 & $3.75 \mathrm{E}+01$ & $5.40 \mathrm{E}+00$ & $2.69 \mathrm{E}+01$ & $2.01 E+00$ & $7.79 \mathrm{E}+00$ & $4.57 \mathrm{E}-02$ & $7.55 \mathrm{E}+00$ & $2.36 \mathrm{E}+00$ \\
\hline 31 & $3.72 \mathrm{E}+01$ & $5.47 \mathrm{E}+00$ & $2.62 \mathrm{E}+01$ & $2.03 \mathrm{E}+00$ & $7.74 \mathrm{E}+00$ & $4.65 \mathrm{E}-02$ & $7.53 \mathrm{E}+00$ & $2.35 \mathrm{E}+00$ \\
\hline 32 & $3.69 \mathrm{E}+01$ & $5.53 \mathrm{E}+00$ & $2.57 \mathrm{E}+01$ & $2.06 \mathrm{E}+00$ & $7.70 \mathrm{E}+00$ & 4.73E-02 & $7.50 \mathrm{E}+00$ & $2.35 \mathrm{E}+00$ \\
\hline 33 & $3.66 \mathrm{E}+01$ & $5.60 \mathrm{E}+00$ & $2.52 \mathrm{E}+01$ & $2.09 \mathrm{E}+00$ & $7.65 \mathrm{E}+00$ & $4.81 \mathrm{E}-02$ & $7.48 \mathrm{E}+00$ & $2.34 \mathrm{E}+00$ \\
\hline 34 & $3.63 E+01$ & $5.66 \mathrm{E}+00$ & $2.49 \mathrm{E}+01$ & $2.12 \mathrm{E}+00$ & $7.60 \mathrm{E}+00$ & $4.89 \overline{\mathrm{E}}-02$ & $7.46 \mathrm{E}+00$ & $2.33 \mathrm{E}+00$ \\
\hline 35 & $3.59 \mathrm{E}+01$ & $5.72 \mathrm{E}+00$ & $2.47 \mathrm{E}+01$ & $2.14 \mathrm{E}+00$ & $7.56 \mathrm{E}+00$ & $4.96 \mathrm{E}-02$ & $7.44 \mathrm{E}+00$ & $2.33 \mathrm{E}+00$ \\
\hline 36 & $3.56 \mathrm{E}+01$ & $5.78 \mathrm{E}+00$ & $2.45 \mathrm{E}+01$ & $2.17 \mathrm{E}+00$ & $7.51 \mathrm{E}+00$ & $5.04 \mathrm{E}-02$ & $7.42 \mathrm{E}+00$ & $2.32 \mathrm{E}+00$ \\
\hline 37 & $3.52 \mathrm{E}+01$ & $5.84 \mathrm{E}+00$ & $2.44 \mathrm{E}+01$ & $2.20 \mathrm{E}+00$ & $7.47 \mathrm{E}+00$ & $5.12 \mathrm{E}-02$ & $7.39 \mathrm{E}+00$ & $2.31 \mathrm{E}+00$ \\
\hline 38 & $3.48 \mathrm{E}+01$ & $5.91 \mathrm{E}+00$ & $2.43 \mathrm{E}+01$ & $2.22 \mathrm{E}+00$ & $7.42 \mathrm{E}+00$ & $5.19 \mathrm{E}-02$ & $7.37 \mathrm{E}+00$ & $2.30 \mathrm{E}+00$ \\
\hline
\end{tabular}




\begin{tabular}{|c|c|c|c|c|c|c|c|c|}
\hline 39 & $3.44 \mathrm{E}+01$ & $5.96 \mathrm{E}+00$ & $2.42 E+01$ & $2.25 \mathrm{E}+00$ & $7.38 \mathrm{E}+00$ & $5.27 \mathrm{E}-02$ & $7.35 \mathrm{E}+00$ & $2.30 \mathrm{E}+00$ \\
\hline 40 & $3.40 \mathrm{E}+01$ & $6.02 \mathrm{E}+00$ & $2.42 \mathrm{E}+01$ & $2.28 \mathrm{E}+00$ & $7.33 \mathrm{E}+00$ & $5.34 \mathrm{E}-02$ & $7.33 \mathrm{E}+00$ & $2.29 \mathrm{E}+00$ \\
\hline 41 & $3.36 \mathrm{E}+01$ & $6.08 \mathrm{E}+00$ & $2.41 \mathrm{E}+01$ & $2.30 \mathrm{E}+00$ & $7.29 \mathrm{E}+00$ & $5.42 \mathrm{E}-02$ & $7.30 \mathrm{E}+00$ & $2.28 \mathrm{E}+00$ \\
\hline 42 & $3.33 \mathrm{E}+01$ & $6.14 \mathrm{E}+00$ & $2.41 \mathrm{E}+01$ & $2.33 \mathrm{E}+00$ & $7.59 \mathrm{E}+00$ & $5.85 \mathrm{E}-02$ & $7.35 \mathrm{E}+00$ & $6.53 \mathrm{E}+00$ \\
\hline 43 & $3.29 \mathrm{E}+01$ & $6.19 \mathrm{E}+00$ & $2.41 \mathrm{E}+01$ & $2.36 \mathrm{E}+00$ & $7.88 \mathrm{E}+00$ & $6.28 \mathrm{E}-02$ & $7.50 \mathrm{E}+00$ & $6.60 \mathrm{E}+00$ \\
\hline 44 & $3.26 \mathrm{E}+01$ & $6.25 E+00$ & $2.40 \mathrm{E}+01$ & $2.38 \mathrm{E}+00$ & $8.17 \mathrm{E}+00$ & $6.72 \mathrm{E}-02$ & $7.64 \mathrm{E}+00$ & $6.66 \mathrm{E}+00$ \\
\hline 45 & $3.23 \mathrm{E}+01$ & $6.30 \mathrm{E}+00$ & $2.39 \mathrm{E}+01$ & $2.41 \mathrm{E}+00$ & $8.45 \mathrm{E}+00$ & $7.16 \mathrm{E}-02$ & $7.77 \mathrm{E}+00$ & $6.72 \bar{E}+00$ \\
\hline 46 & $3.19 \mathrm{E}+01$ & $6.36 \mathrm{E}+00$ & $2.38 \mathrm{E}+01$ & $2.44 \mathrm{E}+00$ & $8.72 \mathrm{E}+00$ & $7.61 \mathrm{E}-02$ & $7.91 \mathrm{E}+00$ & $6.78 \mathrm{E}+00$ \\
\hline 47 & $3.16 \mathrm{E}+01$ & $6.41 \mathrm{E}+00$ & $2.37 \mathrm{E}+01$ & $2.46 \mathrm{E}+00$ & $8.99 \mathrm{E}+00$ & $8.06 \mathrm{E}-02$ & $8.04 \mathrm{E}+00$ & $6.84 \mathrm{E}+00$ \\
\hline 48 & $3.12 \mathrm{E}+01$ & $6.46 \mathrm{E}+00$ & $2.35 \mathrm{E}+01$ & $2.49 \mathrm{E}+00$ & $9.25 \mathrm{E}+00$ & $8.52 \mathrm{E}-02$ & $8.18 \mathrm{E}+00$ & $6.90 \overline{\mathrm{E}}+00$ \\
\hline 49 & $3.09 \mathrm{E}+01$ & $6.52 \mathrm{E}+00$ & $2.33 \mathrm{E}+01$ & $2.51 \mathrm{E}+00$ & $9.51 \mathrm{E}+00$ & $8.99 \mathrm{E}-02$ & $8.31 \mathrm{E}+00$ & $6.95 \mathrm{E}+00$ \\
\hline 50 & $3.05 \mathrm{E}+01$ & $6.57 \mathrm{E}+00$ & $2.31 \mathrm{E}+01$ & $2.54 \mathrm{E}+00$ & $9.76 \mathrm{E}+00$ & $9.46 \mathrm{E}-02$ & $8.43 E+00$ & $7.01 E+00$ \\
\hline 51 & $3.02 \mathrm{E}+01$ & $6.62 \mathrm{E}+00$ & $2.29 \mathrm{E}+01$ & $2.56 \mathrm{E}+00$ & $1.00 \mathrm{E}+01$ & $9.93 \mathrm{E}-02$ & $8.56 \mathrm{E}+00$ & $7.06 \mathrm{E}+00$ \\
\hline 52 & $2.98 \mathrm{E}+01$ & $6.67 \mathrm{E}+00$ & $2.27 \mathrm{E}+01$ & $2.59 \mathrm{E}+00$ & $1.02 \mathrm{E}+01$ & $1.04 \mathrm{E}-01$ & $8.68 \mathrm{E}+00$ & $7.12 \mathrm{E}+00$ \\
\hline 53 & $2.94 \mathrm{E}+01$ & $6.72 \mathrm{E}+00$ & $2.24 \mathrm{E}+01$ & $2.61 \mathrm{E}+00$ & $1.05 \mathrm{E}+01$ & $1.09 \mathrm{E}-01$ & $8.81 \mathrm{E}+00$ & $7.17 \bar{E}+00$ \\
\hline 54 & $2.90 \mathrm{E}+01$ & $6.76 \mathrm{E}+00$ & $2.22 \mathrm{E}+01$ & $2.64 \mathrm{E}+00$ & $1.04 \mathrm{E}+01$ & $1.10 \mathrm{E}-01$ & $1.01 \mathrm{E}+01$ & $3.16 \mathrm{E}+00$ \\
\hline 55 & $2.86 \mathrm{E}+01$ & $6.81 E+00$ & $2.19 \mathrm{E}+01$ & $2.66 \mathrm{E}+00$ & $1.03 E+01$ & $1.11 \mathrm{E}-01$ & $1.00 \mathrm{E}+01$ & $3.15 E+00$ \\
\hline 56 & $2.82 \mathrm{E}+01$ & $6.86 \mathrm{E}+00$ & $2.17 \mathrm{E}+01$ & $2.68 \mathrm{E}+00$ & $1.03 \mathrm{E}+01$ & $1.12 \mathrm{E}-01$ & $1.00 \mathrm{E}+01$ & $3.14 \mathrm{E}+00$ \\
\hline 57 & $2.78 \mathrm{E}+01$ & $6.90 \mathrm{E}+00$ & $2.14 \mathrm{E}+01$ & $2.71 \mathrm{E}+00$ & $1.02 \mathrm{E}+01$ & $1.13 \mathrm{E}-01$ & $9.97 \mathrm{E}+00$ & $3.13 \mathrm{E}+00$ \\
\hline 58 & $2.74 \mathrm{E}+01$ & $6.95 \mathrm{E}+00$ & $2.11 \mathrm{E}+01$ & $2.73 \mathrm{E}+00$ & $1.01 \mathrm{E}+01$ & $1.14 \mathrm{E}-01$ & $9.93 E+00$ & $3.11 \mathrm{E}+00$ \\
\hline 59 & $2.70 \mathrm{E}+01$ & $6.99 \mathrm{E}+00$ & $2.08 \mathrm{E}+01$ & $2.75 \mathrm{E}+00$ & $1.00 \mathrm{E}+01$ & $1.15 \mathrm{E}-01$ & $9.90 \mathrm{E}+00$ & $3.10 \mathrm{E}+00$ \\
\hline 60 & $2.67 \mathrm{E}+01$ & $7.03 \mathrm{E}+00$ & $2.05 \mathrm{E}+01$ & $2.77 \mathrm{E}+00$ & $9.96 \mathrm{E}+00$ & $1.16 \mathrm{E}-01$ & $9.86 \mathrm{E}+00$ & $3.09 \mathrm{E}+00$ \\
\hline 61 & $2.63 \mathrm{E}+01$ & $7.08 \mathrm{E}+00$ & $2.02 E+01$ & $2.79 \mathrm{E}+00$ & $9.89 \mathrm{E}+00$ & $1.17 \mathrm{E}-01$ & $9.83 \mathrm{E}+00$ & $3.08 \mathrm{E}+00$ \\
\hline 62 & $2.59 \mathrm{E}+01$ & $7.12 \mathrm{E}+00$ & $1.99 \mathrm{E}+01$ & $2.82 \mathrm{E}+00$ & $9.81 \mathrm{E}+00$ & $1.18 \mathrm{E}-01$ & $9.79 \mathrm{E}+00$ & $3.07 \mathrm{E}+00$ \\
\hline 63 & $2.56 \mathrm{E}+01$ & $7.16 \mathrm{E}+00$ & $1.96 \mathrm{E}+01$ & $2.84 \mathrm{E}+00$ & $9.74 \mathrm{E}+00$ & $1.19 \mathrm{E}-01$ & $9.76 \mathrm{E}+00$ & $3.06 \mathrm{E}+00$ \\
\hline 64 & $2.52 \mathrm{E}+01$ & $7.20 \mathrm{E}+00$ & $1.93 \mathrm{E}+01$ & $2.86 \mathrm{E}+00$ & $9.67 \mathrm{E}+00$ & $1.20 \mathrm{E}-01$ & $9.72 \mathrm{E}+00$ & $3.05 \mathrm{E}+00$ \\
\hline 65 & $2.49 \mathrm{E}+01$ & $7.24 \mathrm{E}+00$ & $1.90 \mathrm{E}+01$ & $2.88 \mathrm{E}+00$ & $9.60 \mathrm{E}+00$ & $1.21 \mathrm{E}-01$ & $9.69 \mathrm{E}+00$ & $3.04 \bar{E}+00$ \\
\hline 66 & $2.45 \mathrm{E}+01$ & $7.28 \mathrm{E}+00$ & $1.87 \mathrm{E}+01$ & $2.90 \mathrm{E}+00$ & $9.78 \mathrm{E}+00$ & $1.26 \mathrm{E}-01$ & $9.48 \mathrm{E}+00$ & $7.97 \bar{E}+00$ \\
\hline 67 & $2.42 \mathrm{E}+01$ & $7.31 \mathrm{E}+00$ & $1.84 \mathrm{E}+01$ & $2.92 \mathrm{E}+00$ & $9.96 \mathrm{E}+00$ & $1.31 \mathrm{E}-01$ & $9.57 \mathrm{E}+00$ & $8.00 \mathrm{E}+00$ \\
\hline 68 & $2.39 \mathrm{E}+01$ & $7.35 \mathrm{E}+00$ & $1.82 \mathrm{E}+01$ & $2.94 \mathrm{E}+00$ & $1.01 \mathrm{E}+01$ & $1.36 \mathrm{E}-01$ & $9.65 \mathrm{E}+00$ & $8.04 \mathrm{E}+00$ \\
\hline 69 & $2.36 \mathrm{E}+01$ & $7.39 \mathrm{E}+00$ & $1.79 \mathrm{E}+01$ & $2.96 \mathrm{E}+00$ & $1.03 \mathrm{E}+01$ & $1.41 \mathrm{E}-01$ & $9.74 \mathrm{E}+00$ & $8.08 \mathrm{E}+00$ \\
\hline 168 & $3.43 \mathrm{E}+00$ & $8.93 E+00$ & $4.59 \mathrm{E}+00$ & $4.02 \mathrm{E}+00$ & $9.95 \mathrm{E}+00$ & $5.22 \mathrm{E}-01$ & $1.02 \mathrm{E}+01$ & $9.07 \mathrm{E}+00$ \\
\hline 336 & $1.43 \mathrm{E}+00$ & $7.41 \mathrm{E}+00$ & $1.40 \mathrm{E}+00$ & $4.24 \mathrm{E}+00$ & $4.35 \mathrm{E}+00$ & $1.23 \mathrm{E}+00$ & $6.54 \mathrm{E}+00$ & $7.23 \mathrm{E}+00$ \\
\hline 504 & $1.03 \mathrm{E}+00$ & $6.08 \mathrm{E}+00$ & $9.37 \mathrm{E}-01$ & $4.36 \mathrm{E}+00$ & $2.22 \mathrm{E}+00$ & $1.63 \mathrm{E}+00$ & $3.03 \mathrm{E}+00$ & $4.98 \mathrm{E}+00$ \\
\hline 720 & $7.43 \mathrm{E}-01$ & $4.71 \mathrm{E}+00$ & $6.21 E-01$ & $4.46 \mathrm{E}+00$ & $1.18 \mathrm{E}+00$ & $1.86 \mathrm{E}+00$ & $1.58 \mathrm{E}+00$ & $3.49 \mathrm{E}+00$ \\
\hline 2160 & $1.38 \mathrm{E}-01$ & 8.41E-01 & $6.89 \mathrm{E}-02$ & $4.65 \mathrm{E}+00$ & $1.04 \mathrm{E}-01$ & $1.13 \mathrm{E}+00$ & $3.78 \mathrm{E}-01$ & $1.19 \mathrm{E}+00$ \\
\hline 4320 & $1.32 \mathrm{E}-02$ & $6.68 \mathrm{E}-02$ & $3.49 \mathrm{E}-01$ & $4.46 \mathrm{E}+00$ & $1.52 \mathrm{E}-02$ & $2.68 \mathrm{E}-01$ & $4.17 \mathrm{E}-02$ & $1.75 \mathrm{E}-01$ \\
\hline 4800 & $7.86 \mathrm{E}-03$ & $3.84 \mathrm{E}-02$ & $3.48 \mathrm{E}-01$ & $4.45 \mathrm{E}+\infty 0$ & $1.09 \mathrm{E}-02$ & $1.92 \mathrm{E}-01$ & $1.29 \mathrm{E}-02$ & $5.53 \mathrm{E}-02$ \\
\hline 8760 & $1.08 \mathrm{E}-04$ & $4.38 \mathrm{E}-04$ & $3.39 \mathrm{E}-01$ & $4.34 \mathrm{E}+00$ & $8.21 \mathrm{E}-04$ & $1.23 \mathrm{E}-02$ & $3.87 \mathrm{E}-03$ & $1.59 \mathrm{E}-02$ \\
\hline$\overline{D I L ~ O B T}$ & $\begin{array}{l}2.4 \mathrm{E} 6 \\
\mathrm{pCi} / \mathrm{L}\end{array}$ & $\begin{array}{l}8.88 \mathrm{E}+4 \\
\mathrm{~B} / \mathrm{kg}\end{array}$ & $88.8 \mathrm{~Bq} / \mathrm{g}$ & & & & & \\
\hline DIL HTO & $\begin{array}{l}5.9 \mathrm{E} 6 \\
\mathrm{pCi} / \mathrm{L}\end{array}$ & $\begin{array}{l}2.18 \mathrm{E}+5 \\
\mathrm{~Bq} / \mathrm{kg} \\
\end{array}$ & $218.3 \mathrm{~Bq} / \mathrm{g}$ & & & & & \\
\hline & \multicolumn{2}{|c|}{ VEGETABLES } & \multicolumn{2}{|c|}{ WHEAT SEED } & \multicolumn{2}{|l|}{ MEAT } & \multicolumn{2}{|l|}{ MILK } \\
\hline \multirow[t]{2}{*}{ HOUR } & $\overline{\text { HTO }}$ & OBT & HTO & $\mathrm{OBT}$ & HTO & OBT & HTO & OBT \\
\hline & $(\mathrm{Bq} / \mathrm{g})$ & $(\mathrm{Bq} / \mathrm{g})$ & $(\mathrm{Bq} / \mathrm{g})$ & $(\mathrm{Bq} / \mathrm{g})$ & $(\mathrm{Bq} / \mathrm{g})$ & $(\mathrm{Bq} / \mathrm{g})$ & $(\mathrm{Bq} / \mathrm{g})$ & $(\mathrm{Bq} / \mathrm{g})$ \\
\hline Peak HTO & & & & & & & & \\
\hline
\end{tabular}




\begin{tabular}{|c|c|c|c|c|c|c|c|c|}
\hline $9 / 11 / 53 / 6$ & $3.20 \mathrm{E}+02$ & $2.92 E+00$ & $1.43 \mathrm{E}+02$ & $9.68 \mathrm{E}-01$ & $1.05 \mathrm{E}+01$ & $1.09 \mathrm{E}-01$ & $1.01 \mathrm{E}+01$ & $3.16 \mathrm{E}+00$ \\
\hline SUM & $3.23 \mathrm{E}+02$ & & $1.44 \mathrm{E}+02$ & & $1.06 \mathrm{E}+01$ & & $1.32 E+01$ & \\
\hline $\mathrm{Ci} / \mathrm{g}$ & $8.72 E-09$ & & $3.90 \mathrm{E}-09$ & & $2.86 \mathrm{E}-10$ & & $3.58 \mathrm{E}-10$ & \\
\hline & & & & & & & & \\
\hline DIL HTO & $5.9 \mathrm{E}-09$ & & & & & & & \\
\hline \multicolumn{9}{|l|}{$(\mathrm{Ci} / \mathrm{g})$} \\
\hline & & & & & & & & \\
\hline 168 & $3.43 \mathrm{E}+00$ & $8.93 E+00$ & $4.59 \mathrm{E}+00$ & $4.02 \mathrm{E}+00$ & $9.95 \mathrm{E}+00$ & $5.22 \mathrm{E}-01$ & $1.02 \mathrm{E}+01$ & $9.07 \mathrm{E}+00$ \\
\hline SUM & $1.24 E+01$ & & $8.60 E+00$ & & $1.05 E+01$ & & $1.93 \mathrm{E}+01$ & \\
\hline$\overline{\mathrm{Ci} / \mathrm{g}}$ & $3.34 \mathrm{E}-10$ & & $2.33 \mathrm{E}-10$ & & $2.83 \mathrm{E}-10$ & & $5.20 \mathrm{E}-10$ & \\
\hline & & & & & & & & \\
\hline DIL OBT & $2.4 \mathrm{E}-09$ & & & & & & & \\
\hline $\mathrm{Ci} / \mathrm{g}$ & & 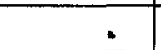 & & & & & & \\
\hline & & & & & & & & \\
\hline 8760 & $1.08 \mathrm{E}-04$ & $4.38 \mathrm{E}-04$ & 3.39E-01 & $4.34 \mathrm{E}+00$ & $8.21 \mathrm{E}-04$ & $1.23 \mathrm{E}-02$ & $3.87 \mathrm{E}-03$ & $1.59 \mathrm{E}-02$ \\
\hline SUM & $5.46 \mathrm{E}-04$ & & $4.68 \mathrm{E}+00$ & & $1.31 \mathrm{E}-02$ & & $1.98 \mathrm{E}-02$ & \\
\hline $\mathrm{Ci} / \mathrm{g}$ & $1.47 \mathrm{E}-14$ & & $1.26 \mathrm{E}-10$ & & $3.54 \mathrm{E}-13$ & & $5.35 \mathrm{E}-13$ & \\
\hline & & & & & & & & \\
\hline DIL OBT & $2.4 \mathrm{E}-09$ & & & & & & & \\
\hline $\mathrm{Ci} / \mathrm{g}$ & & & & & & & & \\
\hline & & & & & & & & \\
\hline
\end{tabular}




\begin{tabular}{|c|c|c|c|c|c|c|c|c|}
\hline & \multicolumn{6}{|c|}{ SRTC Release Scenario 1-RD-3, Adverse Met Conditions } & & \\
\hline & \multicolumn{4}{|c|}{ Concentration Values for $100 \mathrm{~km}$ from release } & & & & \\
\hline \multicolumn{9}{|c|}{ Only Wheat Seed is examined since this is the edible portion of the Wheat. The "WHEAT" category is } \\
\hline \multicolumn{9}{|c|}{ actually the unedible plant body and is not considered in the dose estimate. } \\
\hline & & & & & & & & \\
\hline & \multicolumn{2}{|c|}{ VEGETABLES } & \multicolumn{2}{|c|}{ WHEAT SEED } & \multicolumn{2}{|l|}{ MEAT } & \multicolumn{2}{|l|}{ MILK } \\
\hline \multirow[t]{2}{*}{ HOUR } & HTO & OBT & HTO & OBT & HTO & OBT & HTO & OBT \\
\hline & $(\mathrm{Bq} / \mathrm{g})$ & $(\mathrm{Bq} / \mathrm{g})$ & $(\mathrm{Bq} / \mathrm{g})$ & $(\mathrm{Bq} / \mathrm{g})$ & $(\mathrm{Bq} / \mathrm{g})$ & $(\mathrm{Bq} / \mathrm{g})$ & $(\mathrm{Bq} / \mathrm{g})$ & $(\mathrm{Bq} / \mathrm{g})$ \\
\hline & & & & & & & & \\
\hline 1 & $9.70 \mathrm{E}-01$ & $0.00 \mathrm{E}+00$ & $0.00 \mathrm{E}+00$ & $0.00 \mathrm{E}+00$ & $1.07 \mathrm{E}-03$ & $1.15 \mathrm{E}-07$ & $1.29 \mathrm{E}-04$ & 4.02E-05 \\
\hline 2 & $2.78 \mathrm{E}+00$ & $1.88 \mathrm{E}-03$ & 6.74E-02 & $2.57 \mathrm{E}-04$ & $9.72 \mathrm{E}-03$ & $1.87 \mathrm{E}-06$ & $1.16 \mathrm{E}-03$ & $4.58 \mathrm{E}-04$ \\
\hline 3 & $3.83 \mathrm{E}+00$ & $7.27 \mathrm{E}_{-} 03$ & $2.70 \mathrm{E}-01$ & $1.10 \mathrm{E}-03$ & $3.12 \mathrm{E}-02$ & $8.13 \mathrm{E}-06$ & $4.07 \mathrm{E}-03$ & $1.70 \mathrm{E}-03$ \\
\hline 4 & $4.37 \mathrm{E}+00$ & $1.47 \mathrm{E}-02$ & $5.60 \mathrm{E}-01$ & $2.51 \mathrm{E}-03$ & $6.21 \mathrm{E}-02$ & $2.10 \mathrm{E}-05$ & $9.17 \mathrm{E}-03$ & $3.94 \mathrm{E}-03$ \\
\hline 5 & $5.78 \mathrm{E}+00$ & $2.31 \mathrm{E}-02$ & $8.91 \mathrm{E}-01$ & $4.39 \mathrm{E}-03$ & $1.00 \mathrm{E}-01$ & $4.21 \mathrm{E}-05$ & $1.65 \mathrm{E}-02$ & $7.18 \mathrm{E}-03$ \\
\hline 6 & $6.89 \mathrm{E}+00$ & $3.43 \mathrm{E}-02$ & $1.31 \mathrm{E}+00$ & $6.96 \mathrm{E}-03$ & $1.05 \mathrm{E}-01$ & $5.33 \mathrm{E}-05$ & $1.02 \mathrm{E}-01$ & $3.17 \mathrm{E}-02$ \\
\hline 7 & $7.22 \mathrm{E}+00$ & $4.76 \mathrm{E}-02$ & $1.78 \mathrm{E}+00$ & $1.02 \mathrm{E}-02$ & $1.09 \mathrm{E}-01$ & $6.51 \mathrm{E}-05$ & $1.04 \mathrm{E}-01$ & $3.23 \mathrm{E}-02$ \\
\hline 8 & $8.48 \mathrm{E}+00$ & $6.15 \mathrm{E}-02$ & $2.25 \mathrm{E}+00$ & $1.40 \mathrm{E}-02$ & $1.15 \mathrm{E}-01$ & $7.74 \mathrm{E}-05$ & $1.06 \mathrm{E}-01$ & $3.31 \mathrm{E}-02$ \\
\hline 9 & $1.19 \mathrm{E}+01$ & $7.78 \mathrm{E}-02$ & $2.76 \mathrm{E}+00$ & $1.84 \mathrm{E}-02$ & $1.24 \mathrm{E}-01$ & $9.06 \mathrm{E}-05$ & $1.10 \mathrm{E}-01$ & $3.42 \mathrm{E}-02$ \\
\hline 10 & $1.24 \mathrm{E}+01$ & $1.01 \mathrm{E}-01$ & $3.48 \mathrm{E}+00$ & $2.42 \mathrm{E}-02$ & $1.31 \mathrm{E}-01$ & $1.05 \mathrm{E}-04$ & $1.13 \mathrm{E}-01$ & $3.53 \mathrm{E}-02$ \\
\hline 11 & $1.18 \mathrm{E}+01$ & $1.25 \mathrm{E}-01$ & $4.20 \mathrm{E}+00$ & $3.09 \mathrm{E}-02$ & $1.38 \mathrm{E}-01$ & $1.20 \mathrm{E}-04$ & $1.17 \mathrm{E}-01$ & $3.63 \mathrm{E}-02$ \\
\hline 12 & $1.40 \mathrm{E}+01$ & $1.47 \mathrm{E}-01$ & $4.83 \mathrm{E}+00$ & $3.79 \mathrm{E}-02$ & $1.48 \mathrm{E}-01$ & $1.35 \mathrm{E}-04$ & $1.20 \mathrm{E}-01$ & $3.75 \mathrm{E}-02$ \\
\hline 13 & $2.22 \mathrm{E}+01$ & $1.74 \mathrm{E}-01$ & $5.53 \mathrm{E}+00$ & $4.60 \mathrm{E}-02$ & $1.65 \mathrm{E}-01$ & $1.53 \mathrm{E}-04$ & $1.25 \mathrm{E}-01$ & $3.91 \mathrm{E}-02$ \\
\hline 14 & $2.07 \mathrm{E}+01$ & $2.17 \mathrm{E}-01$ & $6.75 E+00$ & $5.68 E-02$ & $1.78 \mathrm{E}-01$ & $1.72 \mathrm{E}-04$ & $1.31 \mathrm{E}-01$ & $4.07 E-02$ \\
\hline 15 & $1.79 \bar{E}+01$ & $2.57 \mathrm{E}-01$ & $7.88 \mathrm{E}+00$ & $6.86 \mathrm{E}-02$ & $1.88 \mathrm{E}-01$ & $1.92 \mathrm{E}-04$ & $1.36 \mathrm{E}-01$ & $4.23 \mathrm{E}-02$ \\
\hline 16 & $3.63 \mathrm{E}+01$ & $4.02 \mathrm{E}-01$ & $1.29 \mathrm{E}+01$ & $9.65 \mathrm{E}-02$ & $2.60 \mathrm{E}-01$ & $2.20 \mathrm{E}-04$ & $1.46 \mathrm{E}-01$ & $4.56 \mathrm{E}-02$ \\
\hline 17 & $9.01 \mathrm{E}+01$ & $8.37 \mathrm{E}-01$ & $2.93 \mathrm{E}+01$ & $1.73 \mathrm{E}-01$ & $4.76 \mathrm{E}-01$ & $2.71 \mathrm{E}-04$ & $1.73 \mathrm{E}-01$ & $5.37 \mathrm{E}-02$ \\
\hline 18 & $4.26 \mathrm{E}+01$ & $1.01 \mathrm{E}+00$ & $3.71 \mathrm{E}+01$ & $2.36 \mathrm{E}-01$ & $1.49 \mathrm{E}+00$ & $9.44 \mathrm{E}-04$ & $1.44 \mathrm{E}+00$ & $1.07 \mathrm{E}+00$ \\
\hline 19 & $2.54 \mathrm{E}+01$ & $1.09 \mathrm{E}+00$ & $3.96 \mathrm{E}+01$ & $2.86 \mathrm{E}-01$ & $2.11 \mathrm{E}+00$ & $1.76 \mathrm{E}-03$ & $1.74 \mathrm{E}+00$ & $1.21 \mathrm{E}+00$ \\
\hline 20 & $1.89 \mathrm{E}+01$ & $1.14 \mathrm{E}+00$ & $3.89 \mathrm{E}+01$ & $3.28 \mathrm{E}-01$ & $2.53 \mathrm{E}+00$ & $2.66 \mathrm{E}-03$ & $1.98 \mathrm{E}+00$ & $1.32 \mathrm{E}+00$ \\
\hline 21 & $1.63 \mathrm{E}+01$ & $1.17 \mathrm{E}+00$ & $3.65 \mathrm{E}+01$ & $3.62 \mathrm{E}-01$ & $2.82 \mathrm{E}+00$ & $3.64 \mathrm{E}-03$ & $2.17 \mathrm{E}+00$ & $1.41 \mathrm{E}+00$ \\
\hline 22 & $1.52 \mathrm{E}+01$ & $1.20 \mathrm{E}+00$ & $3.33 \mathrm{E}+01$ & $3.90 \mathrm{E}-01$ & $3.04 \mathrm{E}+00$ & $4.66 \mathrm{E}-03$ & $2.32 \mathrm{E}+00$ & $1.48 \mathrm{E}+00$ \\
\hline 23 & $1.47 \mathrm{E}+01$ & $1.23 \mathrm{E}+00$ & $2.99 \mathrm{E}+01$ & $4.15 \mathrm{E}-01$ & $3.23 \mathrm{E}+00$ & $5.72 \mathrm{E}-03$ & $2.46 \mathrm{E}+00$ & $1.54 \mathrm{E}+00$ \\
\hline 24 & $1.44 \mathrm{E}+01$ & $1.26 \mathrm{E}+00$ & $2.66 \mathrm{E}+01$ & $4.35 \mathrm{E}-01$ & $3.39 \mathrm{E}+00$ & $6.81 \mathrm{E}-03$ & $2.58 \mathrm{E}+00$ & $1.59 \mathrm{E}+00$ \\
\hline 25 & $1.43 \mathrm{E}+01$ & $1.29 \mathrm{E}+00$ & $2.36 \mathrm{E}+01$ & $4.54 \mathrm{E}-01$ & $3.53 \mathrm{E}+00$ & 7.94E-03 & $2.68 \mathrm{E}+00$ & $1.64 \mathrm{E}+00$ \\
\hline 26 & $1.43 \mathrm{E}+01$ & $1.31 \mathrm{E}+00$ & $2.10 \mathrm{E}+01$ & $4.70 \mathrm{E}-01$ & $3.67 \mathrm{E}+00$ & $9.10 \mathrm{E}-03$ & $2.78 E+00$ & $1.69 \mathrm{E}+00$ \\
\hline 27 & $1.43 \mathrm{E}+01$ & $1.34 \mathrm{E}+00$ & $1.87 \mathrm{E}+01$ & $4.85 \mathrm{E}-01$ & $3.80 \mathrm{E}+00$ & $1.03 \mathrm{E}-02$ & $2.87 \mathrm{E}+00$ & $1.73 \mathrm{E}+00$ \\
\hline 28 & $1.43 \mathrm{E}+01$ & $1.36 \mathrm{E}+00$ & $1.69 \mathrm{E}+01$ & $4.98 \mathrm{E}-01$ & $3.93 \mathrm{E}+00$ & $1.15 \mathrm{E}-02$ & $2.96 \mathrm{E}+00$ & $1.77 \mathrm{E}+00$ \\
\hline 29 & $1.43 \mathrm{E}+01$ & $1.39 \mathrm{E}+00$ & $1.53 \mathrm{E}+01$ & $5.11 \mathrm{E}-01$ & $4.06 \mathrm{E}+00$ & $1.28 \mathrm{E}-02$ & $3.04 \mathrm{E}+00$ & $1.80 \mathrm{E}+00$ \\
\hline 30 & $1.44 \mathrm{E}+01$ & $1.41 \mathrm{E}+00$ & $1.41 \mathrm{E}+01$ & $5.24 \mathrm{E}-01$ & $4.03 E+00$ & $1.32 \mathrm{E}-02$ & $3.91 \mathrm{E}+00$ & $1.22 \mathrm{E}+00$ \\
\hline 31 & $1.45 \mathrm{E}+01$ & $1.44 \mathrm{E}+00$ & $1.31 \mathrm{E}+01$ & $5.36 \mathrm{E}-01$ & $4.01 \mathrm{E}+00$ & $1.36 \mathrm{E}-02$ & $3.90 \mathrm{E}+00$ & $1.22 \mathrm{E}+00$ \\
\hline 32 & $1.45 \mathrm{E}+01$ & $1.47 \mathrm{E}+00$ & $1.24 \mathrm{E}+01$ & $5.47 \mathrm{E}-01$ & $3.98 \mathrm{E}+00$ & $1.40 \mathrm{E}-02$ & $3.89 \mathrm{E}+00$ & $1.21 \mathrm{E}+00$ \\
\hline 33 & $1.46 \mathrm{E}+01$ & $1.49 \mathrm{E}+00$ & $1.18 \mathrm{E}+01$ & $5.59 \mathrm{E}-01$ & $3.96 \mathrm{E}+00$ & $1.44 \mathrm{E}-02$ & $3.87 \mathrm{E}+00$ & $1.21 \mathrm{E}+00$ \\
\hline 34 & $1.47 \mathrm{E}+01$ & $1.52 \mathrm{E}+00$ & $1.14 \mathrm{E}+01$ & $5.70 \mathrm{E}-01$ & $3.93 \mathrm{E}+00$ & $1.49 \mathrm{E}-02$ & $3.86 \mathrm{E}+00$ & $1.21 \mathrm{E}+00$ \\
\hline 35 & $1.48 \mathrm{E}+01$ & $1.55 \mathrm{E}+00$ & $1.11 \mathrm{E}+01$ & $5.82 \mathrm{E}-01$ & $3.91 E+00$ & $1.53 \mathrm{E}-02$ & $3.85 \mathrm{E}+00$ & $1.20 \mathrm{E}+00$ \\
\hline 36 & $1.48 \mathrm{E}+01$ & $1.57 \mathrm{E}+00$ & $1.09 \mathrm{E}+01$ & $5.93 \mathrm{E}-01$ & $3.88 \mathrm{E}+00$ & $1.57 \mathrm{E}-02$ & $3.84 \mathrm{E}+00$ & $1.20 \mathrm{E}+00$ \\
\hline 37 & $1.49 \mathrm{E}+01$ & $1.60 \mathrm{E}+00$ & $1.07 \mathrm{E}+01$ & $6.05 \mathrm{E}-01$ & $3.86 \mathrm{E}+00$ & $1.61 \mathrm{E}-02$ & $3.82 \mathrm{E}+00$ & $1.19 \mathrm{E}+00$ \\
\hline 38 & $1.49 \mathrm{E}+01$ & $1.63 \mathrm{E}+00$ & $1.07 \mathrm{E}+01$ & $6.17 \mathrm{E}-01$ & $3.84 \mathrm{E}+00$ & $1.65 \mathrm{E}-02$ & $3.81 \mathrm{E}+00$ & $1.19 \mathrm{E}+00$ \\
\hline
\end{tabular}




\begin{tabular}{|c|c|c|c|c|c|c|c|c|}
\hline 39 & $1.50 \mathrm{E}+01$ & $1.65 \mathrm{E}+00$ & $1.07 \mathrm{E}+01$ & $6.29 \mathrm{E}-01$ & $3.81 \mathrm{E}+00$ & $1.69 \mathrm{E}-02$ & $3.80 \mathrm{E}+00$ & $19 \mathrm{E}+$ \\
\hline 40 & $1.50 \mathrm{E}+01$ & $1.68 \mathrm{E}+00$ & $1.07 \mathrm{E}+01$ & $6.41 \mathrm{E}-01$ & $3.79 \mathrm{E}+00$ & $1.73 \mathrm{E}-02$ & $3.79 \mathrm{E}+00$ & $1.18 \mathrm{E}+$ \\
\hline$\overline{41}$ & $1.50 \mathrm{E}+01$ & $1.71 \mathrm{E}+00$ & $1.08 \mathrm{E}+01$ & $6.53 \mathrm{E}-01$ & $3.77 \mathrm{E}+00$ & $1.77 E-02$ & $3.78 \mathrm{E}+00$ & $.18 \mathrm{E}+$ \\
\hline 42 & $1.50 \mathrm{E}+01$ & $1.73 \mathrm{E}+00$ & $1.09 \mathrm{E}+01$ & $6.65 \mathrm{E}-01$ & $3.90 \mathrm{E}+00$ & $1.91 \mathrm{E}-02$ & $3.78 \mathrm{E}+00$ & $2.44 \mathrm{E}+$ \\
\hline 43 & $1.51 \mathrm{E}+01$ & $1.76 \mathrm{E}+00$ & $1.10 \mathrm{E}+01$ & $6.78 \mathrm{E}-01$ & $4.03 \mathrm{E}+00$ & $2.06 \mathrm{E}-02$ & $3.84 \mathrm{E}+00$ & $2.47 \mathrm{E}+$ \\
\hline 44 & $1.51 \mathrm{E}+01$ & $1.79 \mathrm{E}+00$ & $1.11 \mathrm{E}+01$ & $6.91 \mathrm{E}-01$ & $4.16 \mathrm{E}+00$ & $2.21 \mathrm{E}-02$ & $3.91 \mathrm{E}+00$ & $2.49 \mathrm{E}+$ \\
\hline 45 & $1.51 \mathrm{E}+01$ & $1.81 \mathrm{E}+00$ & $1.12 \mathrm{E}+01$ & $7.03 \mathrm{E}-01$ & $4.29 \mathrm{E}+00$ & $2.36 \mathrm{E}-02$ & $3.97 \mathrm{E}+00$ & $2.52 \mathrm{E}+0$ \\
\hline 46 & $1.51 \mathrm{E}+01$ & $1.84 \mathrm{E}+00$ & $1.13 \mathrm{E}+01$ & $7.16 \mathrm{E}-01$ & $4.42 \mathrm{E}+00$ & $2.52 \mathrm{E}-02$ & $4.03 E+00$ & $2.55 \mathrm{E}+$ \\
\hline 47 & $1.51 \mathrm{E}+01$ & $1.87 \mathrm{E}+00$ & $1.14 \mathrm{E}+01$ & $7.29 \mathrm{E}-01$ & $4.55 \mathrm{E}+00$ & $2.67 \mathrm{E}-02$ & $4.09 \mathrm{E}+00$ & $2.58 \mathrm{E}+$ \\
\hline 48 & $1.51 \mathrm{E}+01$ & $1.89 \mathrm{E}+00$ & $1.15 \mathrm{E}+01$ & $7.42 \mathrm{E}-01$ & $4.67 \mathrm{E}+00$ & $2.83 \mathrm{E}-02$ & $4.16 \mathrm{E}+00$ & $2.61 \mathrm{E}+$ \\
\hline 49 & $1.51 \mathrm{E}+01$ & $1.92 \mathrm{E}+00$ & $1.15 \mathrm{E}+01$ & $7.55 \mathrm{E}-01$ & $4.80 \mathrm{E}+00$ & $3.00 \mathrm{E}-02$ & $4.22 \mathrm{E}+00$ & $2.64 \mathrm{E}+$ \\
\hline 50 & $1.51 \mathrm{E}+01$ & $1.95 \mathrm{E}+00$ & $1.16 \mathrm{E}+01$ & $7.68 \mathrm{E}-01$ & $4.92 \mathrm{E}+00$ & $3.16 \mathrm{E}-02$ & $4.28 \mathrm{E}+00$ & $2.67 \mathrm{E}+\mathrm{C}$ \\
\hline 51 & $1.50 \mathrm{E}+01$ & $1.97 \mathrm{E}+00$ & $1.16 \mathrm{E}+01$ & $7.81 \mathrm{E}-01$ & $5.05 \mathrm{E}+00$ & $3.33 \mathrm{E}-02$ & $4.34 \mathrm{E}+00$ & $2.69 \mathrm{E}-1$ \\
\hline 52 & $1.50 \mathrm{E}+01$ & $2.00 \mathrm{E}+00$ & $1.16 \mathrm{E}+01$ & $7.94 \mathrm{E}-01$ & $5.17 \mathrm{E}+00$ & $3.51 \mathrm{E}-02$ & $4.40 \mathrm{E}+00$ & $2.72 \mathrm{E}+$ \\
\hline 53 & $1.50 \mathrm{E}+01$ & $2.03 \mathrm{E}+00$ & $1.16 \mathrm{E}+01$ & $8.07 \mathrm{E}-01$ & $5.29 \mathrm{E}+00$ & $3.68 \mathrm{E}-02$ & $4.46 \mathrm{E}+00$ & $2.75 \mathrm{E}+\mathrm{C}$ \\
\hline 54 & $1.49 \mathrm{E}+01$ & $2.05 \mathrm{E}+00$ & $1.17 \mathrm{E}+01$ & $8.20 \mathrm{E}-01$ & $5.25 \mathrm{E}+00$ & $3.73 \mathrm{E}-02$ & $5.09 \mathrm{E}+00$ & $1.59 \mathrm{E}+$ \\
\hline 55 & $1.48 \mathrm{E}+01$ & $2.08 \mathrm{E}+00$ & $1.17 \mathrm{E}+01$ & $8.33 \mathrm{E}-01$ & $5.21 \mathrm{E}+00$ & $3.79 \mathrm{E}-02$ & $5.07 \mathrm{E}+00$ & $1.59 \mathrm{E}+$ \\
\hline 56 & $1.47 \mathrm{E}+01$ & $2.10 \mathrm{E}+00$ & $1.17 \mathrm{E}+01$ & $8.46 \mathrm{E}-01$ & $5.18 \mathrm{E}+00$ & $3.84 \mathrm{E}-02$ & $5.05 \mathrm{E}+00$ & $1.58 \mathrm{E}+\mathrm{C}$ \\
\hline 57 & $1.46 \mathrm{E}+01$ & $2.13 \mathrm{E}+00$ & $1.16 \mathrm{E}+01$ & $8.59 \mathrm{E}-0 \mathrm{1}$ & $5.14 \mathrm{E}+00$ & $3.89 \mathrm{E}-02$ & $5.04 \mathrm{E}+00$ & $1.58 \mathrm{E}+$ \\
\hline 58 & $1.45 \mathrm{E}+01$ & $2.16 \mathrm{E}+00$ & $1.16 \mathrm{E}+01$ & $8.72 \mathrm{E}-01$ & $5.11 \mathrm{E}+00$ & $3.95 \mathrm{E}-02$ & $5.02 \mathrm{E}+00$ & $1.57 \mathrm{E}+$ \\
\hline 59 & $1.44 \mathrm{E}+01$ & $2.18 \mathrm{E}+00$ & $1.16 \mathrm{E}+01$ & $8.84 \mathrm{E}-01$ & $5.07 \mathrm{E}+00$ & 4.00E-02 & $5.00 \mathrm{E}+00$ & $1.57 \mathrm{E}+\mathrm{C}$ \\
\hline 60 & $1.43 \mathrm{E}+01$ & $2.21 \mathrm{E}+00$ & $1.15 \mathrm{E}+01$ & $8.97 \mathrm{E}-01$ & $5.04 E+00$ & $4.05 \mathrm{E}-02$ & $4.99 \mathrm{E}+00$ & $1.56 \mathrm{E}+0$ \\
\hline 61 & $1.42 \mathrm{E}+01$ & $2.23 \mathrm{E}+00$ & $1.15 \mathrm{E}+01$ & $9.10 \mathrm{E}-01$ & $5.01 E+00$ & $4.10 \mathrm{E}-02$ & $4.97 \mathrm{E}+00$ & $1.56 \mathrm{E}+\mathrm{C}$ \\
\hline 62 & $1.41 \mathrm{E}+01$ & $2.25 \mathrm{E}+00$ & $1.14 \mathrm{E}+01$ & $9.22 \mathrm{E}-01$ & $4.97 \mathrm{E}+00$ & $4.15 E-02$ & $4.95 \mathrm{E}+00$ & $1.55 \mathrm{E}+\mathrm{C}$ \\
\hline 63 & $1.39 \mathrm{E}+01$ & $2.28 \mathrm{E}+00$ & $1.14 \mathrm{E}+01$ & $9.35 \mathrm{E}-01$ & $4.94 \mathrm{E}+00$ & $4.20 \mathrm{E}-02$ & $4.94 \mathrm{E}+00$ & $1.54 \mathrm{E}+\mathrm{C}$ \\
\hline 64 & $1.38 \mathrm{E}+01$ & $2.30 \mathrm{E}+00$ & $1.13 \mathrm{E}+01$ & $9.47 \mathrm{E}-01$ & $4.90 \mathrm{E}+00$ & $4.25 \mathrm{E}-02$ & $4.92 \mathrm{E}+00$ & $1.54 \mathrm{E}+\mathrm{C}$ \\
\hline 65 & $1.36 \mathrm{E}+01$ & $2.33 \mathrm{E}+00$ & $1.12 \mathrm{E}+01$ & $9.59 \mathrm{E}-01$ & $4.87 \mathrm{E}+00$ & $4.30 \mathrm{E}-02$ & $4.90 \mathrm{E}+00$ & $1.53 \mathrm{E}+\mathrm{C}$ \\
\hline 66 & $1.34 \mathrm{E}+01$ & $2.35 \mathrm{E}+00$ & $1.11 \mathrm{E}+01$ & $9.71 \mathrm{E}-01$ & $4.98 \mathrm{E}+00$ & $4.49 \mathrm{E}-02$ & $4.82 \mathrm{E}+00$ & $3.21 \mathrm{E}+0$ \\
\hline 67 & $1.33 \mathrm{E}+01$ & $2.37 \mathrm{E}+00$ & $1.10 \mathrm{E}+01$ & $9.83 \mathrm{E}-01$ & $5.08 \mathrm{E}+00$ & $4.68 \mathrm{E}-02$ & $4.88 \mathrm{E}+00$ & $3.23 \mathrm{E}+0$ \\
\hline 68 & $1.31 \mathrm{E}+01$ & $2.40 \mathrm{E}+00$ & $1.09 \mathrm{E}+01$ & $9.95 \mathrm{E}-01$ & $5.19 \mathrm{E}+00$ & $4.88 \mathrm{E}-02$ & $4.93 \mathrm{E}+00$ & $3.26 \mathrm{E}+\mathrm{C}$ \\
\hline 69 & $1.29 \mathrm{E}+01$ & $2.42 \mathrm{E}+00$ & $1.08 \mathrm{E}+01$ & $1.01 \mathrm{E}+00$ & $5.29 \mathrm{E}+00$ & $5.07 \mathrm{E}-02$ & $4.98 \mathrm{E}+00$ & $3.28 \mathrm{E}+0$ \\
\hline 168 & $1.31 \mathrm{E}+00$ & $3.42 \mathrm{E}+00$ & $2.41 \mathrm{E}+00$ & $1.66 \mathrm{E}+00$ & $5.36 \mathrm{E}+00$ & $2.16 \mathrm{E}-01$ & $5.52 \mathrm{E}+00$ & $4.13 \mathrm{E}+0$ \\
\hline 336 & $5.28 \mathrm{E}-01$ & $2.84 \mathrm{E}+00$ & $5.72 \mathrm{E}-01$ & $1.75 \mathrm{E}+00$ & $2.10 \mathrm{E}+00$ & $5.17 \mathrm{E}-01$ & $3.36 \mathrm{E}+00$ & $3.18 \mathrm{E}+0$ \\
\hline 504 & $3.77 \mathrm{E}-01$ & $2.33 \mathrm{E}+00$ & $3.81 \mathrm{E}-01$ & $1.80 \mathrm{E}+00$ & $9.89 \mathrm{E}-01$ & $6.83 \mathrm{E}-01$ & $1.41 \mathrm{E}+00$ & $2.10 \mathrm{E}+0$ \\
\hline 720 & $2.72 \mathrm{E}-01$ & $1.80 \mathrm{E}+00$ & $2.52 \mathrm{E}-01$ & $1.84 \mathrm{E}+00$ & $4.95 \mathrm{E}-01$ & $7.77 E-01$ & $6.81 \mathrm{E}-01$ & $1.45 \mathrm{E}+0$ \\
\hline 2160 & $5.01 \mathrm{E}-02$ & $3.19 \mathrm{E}-01$ & $2.79 \mathrm{E}-02$ & $1.92 \mathrm{E}+00$ & $4.23 \mathrm{E}-02$ & $4.70 \mathrm{E}-01$ & $1.56 \mathrm{E}-01$ & $4.88 \mathrm{E}-0$ \\
\hline 4320 & $4.81 \mathrm{E}-03$ & $2.50 \mathrm{E}-02$ & $1.41 \mathrm{E}-01$ & $1.84 \mathrm{E}+00$ & $6.15 \mathrm{E}-03$ & $1.11 \mathrm{E}-01$ & $1.70 \mathrm{E}-02$ & $7.22 \mathrm{E}-0$ \\
\hline 4800 & $2.86 \mathrm{E}-03$ & $1.43 \mathrm{E}-02$ & $1.41 \mathrm{E}-01$ & $1.84 \mathrm{E}+00$ & $4.40 \mathrm{E}-03$ & $7.94 \mathrm{E}-02$ & $5.22 \mathrm{E}-03$ & $2.28 \mathrm{E}-0$ \\
\hline 8760 & $3.93 \mathrm{E}-05$ & $1.61 \mathrm{E}-04$ & $1.37 \mathrm{E}-01$ & $1.79 \mathrm{E}+00$ & $3.30 \mathrm{E}-04$ & $5.06 \mathrm{E}-03$ & $1.57 \mathrm{E}-03$ & $6.55 \mathrm{E}-0$ \\
\hline $3 \mathbf{T}$ & $\begin{array}{l}2.4 \mathrm{E} 6 \\
\mathrm{pCi} / \mathrm{L}\end{array}$ & $\begin{array}{l}8.88 \mathrm{E}+4 \\
\mathrm{~Bq} / \mathrm{kg}\end{array}$ & $.8 \mathrm{~Bq} / \mathrm{g}$ & & & & & \\
\hline TO & $\begin{array}{l}5.9 \mathrm{E} 6 \\
\mathrm{pCi} / \mathrm{L} \\
\end{array}$ & $\begin{array}{l}2.18 \mathrm{E}+5 \\
\mathrm{~Bq} / \mathrm{kg}\end{array}$ & $218.3 \mathrm{~Bq} / \mathrm{g}$ & & & & & \\
\hline & \multicolumn{2}{|c|}{ VEGETABLES } & \multicolumn{2}{|c|}{ WHEAT SEED } & \multicolumn{2}{|l|}{ MEAT } & \multicolumn{2}{|l|}{ MILK } \\
\hline \multirow[t]{2}{*}{ HOUR } & HTO & OBT & HTO & $\mathrm{OBT}$ & HTO & DBT & HTO & OBT \\
\hline & $(\mathrm{Bq} / \mathrm{g})$ & $(\overline{B q} / \mathrm{g})$ & $(\mathrm{Bq} / \mathrm{g})$ & $(\mathrm{Bq} / \mathrm{g})$ & $(\mathrm{Bq} / \mathrm{g})$ & $(\mathrm{Bq} / \mathrm{g})$ & $(\mathrm{Bq} / \mathrm{g})$ & $(\mathrm{Bq} / \mathrm{g})$ \\
\hline ak HTO & & & & & & & & \\
\hline
\end{tabular}




\begin{tabular}{|c|c|c|c|c|c|c|c|c|}
\hline $\begin{array}{l}17 / 19 / 29 / 5 \\
4\end{array}$ & $9.01 \mathrm{E}+01$ & 8.37E-01 & $3.96 \mathrm{E}+01$ & $2.86 \mathrm{E}-01$ & $4.06 \mathrm{E}+00$ & $1.28 \mathrm{E}-02$ & $5.09 \mathrm{E}+00$ & $1.59 \mathrm{E}+00$ \\
\hline$\overline{\text { SUM }}$ & $9.09 E+01$ & & $3.99 \mathrm{E}+01$ & & $4.07 E+00$ & & $6.68 \mathrm{E}+00$ & \\
\hline $\mathrm{Ci} / \mathrm{g}$ & $2.46 \mathrm{E}-09$ & & $1.08 \mathrm{E}-09$ & & $1.10 \mathrm{E}-10$ & & $1.81 \mathrm{E}-10$ & \\
\hline & & & & & & & & \\
\hline DIL HTO & $5.9 \mathrm{E}-09$ & & & & & & & \\
\hline \multicolumn{9}{|l|}{$(\mathrm{Ci} / \mathrm{g})$} \\
\hline & & & & & & & & \\
\hline 168 & $1.31 \mathrm{E}+00$ & $3.42 \mathrm{E}+00$ & $2.41 E+00$ & $1.66 \mathrm{E}+00$ & $5.36 \mathrm{E}+00$ & $2.16 \mathrm{E}-01$ & $5.52 \mathrm{E}+00$ & $4.13 E+00$ \\
\hline SUM & $4.74 \mathrm{E}+00$ & & $4.07 \mathrm{E}+00$ & & $5.57 \mathrm{E}+00$ & & $9.65 \bar{E}+00$ & \\
\hline$\overline{\mathrm{Ci} / \mathrm{g}}$ & $1.28 \mathrm{E}-10$ & & $1.10 \mathrm{E}-10$ & & $1.51 \mathrm{E}-10$ & & $2.61 \mathrm{E}-10$ & \\
\hline DIL OBT & $2.4 \mathrm{E}-09$ & 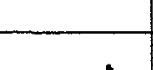 & & & & & & \\
\hline \multicolumn{9}{|l|}{$\mathrm{Ci} / \mathrm{g}$} \\
\hline & & & & & & & & \\
\hline 8760 & $3.93 \mathrm{E}-05$ & $1.61 \mathrm{E}-04$ & $1.37 \mathrm{E}-01$ & $1.79 E+00$ & $3.30 \mathrm{E}-04$ & $5.06 \mathrm{E}-03$ & $1.57 \mathrm{E}-03$ & $6.55 \mathrm{E}-03$ \\
\hline SUM & $2.00 \mathrm{E}-04$ & & $1.93 \mathrm{E}+00$ & & $5.38 E-03$ & & $8.12 \mathrm{E}-03$ & \\
\hline $\mathrm{Ci} / \mathrm{g}$ & $5.42 \mathrm{E}-15$ & & $5.21 \mathrm{E}-11$ & & $1.46 \mathrm{E}-13$ & & $2.19 \mathrm{E}-13$ & \\
\hline & & & & & & & & \\
\hline DIL OBT & $2.4 \mathrm{E}-09$ & & & & & & & \\
\hline $\mathrm{Ci} / \mathrm{g}$ & & & & & & & & \\
\hline & & & & & & & & \\
\hline
\end{tabular}

